ESAIM: COCV 27 (2021) 75

https://doi.org/10.1051/cocv/2021068
ESAIM: Control, Optimisation and Calculus of Variations

www.esaim-cocv.org

\title{
TRANSFORMATION PRESERVING CONTROLLABILITY FOR NONLINEAR OPTIMAL CONTROL PROBLEMS WITH JOINT BOUNDARY CONDITIONS*
}

\author{
ROMAN ŠImon HILSCHER ${ }^{1, * *}$ ANd Vera M. ZeIdAN ${ }^{2}$
}

\begin{abstract}
In this paper we develop a new approach for optimal control problems with general jointly varying state endpoints (also called coupled endpoints). We present a new transformation of a nonlinear optimal control problem with jointly varying state endpoints and pointwise equality control constraints into an equivalent optimal control problem of the same type but with separately varying state endpoints in double dimension. Our new transformation preserves among other properties the controllability (normality) of the considered optimal control problems. At the same time it is well suited even for the calculus of variations problems with joint state endpoints, as well as for optimal control problems with free initial and/or final time. This work is motivated by the results on the second order Sturm-Liouville eigenvalue problems with joint endpoints by Dwyer and Zettl (1994) and by the sensitivity result for nonlinear optimal control problems with separated state endpoints by the authors (2018).
\end{abstract}

Mathematics Subject Classification. 49K15, 49K40, 90C31.

Received July 31, 2020. Accepted June 22, 2021.

\section{INTRODUCTION}

In this paper we develop a new approach to optimal control problems with general jointly varying state endpoints (also called coupled endpoints), including the special case with periodic or antiperiodic endpoints. Necessary or sufficient optimality conditions for such optimal control problems are usually derived by a direct analysis of the problem, in particular when dealing with the nonnegativity or coercivity of the second variation, the Jacobi system and the Riccati differential equation, or the Hamilton-Jacobi theory, see e.g. $[1,2,4,5,19$, 26-29, 35, 36].

In the literature one can also find an alternative approach based on transforming the problem with joint endpoints into an augmented problem with separated endpoints in double dimension. This method has been used in particular in the context of the Jacobi system (or the linear Hamiltonian system) and the corresponding Riccati equation, see e.g. [1, 14-16, 20,36], and it consists of doubling the dimension of the original state

\footnotetext{
* This research was supported by the Czech Science Foundation under grant GA19-01246S.

Keywords and phrases: Optimal control problem, joint (coupled) endpoints, separated endpoints, controllability, strong Pontryagin principle, coercivity, sensitivity analysis, free time problem.

${ }^{1}$ Department of Mathematics and Statistics, Faculty of Science, Masaryk University, Kotlářská 2, 61137 Brno, Czech Republic.

2 Department of Mathematics, Michigan State University, East Lansing, MI 48824-1027, USA.

** Corresponding author: hilscher@math.muni.cz
} 
and adjoint variables by suitable constant quantities. The utility of this method has been revived due to the development during the last two decades of the theory of linear Hamiltonian and symplectic systems without the controllability (normality) assumption. For instance, important results in the continuous and discrete oscillation and spectral theory for problems with joint endpoints were derived by means of the above transformation to the separated endpoints setting in double dimension, see e.g. [3, 10, 12, 13, 15, 16, 30, 32, 34].

On the other hand, for the nonlinear optimal control problems, meaningful second order necessary optimality conditions, or sufficient optimality conditions for non-isolated candidates are derived in the literature under a certain controllability (normality) assumption imposed on the linearized system, see e.g. [17, 22, 23, 25$27,33,36,39,40]$. In page 886 of [36], the transformation described above was suggested as a tool to extend to the joint endpoints case the results on optimality conditions established therein for the separated endpoints problem and involving the second variation phrased in terms of the associated linear Hamiltonian system and the corresponding Riccati differential equation. The extended Riccati type sufficient condition for nonlinear periodic optimal control problems from Theorem 2.1 of [1] is an example of the above approach, although the authors prove their result directly without an explicit usage of this transformation. Note that the latter condition is a special case of the solvability condition for the augmented Riccati differential equation presented in Theorem 6.2(iii) of [14].

In this paper we present a new transformation of a general nonlinear optimal control problem with jointly varying state endpoints and pointwise equality control constraints into an equivalent optimal control problem of the same type but with separately varying state endpoints in double dimension (see the results in Sect. 3). The advantage of our new transformation is that it preserves the controllability (normality) of the considered optimal control problems, as well as the normality of the associated Jacobi systems. This transformation is suitable and new even for the calculus of variations problems with jointly varying endpoints, as it preserves the structure of this type of problem. This work is motivated by the results on the second order Sturm-Liouville eigenvalue problems with joint endpoints in $[8,9]$ by Dwyer and Zettl. The differential equation considered in [9] represents the Jacobi equation (or the Euler-Lagrange equation) for a calculus of variations problem. There it is suggested to flip the interval $[a, b]$ over its midpoint

$$
c:=(a+b) / 2
$$

and to consider an augmented eigenvalue problem with separated endpoints in double dimension over the halfinterval $[a, c]$ or $[c, b]$. In this paper we extend this method to the general setting of nonlinear optimal control problems. The new transformation method allows, for example, to extend the sensitivity results for optimal control problems with separated state endpoints in Theorem 4.3 of [33] to arbitrary jointly varying endpoints (Thm. 4.1). Note that the result in [33] is an extension to separable endpoints of the fixed initial state constraints in $[22,24,25]$. In other words, we demonstrate that this new transformation is a useful tool that serves to extend to jointly varying endpoints the results known for separated endpoints. This transformation is also well-suited for optimal control problems with free initial and/or final time, as it allows to separate the initial free time and state constraints from the final free time and state constraints.

The paper is organized as follows. In Section 2 we introduce the optimal control problem (C) on the interval $[a, b]$ with joint state endpoints, including the notion of normality, the strong and weak Pontryagin principles, and the second variation. In Section 3 we develop the transformation of this problem into an equivalent augmented optimal control problem $(\tilde{\mathrm{C}})$ with separated state endpoints. In Section 4 we illustrate the utility of this method by deriving a new sensitivity result for optimal control problems with jointly varying state endpoints. In Section 5 we discuss the transformation in the context of optimal control problems with free initial and/or final time. It is worth mentioning that the transformation developed in this paper is not restricted to the spaces of controls and states considered here, but it applies to any other spaces, e.g., to $L^{\infty}$ controls and absolutely continuous states. 


\section{Optimal CONTROL PROBLEM WITH JOINT STATE ENDPOINTS}

Consider the nonlinear optimal control problem over the interval $[a, b]$ with jointly varying state endpoints

$$
\text { minimize } J(x, u):=K(x(a), x(b))+\int_{a}^{b} L(t, x(t), u(t)) \mathrm{d} t
$$

subject to $x(\cdot) \in \mathrm{C}_{\mathrm{p}}^{1}\left([a, b], \mathbb{R}^{n}\right)$ and $u(\cdot) \in \mathrm{C}_{\mathrm{p}}\left([a, b], \mathbb{R}^{m}\right)$ such that

$$
\begin{gathered}
\dot{x}(t)=f(t, x(t), u(t)), \quad t \in[a, b], \\
\psi(t, u(t))=0, \quad t \in[a, b], \\
\varphi(x(a), x(b))=0 .
\end{gathered}
$$

We use the notation $\mathrm{C}_{\mathrm{p}}^{1}(\mathcal{I}, V)$ and $\mathrm{C}_{\mathrm{p}}(\mathcal{I}, V)$ for the spaces of piecewise continuously differentiable functions and piecewise continuous functions on the interval $\mathcal{I}$ with values in the target space $V$. We assume that $n, m, k, r \in \mathbb{N}$ are given dimensions with $k \leq m$ and $r \leq 2 n$ and

$$
\begin{aligned}
& L:[a, b] \times \mathbb{R}^{n} \times \mathbb{R}^{m} \rightarrow \mathbb{R}, \quad K: \mathbb{R}^{n} \times \mathbb{R}^{n} \rightarrow \mathbb{R}, \\
& f:[a, b] \times \mathbb{R}^{n} \times \mathbb{R}^{m} \rightarrow \mathbb{R}^{n}, \quad \varphi: \mathbb{R}^{n} \times \mathbb{R}^{n} \rightarrow \mathbb{R}^{r}, \\
& \psi:[a, b] \times \mathbb{R}^{m} \rightarrow \mathbb{R}^{k} .
\end{aligned}
$$

Given that $u(\cdot) \in \mathrm{C}_{\mathrm{p}}\left([a, b], \mathbb{R}^{m}\right)$, we require that equations (2.1) and (2.2) are satisfied at the left and right limits of its discontinuity points. The Hamiltonian

$$
H:[a, b] \times \mathbb{R}^{n} \times \mathbb{R}^{m} \times \mathbb{R}^{n} \times \mathbb{R}^{k} \times \mathbb{R} \rightarrow \mathbb{R}
$$

corresponding to problem $(\mathrm{C})$ is defined by

$$
H\left(t, x, u, p, \lambda, \lambda_{0}\right):=p^{T} f(t, x, u)+\lambda_{0} L(t, x, u)+\lambda^{T} \psi(t, u) .
$$

A pair $(x(\cdot), u(\cdot))$ is feasible for problem $(\mathrm{C})$ if $x(\cdot) \in \mathrm{C}_{\mathrm{p}}^{1}\left([a, b], \mathbb{R}^{n}\right)$ and $u(\cdot) \in \mathrm{C}_{\mathrm{p}}\left([a, b], \mathbb{R}^{m}\right)$ and it satisfies equations $(2.1)-(2.3)$. A feasible pair $(\hat{x}(\cdot), \hat{u}(\cdot))$ is a strong local minimum, respectively, a weak local minimum, for problem (C) if there exists $\delta>0$ such that we have $J(\hat{x}, \hat{u}) \leq J(x, u)$ for any feasible pair $(x(\cdot), u(\cdot))$ with $\|x(\cdot)-\hat{x}(\cdot)\|_{\mathrm{C}_{\mathrm{p}}[a, b]}<\delta$, respectively, with $\|(x(\cdot)-\hat{x}(\cdot), u(\cdot)-\hat{u}(\cdot))\|_{\mathrm{C}_{\mathrm{p}}[a, b]}<\delta$, where

$$
\|z(\cdot)\|_{\mathrm{C}_{\mathrm{p}}[a, b]}:=\sup _{t \in[a, b]}|z(t)| .
$$

If the inequality $J(\hat{x}, \hat{u})<J(x, u)$ holds for all such feasible pairs $(x(\cdot), u(\cdot)) \neq(\hat{x}(\cdot), \hat{u}(\cdot))$, then $(\hat{x}(\cdot), \hat{u}(\cdot))$ is a strict strong local minimum, respectively, a strict weak local minimum for problem (C). It is clear that if a pair $(\hat{x}(\cdot), \hat{u}(\cdot))$ is a (strict) strong local minimum for problem $(\mathrm{C})$, then it is also a (strict) weak local minimum for problem $(\mathrm{C})$.

We shall use the following definition from Section 2 of [38]. A function $h(t, \cdot, \cdot)$ is continuous at the feasible pair $(x(\cdot), u(\cdot))$ uniformly in $t$ on $[a, b]$ if for every $\varepsilon>0$ there exists $\delta>0$ such that for all $t \in[a, b]$ and all $(x, u) \in \mathbb{R}^{n} \times \mathbb{R}^{m}$ with $0<|(x, u)-(x(t), u(t))|<\delta$ we have $|h(t, x, u)-h(t, x(t), u(t))|<\varepsilon$.

In order to simplify the presentation of this paper and concentrate on the transformation of the endpoints constraints, we use the following assumptions on the regularity of the data $h(t, x, u):=(L(t, x, u), f(t, x, u), \psi(t, u))$, $K(x, y)$, and $\varphi(x, y)$ of problem (C) near a reference pair $(x(\cdot), u(\cdot))$. We wish to keep in mind that the results of this paper remain valid when these regularity assumptions are weakened, for instance, to encompass the case 
of the $L^{\infty}$-controls and absolutely continuous states. Let $(x(\cdot), u(\cdot))$ be a feasible pair and denote by $B_{\delta}(y)$ the open ball centered at $y$ of the radius $\delta$. The variable in the subscript indicates the partial derivative with respect to that variable.

(A0) There exists $\delta_{1}>0$ such that for each $(t, u)$ the function $h(t, \cdot, u)$ is differentiable on $B_{\delta_{1}}(x(t))$ and the functions $h$ and $\nabla_{x} h$ are continuous; the functions $K(\cdot, \cdot)$ and $\varphi(\cdot, \cdot)$ are continuously differentiable on $B_{\delta_{1}}(x(a), x(b))$ and the matrix $\nabla \varphi(x(a), x(b))$ has full rank.

(A1) There exists $\delta_{1}>0$ such that for each $t \in[a, b]$ the function $h(t, \cdot, \cdot)$ is differentiable on $B_{\delta_{1}}(x(t), u(t))$ and the functions $h$ and $\nabla_{(x, u)} h$ are continuous; the functions $K(\cdot, \cdot)$ and $\varphi(\cdot, \cdot)$ are continuously differentiable on $B_{\delta_{1}}(x(a), x(b))$ and the matrices $\nabla \varphi(x(a), x(b))$ and $\psi_{u}(t, u(t))$ for $t \in[a, b]$ have full rank.

(A2) There exists $\delta_{1}>0$ such that for each $t \in[a, b]$ the function $h(t, \cdot, \cdot)$ is twice differentiable on $B_{\delta_{1}}(x(t), u(t))$ and the functions $h, \nabla_{(x, u)} h$, and $\nabla_{(x, u)}^{2} h$ are continuous; the functions $K(\cdot, \cdot)$ and $\varphi(\cdot, \cdot)$ are twice continuously differentiable on $B_{\delta_{1}}(x(a), x(b))$ and the matrices $\nabla \varphi(x(a), x(b))$ and $\psi_{u}(t, u(t))$ for $t \in[a, b]$ have full rank.

Assume that (A1) holds at $(x(\cdot), u(\cdot))$. Then the linearization of constraints $(2.1)-(2.3)$ at the feasible pair $(x(\cdot), u(\cdot))$ has the form

$$
\dot{\eta}(t)=\mathcal{A}(t) \eta(t)+\mathcal{B}(t) v(t), \quad N(t) v(t)=0, \quad t \in[a, b], \quad M\left(\begin{array}{c}
\eta(a) \\
\eta(b)
\end{array}\right)=0,
$$

where $\eta(\cdot) \in \mathrm{C}_{\mathrm{p}}^{1}\left([a, b], \mathbb{R}^{n}\right)$ and $v(\cdot) \in \mathrm{C}_{\mathrm{p}}\left([a, b], \mathbb{R}^{m}\right)$ represent the linearizations of the state $x(\cdot)$ and of the control $u(\cdot)$, and where the matrix functions $\mathcal{A}(\cdot) \in \mathrm{C}_{\mathrm{p}}\left([a, b], \mathbb{R}^{n \times n}\right), \mathcal{B}(\cdot) \in \mathrm{C}_{\mathrm{p}}\left([a, b], \mathbb{R}^{n \times m}\right), N(\cdot) \in \mathrm{C}_{\mathrm{p}}\left([a, b], \mathbb{R}^{k \times m}\right)$, and $M \in \mathbb{R}^{r \times 2 n}$ are defined by

$$
\left.\begin{array}{rlrl}
\mathcal{A}(t) & :=f_{x}(t, x(t), u(t)), & \mathcal{B}(t) & :=f_{u}(t, x(t), u(t)), \\
N(t) & :=\psi_{u}(t, u(t)), & M & :=\nabla \varphi(x(a), x(b)) .
\end{array}\right\}
$$

In some cases we will split the full-rank matrix $M=\left(M_{a}, M_{b}\right)$ with $r \times n$ blocks $M_{a}$ and $M_{b}$. With the matrix function $N(\cdot)$ we associate $Y(\cdot) \in \mathrm{C}_{\mathrm{p}}\left([a, b], \mathbb{R}^{m \times(m-k)}\right)$ that denotes the matrix function for which the columns of $Y(t)$ form an orthonormal basis for the kernel of $N(t)$, i.e., $N(t) Y(t)=0$ and $Y^{T}(t) Y(t)=I_{m-k}$ on $[a, b]$.

Definition 2.1. Assume that (A0) is satisfied at $(x(\cdot), u(\cdot))$. We say that the feasible pair $(x(\cdot), u(\cdot))$ satisfies the strong Pontryagin principle for problem (C) if there exist a constant $\lambda_{0} \geq 0$, a vector $\gamma \in \mathbb{R}^{r}$, and a function $p(\cdot) \in \mathrm{C}_{\mathrm{p}}^{1}\left([a, b], \mathbb{R}^{n}\right)$ satisfying

(i) the nondegeneracy condition

$$
\lambda_{0}+\|p(\cdot)\|_{\mathrm{C}_{\mathrm{p}}[a, b]} \neq 0,
$$

(ii) the adjoint equation

$$
-\dot{p}(t)=\mathcal{A}^{T}(t) p(t)+\lambda_{0} L_{x}^{T}(t, x(t), u(t)), \quad t \in[a, b],
$$

(iii) the transversality condition

$$
\left(\begin{array}{c}
-p(a) \\
p(b)
\end{array}\right)=\lambda_{0} \nabla K^{T}(x(a), x(b))+M^{T} \gamma
$$


(iv) $)_{\mathrm{s}}$ the minimality condition

$$
\min _{u \in \mathbb{R}^{m}, \psi(t, u)=0} H\left(t, x(t), u, p(t), 0, \lambda_{0}\right) \quad \text { is attained at } u(t), t \in[a, b]
$$

where the matrices $\mathcal{A}(t)$ and $M$ are defined in (2.6).

Due to the full rank property of $M$, the nondegeneracy condition (2.7) is equivalent to the seemingly stronger condition $\lambda_{0}+\|p(\cdot)\|_{\mathrm{C}_{\mathrm{p}}[a, b]}+|\gamma| \neq 0$. Note that the subscript "s" in condition (iv) above refers to the strong Pontryagin principle, while the subscript "w" in condition (iv) w $_{\mathrm{w}}$ below refers to the weak Pontryagin principle.

Definition 2.2. Assume that (A1) holds at $(x(\cdot), u(\cdot))$. We say that the feasible pair $(x(\cdot), u(\cdot))$ satisfies the weak Pontryagin principle for problem $(\mathrm{C})$ if there exists in addition to $\lambda_{0} \geq 0, \gamma \in \mathbb{R}^{r}$, and $p(\cdot) \in \mathrm{C}_{\mathrm{p}}^{1}\left([a, b], \mathbb{R}^{n}\right)$, a function $\lambda(\cdot) \in \mathrm{C}_{\mathrm{p}}\left([a, b], \mathbb{R}^{k}\right)$ such that conditions (i)-(iii) of Definition 2.1 hold and, instead of (iv) , we have (iv) $)_{\mathrm{w}}$ the stationarity condition

$$
\mathcal{B}^{T}(t) p(t)+\lambda_{0} L_{u}^{T}(t, x(t), u(t))+N^{T}(t) \lambda(t)=0, \quad t \in[a, b]
$$

where the matrices $\mathcal{B}(t)$ and $N(t)$ are defined in (2.6).

The adjoint equation (2.8) combined with stationarity condition (2.11) can be written in terms of the Hamiltonian $H$ in $(2.4)$ as

$$
\begin{gathered}
-\dot{p}(t)=H_{x}^{T}\left(t, x(t), u(t), p(t), \lambda(t), \lambda_{0}\right), \quad t \in[a, b], \\
H_{u}^{T}\left(t, x(t), u(t), p(t), \lambda(t), \lambda_{0}\right)=0, \quad t \in[a, b] .
\end{gathered}
$$

Also, due to the full rank property of $M$ and $N(t)$ on $[a, b]$, the nondegeneracy condition (2.7) is now equivalent to $\lambda_{0}+\|p(\cdot)\|_{\mathrm{C}_{\mathrm{p}}[a, b]}+|\gamma|+\|\lambda(\cdot)\|_{\mathrm{C}_{\mathrm{p}}[a, b]} \neq 0$.

Remark 2.3. If (A1) holds at $(x(\cdot), u(\cdot))$ and if the minimality condition (2.10) of Definition 2.1 is satisfied, then the stationarity condition (2.11) holds. In fact, by the regularity of control constraints at $u(t)$ (i.e., $N(t)$ is of full rank) and by the Kuhn-Tucker condition (see e.g. [21]), there exists $\lambda(t)$ satisfying the stationarity condition (iv) $)_{\mathrm{w}}$. That this $\lambda(\cdot) \in \mathrm{C}_{\mathrm{p}}\left([a, b], \mathbb{R}^{k}\right)$ is again a result of the constraints regularity.

In the following we define the notion of normality of problem $(\mathrm{C})$.

Definition 2.4. Problem $(\mathrm{C})$ is said to be normal at a feasible pair $(x(\cdot), u(\cdot))$ satisfying the strong Pontryagin principle if there are no multipliers $\lambda_{0}=0, p(\cdot)$, and $\gamma$ associated to $(x(\cdot), u(\cdot))$ at which conditions $(2.7)$ (2.10) of Definition 2.1 hold. Similarly, problem (C) is normal at a feasible pair $(x(\cdot), u(\cdot))$ satisfying the weak Pontryagin principle if there are no multipliers $\lambda_{0}=0, p(\cdot), \lambda(\cdot)$, and $\gamma$ associated to $(x(\cdot), u(\cdot))$ at which conditions (2.7)-(2.9) and (2.11) of Definition 2.2 hold.

Remark 2.5. (i) When the normality in (C) holds for either the weak or the strong Pontryagin principle, the value of $\lambda_{0}$ could be taken to be 1 . In this case, the remaining multipliers are unique due to the full rank assumptions on $N(t)$ for all $t \in[a, b]$ and/or on $M$.

(ii) It follows from Remark 2.3 that when assumption (A1) holds, the normality of (C) with respect to the weak Pontryagin principle implies the normality of $(\mathrm{C})$ with respect to the strong Pontryagin principle.

Remark 2.6. The normality of a feasible pair $(x(\cdot), u(\cdot))$ satisfying the weak Pontryagin principle is shown in Theorem 2.1 of [41] or in Proposition 4.5 of [17] to be equivalent to the $M$-controllability of the linear system (2.5). That is, for any vector $d \in \mathbb{R}^{r}$ there exists a vector $\alpha \in \mathbb{R}^{n}$ and a function $v(\cdot) \in \mathrm{C}_{\mathrm{p}}\left([a, b], \mathbb{R}^{m}\right)$ with $N(t) v(t)=0$ for all $t \in[a, b]$ such that the solution $\eta(\cdot)$ of the initial value problem (2.5)(i) with $\eta(a)=\alpha$ 
satisfies $M\left(\eta^{T}(a), \eta^{T}(b)\right)^{T}=d$. Furthermore, by Proposition 4.6 in [17], the $M$-controllability of system (2.5) is equivalent to the condition

$$
\left\{\mathcal{E}^{T}(t) \gamma=0, \quad t \in[a, b], \quad \mathcal{D}^{T} \gamma=0, \quad \gamma \in \mathbb{R}^{r}\right\} \quad \Rightarrow \quad \gamma=0
$$

where the matrices $\mathcal{E}(\cdot) \in \mathrm{C}_{\mathrm{p}}\left([a, b], \mathbb{R}^{r \times(m-k)}\right)$ and $\mathcal{D} \in \mathbb{R}^{r \times n}$ are defined by

$$
\mathcal{E}(t):=M_{b} \Phi(b) \Phi^{-1}(t) \mathcal{B}(t) Y(t), \quad t \in[a, b], \quad \mathcal{D}:=M_{a}+M_{b} \Phi(b),
$$

and $\Phi(\cdot) \in \mathrm{C}_{\mathrm{p}}^{1}\left([a, b], \mathbb{R}^{n \times n}\right)$ is the fundamental matrix of the system $\dot{\Phi}=\mathcal{A}(t) \Phi$ for $t \in[a, b]$ with $\Phi(a)=I$. Note that $\left(\Phi^{T}\right)^{-1}(\cdot)$ is the fundamental matrix of the homogeneous part of the adjoint equation (2.8).

Next we define the second variation of the functional $J$ in problem $(\mathrm{C})$.

Definition 2.7. Assume that (A2) holds at $(x(\cdot), u(\cdot))$ and that problem (C) is normal at the feasible pair $(x(\cdot), u(\cdot))$ satisfying the weak Pontryagin principle. Let $p(\cdot), \lambda(\cdot)$, and $\lambda_{0}=1$ be the corresponding multipliers from Definition 2.2. The second variation $J^{\prime \prime}$ of $J$ is the quadratic functional defined to be

$$
J^{\prime \prime}(\eta, v):=\frac{1}{2}\left(\begin{array}{l}
\eta(a) \\
\eta(b)
\end{array}\right)^{T} \Gamma\left(\begin{array}{l}
\eta(a) \\
\eta(b)
\end{array}\right)+\frac{1}{2} \int_{a}^{b}\left(\begin{array}{l}
\eta(t) \\
v(t)
\end{array}\right)^{T} \nabla_{(x, u)}^{2} H(t)\left(\begin{array}{l}
\eta(t) \\
v(t)
\end{array}\right) \mathrm{d} t,
$$

where $(\eta(\cdot), v(\cdot))$ is admissible for problem $(\mathrm{C})$, that is, the pair $(\eta(\cdot), v(\cdot)) \in \mathrm{C}_{\mathrm{p}}^{1}\left([a, b], \mathbb{R}^{n}\right) \times \mathrm{C}_{\mathrm{p}}\left([a, b], \mathbb{R}^{m}\right)$ satisfies condition (2.5). Here, the matrix $\Gamma \in \mathbb{R}^{2 n \times 2 n}$ is defined by

$$
\Gamma:=\nabla^{2} K(x(a), x(b))+\gamma^{T} \nabla^{2} \varphi(x(a), x(b))
$$

and

$$
\nabla_{(x, u)}^{2} H(t)=\left(\begin{array}{cc}
P(t) & Q(t) \\
Q^{T}(t) & R(t)
\end{array}\right):=\left(\begin{array}{cc}
H_{x x}(t) & H_{x u}(t) \\
H_{u x}(t) & H_{u u}(t)
\end{array}\right)
$$

where in (2.17) the second order partial derivatives of the Hamiltonian $H$ are evaluated at the argument $(t, x(t), u(t), p(t), \lambda(t), 1)$. Note that the functions $P(\cdot), Q(\cdot)$, and $R(\cdot)$ satisfy $P(\cdot) \in \mathrm{C}_{\mathrm{p}}\left([a, b], \mathbb{R}^{n \times n}\right), Q(\cdot) \in$ $\mathrm{C}_{\mathrm{p}}\left([a, b], \mathbb{R}^{n \times m}\right)$, and $R(\cdot) \in \mathrm{C}_{\mathrm{p}}\left([a, b], \mathbb{R}^{m \times m}\right)$.

We say that the functional $J^{\prime \prime}$ is nonnegative if $J^{\prime \prime}(\eta, v) \geq 0$ for all admissible pairs $(\eta(\cdot), v(\cdot))$ on $[a, b]$. The functional $J^{\prime \prime}$ is coercive if there exists $\alpha>0$ such that

$$
J^{\prime \prime}(\eta, v) \geq \frac{\alpha}{2}\left\{|\eta(a)|^{2}+|\eta(b)|^{2}+\int_{a}^{b}\left\{|\eta(t)|^{2}+|v(t)|^{2}\right\} \mathrm{d} t\right\}
$$

for all pairs $(\eta(\cdot), v(\cdot))$, which are admissible for problem $(\mathrm{C})$.

Remark 2.8. Since the space of continuous functions on $[a, b]$ is dense in $L^{2}[a, b]$, the nonnegativity (resp., coercivity) of $J^{\prime \prime}(\eta, v)$ over all $(\eta(\cdot), v(\cdot)) \in \mathrm{C}_{\mathrm{p}}^{1}\left([a, b], \mathbb{R}^{n}\right) \times \mathrm{C}_{\mathrm{p}}\left([a, b], \mathbb{R}^{m}\right)$ that are admissible for (C) is actually equivalent to the nonnegativity (resp., coercivity) of $J^{\prime \prime}(\eta, v)$ over all $(\eta(\cdot), v(\cdot)) \in W^{1,2}\left([a, b], \mathbb{R}^{n}\right) \times L^{2}\left([a, b], \mathbb{R}^{m}\right)$ satisfying (2.5) for almost all $t \in[a, b]$.

The Legendre-Clebsch condition for problem (C) is defined as

$$
Y^{T}(t) R(t) Y(t) \geq 0, \quad t \in[a, b]
$$


and the strengthened Legendre-Clebsch condition for problem (C) is

$$
Y^{T}(t) R(t) Y(t)>0, \quad t \in[a, b],
$$

where we recall that $Y(\cdot) \in \mathrm{C}_{\mathrm{p}}\left([a, b], \mathbb{R}^{m \times(m-k)}\right)$ is the matrix function for which the columns of $Y(t)$ form an orthonormal basis for the kernel of $N(t)$ on $[a, b]$. In other words, these conditions state that the matrix $R(t)$ defined in (2.17) is positive semidefinite, respectively, positive definite on the kernel of $N(t)$ for all $t \in[a, b]$.

Remark 2.9. If the second variation $J^{\prime \prime}$ is nonnegative, then the Legendre-Clebsch condition (2.19) holds. If, however, the second variation $J^{\prime \prime}$ is coercive, then the strengthened Legendre-Clebsch condition (2.20) is satisfied. This follows from Theorems 4.1.1 and 4.1.2 of [18] after replacing in $J^{\prime \prime}(\eta, v)$ and in the admissible equation the control function $v(\cdot)$ by the formula $v(t)=Y(t) w(t)$ for all $t \in[a, b]$, where $w(\cdot) \in \mathrm{C}_{\mathrm{p}}\left([a, b], \mathbb{R}^{m-k}\right)$ is the new unconstrained control. The resulting coefficients in (2.17) and (2.5) are then

$$
\left(\begin{array}{cc}
P(t) & Q(t) Y(t) \\
Y^{T}(t) Q^{T}(t) & Y^{T}(t) R(t) Y(t)
\end{array}\right) \quad \text { and } \quad(\mathcal{A}(t), \mathcal{B}(t) Y(t)) .
$$

With problem (C) and a normal feasible pair $(x(\cdot), u(\cdot))$ we associate the Jacobi system (or the linear Hamiltonian system)

$$
\dot{\eta}=A(t) \eta+B(t) \xi, \quad \dot{\xi}=C(t) \eta-A^{T}(t) \xi, \quad t \in[a, b]
$$

where $\eta(\cdot), \xi(\cdot) \in \mathrm{C}_{\mathrm{p}}^{1}\left([a, b], \mathbb{R}^{n}\right)$ and the coefficients $A(\cdot), B(\cdot), C(\cdot) \in \mathrm{C}_{\mathrm{p}}\left([a, b], \mathbb{R}^{n \times n}\right)$ are given on the interval $[a, b]$ by the formulas

$$
\left.\begin{array}{l}
A(t):=\mathcal{A}(t)-\mathcal{B}(t) Y(t)\left[Y^{T}(t) R(t) Y(t)\right]^{-1} Y^{T}(t) Q^{T}(t), \\
B(t):=\mathcal{B}(t) Y(t)\left[Y^{T}(t) R(t) Y(t)\right]^{-1} Y^{T}(t) \mathcal{B}^{T}(t), \\
C(t):=P(t)-Q(t) Y(t)\left[Y^{T}(t) R(t) Y(t)\right]^{-1} Y^{T}(t) Q^{T}(t)
\end{array}\right\}
$$

with $\mathcal{A}(t), \mathcal{B}(t), Y(t), P(t), Q(t), R(t)$ defined in (2.6) and (2.17). In the above formulas we assume that the matrix $Y^{T}(t) R(t) Y(t)$ is invertible on $[a, b]$. This property is a consequence of the strengthened Legendre condition (2.20). Note that the adjoint variable $\xi(t)$ is taken to be the negative of the corresponding adjoint variable obtained from the weak Pontryagin principle, when it is applied to the second variation $\mathcal{J}^{\prime \prime}(\eta, v)$.

Remark 2.10. Straightforward calculations show that the only solution $p(\cdot)$ of the system

$$
-p^{\prime}=\mathcal{A}^{T}(t) p, \quad Y^{T}(t) \mathcal{B}^{T}(t) p=0, \quad t \in[a, b], \quad\left(\begin{array}{c}
-p(a) \\
p(b)
\end{array}\right)=M^{T} \gamma
$$

for some $\gamma \in \mathbb{R}^{r}$ is the trivial solution $p(t) \equiv 0$ on $[a, b]$ if and only if the only solution $\xi(\cdot)$ of

$$
\xi^{\prime}=-A^{T}(t) \xi, \quad B(t) \xi=0, \quad t \in[a, b], \quad\left(\begin{array}{c}
\xi(a) \\
-\xi(b)
\end{array}\right)=M^{T} \omega
$$

for some $\omega \in \mathbb{R}^{r}$ is the trivial solution $\xi(t) \equiv 0$ on $[a, b]$. The matrices $A(t)$ and $B(t)$ appearing in $(2.24)$ are defined by (2.22). This means that, under the assumption that the matrix $Y^{T}(t) R(t) Y(t)$ is invertible on $[a, b]$, the normality of problem $(\mathrm{C})$ is equivalent to the normality of the corresponding accessory problem (i.e., minimizing the second variation $\left.\mathcal{J}^{\prime \prime}(\eta, v)\right)$. 


\section{TRANSFORMATION TO SEPARATED STATE ENDPOINTS}

Following (1.1) we denote the midpoint of the interval $[a, b]$ by $c$. Together with problem $(\mathrm{C})$ we consider the augmented nonlinear optimal control problem over the interval $[c, b]$ with separated endpoints

$$
\operatorname{minimize} \quad \tilde{J}(\tilde{x}, \tilde{u}):=\tilde{K}_{c}(\tilde{x}(c))+\tilde{K}_{b}(\tilde{x}(b))+\int_{c}^{b} \tilde{L}(t, \tilde{x}(t), \tilde{u}(t)) \mathrm{d} t
$$

subject to $\tilde{x}(\cdot) \in \mathrm{C}_{\mathrm{p}}^{1}\left([c, b], \mathbb{R}^{2 n}\right)$ and $\tilde{u}(\cdot) \in \mathrm{C}_{\mathrm{p}}\left([c, b], \mathbb{R}^{2 m}\right)$ such that

$$
\begin{gathered}
\dot{\tilde{x}}(t)=\tilde{f}(t, \tilde{x}(t), \tilde{u}(t)), \quad t \in[c, b], \\
\tilde{\psi}(t, \tilde{u}(t))=0, \quad t \in[c, b], \\
\tilde{\varphi}_{c}(\tilde{x}(c))=0, \quad \tilde{\varphi}_{b}(\tilde{x}(b))=0 .
\end{gathered}
$$

Given the data $(L, f, \psi, K, \varphi)$ of problem $(\mathrm{C})$ we define the corresponding data of problem $(\tilde{\mathrm{C}})$ by the functions

$$
\begin{array}{lll}
\tilde{L}:[c, b] \times \mathbb{R}^{2 n} \times \mathbb{R}^{2 m} \rightarrow \mathbb{R}, & \tilde{K}_{c}: \mathbb{R}^{2 n} \rightarrow \mathbb{R}, \quad \tilde{K}_{b}: \mathbb{R}^{2 n} \rightarrow \mathbb{R}, \\
\tilde{f}:[c, b] \times \mathbb{R}^{2 n} \times \mathbb{R}^{2 m} \rightarrow \mathbb{R}^{2 n}, & \tilde{\varphi}_{c}: \mathbb{R}^{2 n} \rightarrow \mathbb{R}^{n}, & \tilde{\varphi}_{b}: \mathbb{R}^{2 n} \rightarrow \mathbb{R}^{r}, \\
\tilde{\psi}:[c, b] \times \mathbb{R}^{2 m} \rightarrow \mathbb{R}^{2 k}, &
\end{array}
$$

where

$$
\left.\begin{array}{c}
\tilde{L}(t, \tilde{x}, \tilde{u}):=L\left(a+b-t, \tilde{x}_{1},-\tilde{u}_{1}\right)+L\left(t, \tilde{x}_{2}, \tilde{u}_{2}\right), \\
\tilde{f}(t, \tilde{x}, \tilde{u}):=\left(\begin{array}{c}
-f\left(a+b-t, \tilde{x}_{1},-\tilde{u}_{1}\right) \\
f\left(t, \tilde{x}_{2}, \tilde{u}_{2}\right)
\end{array}\right), \quad \tilde{\psi}(t, \tilde{u}):=\left(\begin{array}{c}
\psi\left(a+b-t,-\tilde{u}_{1}\right) \\
\psi\left(t, \tilde{u}_{2}\right)
\end{array}\right), \\
\tilde{K}_{c}(\tilde{x}):=0 \quad \tilde{K}_{b}(\tilde{x}):=K\left(\tilde{x}_{1}, \tilde{x}_{2}\right), \quad \tilde{\varphi}_{c}(\tilde{x}):=\tilde{x}_{1}-\tilde{x}_{2}, \quad \tilde{\varphi}_{b}(\tilde{x}):=\varphi\left(\tilde{x}_{1}, \tilde{x}_{2}\right) .
\end{array}\right\}
$$

We split the augmented variables $\tilde{x}, \tilde{u}, \tilde{p}, \tilde{\lambda}, \tilde{\gamma}$, and the augmented functions $\tilde{f}(\cdot, \cdot, \cdot)$ and $\tilde{\psi}(\cdot, \cdot)$ into two entries, whose dimensions are compatible with problem (C), namely

$$
\left.\begin{array}{r}
\tilde{x}=\left(\begin{array}{c}
\tilde{x}_{1} \\
\tilde{x}_{2}
\end{array}\right), \quad \tilde{u}=\left(\begin{array}{c}
\tilde{u}_{1} \\
\tilde{u}_{2}
\end{array}\right), \quad \tilde{p}=\left(\begin{array}{c}
\tilde{p}_{1} \\
\tilde{p}_{2}
\end{array}\right), \quad \tilde{\lambda}=\left(\begin{array}{c}
\tilde{\lambda}_{1} \\
\tilde{\lambda}_{2}
\end{array}\right), \quad \tilde{\gamma}=\left(\begin{array}{c}
\tilde{\gamma}_{1} \\
\tilde{\gamma}_{2}
\end{array}\right), \\
\tilde{f}(t, \tilde{x}, \tilde{u})=\left(\begin{array}{c}
\tilde{f}_{1}(t, \tilde{x}, \tilde{u}) \\
\tilde{f}_{2}(t, \tilde{x}, \tilde{u})
\end{array}\right), \quad \tilde{\psi}(t, \tilde{u})=\left(\begin{array}{c}
\tilde{\psi}_{1}(t, \tilde{u}) \\
\tilde{\psi}_{2}(t, \tilde{u})
\end{array}\right) .
\end{array}\right\}
$$

And for an easy comparison with the original problem (C) we also define the corresponding functions $\tilde{K}$ : $\mathbb{R}^{2 n \times 2 n} \rightarrow \mathbb{R}$ and $\tilde{\varphi}: \mathbb{R}^{2 n \times 2 n} \rightarrow \mathbb{R}^{n+r}$ by

$$
\tilde{K}(\tilde{y}, \tilde{x}):=\tilde{K}_{c}(\tilde{y})+\tilde{K}_{b}(\tilde{x}), \quad \tilde{\varphi}(\tilde{y}, \tilde{x}):=\left(\begin{array}{c}
\tilde{\varphi}_{c}(\tilde{y}) \\
\tilde{\varphi}_{b}(\tilde{x})
\end{array}\right) .
$$

Notice that the ranges of $\tilde{x}(\cdot)$ and $\tilde{u}(\cdot)$ and of the functions $\tilde{f}(\cdot, \cdot, \cdot)$ and $\tilde{\psi}(\cdot, \cdot)$ in problem $(\tilde{\mathrm{C}})$ have double dimensions of the corresponding data for $(\mathrm{C})$. Furthermore, the time interval here is $[c, b]$ compared with the interval $[a, b]$ in problem $(\mathrm{C})$. The definitions of a feasible pair $(\tilde{x}(\cdot), \tilde{u}(\cdot))$ and of a (strict) strong or weak local minimum for problem $(\tilde{\mathrm{C}})$ are analogous to those for problem $(\mathrm{C})$ with the distinction that we now use the norm $\|\tilde{z}(\cdot)\|_{\mathrm{C}_{\mathrm{p}}[c, b]}$. 
When considering for a feasible pair $(\tilde{x}(\cdot), \tilde{u}(\cdot))$ of problem $(\tilde{\mathrm{C}})$ the notions of strong and weak Pontryagin principles stated in Definitions 2.1 and 2.2 , we obtain the augmented multipliers $\tilde{p}(\cdot) \in \mathrm{C}_{\mathrm{p}}^{1}\left([c, b], \mathbb{R}^{2 n}\right), \tilde{\lambda}(\cdot) \in$ $\mathrm{C}_{\mathrm{p}}\left([c, b], \mathbb{R}^{2 k}\right)$, and $\tilde{\gamma} \in \mathbb{R}^{n+r}$. The Hamiltonian

$$
\tilde{H}:[c, b] \times \mathbb{R}^{2 n} \times \mathbb{R}^{2 m} \times \mathbb{R}^{2 n} \times \mathbb{R}^{2 k} \times \mathbb{R} \rightarrow \mathbb{R}
$$

corresponding to problem $(\tilde{\mathrm{C}})$ is defined by

$$
\begin{aligned}
\tilde{H}\left(t, \tilde{x}, \tilde{u}, \tilde{p}, \tilde{\lambda}, \tilde{\lambda}_{0}\right) & :=\tilde{p}^{T} \tilde{f}(t, \tilde{x}, \tilde{u})+\tilde{\lambda}_{0} \tilde{L}(t, \tilde{x}, \tilde{u})+\tilde{\lambda}^{T} \tilde{\psi}(t, \tilde{u}) \\
& \stackrel{(3.4)}{=} H\left(a+b-t, \tilde{x}_{1},-\tilde{u}_{1},-\tilde{p}_{1}, \tilde{\lambda}_{1}, \tilde{\lambda}_{0}\right)+H\left(t, \tilde{x}_{2}, \tilde{u}_{2}, \tilde{p}_{2}, \tilde{\lambda}_{2}, \tilde{\lambda}_{0}\right),
\end{aligned}
$$

where we used the standard partitions from (3.5). The gradients of the functions in (3.4) are

$$
\begin{aligned}
\tilde{f}_{\tilde{x}}(t, \tilde{x}, \tilde{u}) & =\left(\begin{array}{cc}
-f_{x}\left(a+b-t, \tilde{x}_{1},-\tilde{u}_{1}\right) & 0 \\
0 & f_{x}\left(t, \tilde{x}_{2}, \tilde{u}_{2}\right)
\end{array}\right), \\
\tilde{f}_{\tilde{u}}(t, \tilde{x}, \tilde{u}) & =\left(\begin{array}{cc}
f_{u}\left(a+b-t, \tilde{x}_{1},-\tilde{u}_{1}\right) & 0 \\
0 & f_{u}\left(t, \tilde{x}_{2}, \tilde{u}_{2}\right)
\end{array}\right) \\
\tilde{\psi}_{\tilde{u}}(t, \tilde{u}) & =\left(\begin{array}{cc}
-\psi_{u}\left(a+b-t,-\tilde{u}_{1}\right) & 0 \\
0 & \psi_{u}\left(t, \tilde{u}_{2}\right)
\end{array}\right) \\
\tilde{L}_{\tilde{x}}(t, \tilde{x}, \tilde{u}) & =\left(\begin{array}{cc}
L_{x}\left(a+b-t, \tilde{x}_{1},-\tilde{u}_{1}\right) & L_{x}\left(t, \tilde{x}_{2}, \tilde{u}_{2}\right)
\end{array}\right), \\
\tilde{L}_{\tilde{u}}(t, \tilde{x}, \tilde{u}) & =\left(\begin{array}{ll}
-L_{u}\left(a+b-t, \tilde{x}_{1},-\tilde{u}_{1}\right) & L_{u}\left(t, \tilde{x}_{2}, \tilde{u}_{2}\right)
\end{array}\right) \\
\nabla \tilde{\varphi}_{c}(\tilde{x}) & =(I,-I), \quad \nabla \tilde{\varphi}_{b}(\tilde{x})=\nabla \varphi\left(\tilde{x}_{1}, \tilde{x}_{2}\right) \\
\nabla \tilde{K}_{c}(\tilde{x}) & =0, \quad \nabla \tilde{K}_{b}(\tilde{x})=\nabla K\left(\tilde{x}_{1}, \tilde{x}_{2}\right) .
\end{aligned}
$$

Given the data and a feasible pair $(x(\cdot), u(\cdot))$ for problem $(\mathrm{C})$, we define in accordance with notation $(3.5)$ for $t \in[c, b]$ the augmented pair $(\tilde{x}(\cdot), \tilde{u}(\cdot))$ by

$$
\tilde{x}(t):=\left(\begin{array}{c}
x(a+b-t) \\
x(t)
\end{array}\right), \quad \tilde{u}(t):=\left(\begin{array}{c}
-u(a+b-t) \\
u(t)
\end{array}\right), \quad t \in[c, b] .
$$

Conversely, given the data of problem $(\tilde{\mathrm{C}})$ in $(3.4)$ and a feasible pair $(\tilde{x}(\cdot), \tilde{u}(\cdot))$ for $(\tilde{\mathrm{C}})$ we define for $t \in[a, b]$ the functions

$$
x(t):=\left\{\begin{array}{ll}
\tilde{x}_{1}(a+b-t), & t \in[a, c], \\
\tilde{x}_{2}(t), & t \in[c, b],
\end{array} \quad u(t):= \begin{cases}-\tilde{u}_{1}(a+b-t), & t \in[a, c], \\
\tilde{u}_{2}(t), & t \in[c, b] .\end{cases}\right.
$$

In the next two results we make precise the relationship regarding the feasibility and the local (strong or weak) optimality between the pairs $(x(\cdot), u(\cdot))$ and $(\tilde{x}(\cdot), \tilde{u}(\cdot))$ in their respective problems $(\mathrm{C})$ and $(\tilde{\mathrm{C}})$. Here the data of $(\tilde{\mathrm{C}})$ is obtained via those of $(\mathrm{C})$ through equations (3.4) and (3.6).

Proposition 3.1 (Feasibility). If a pair $(x(\cdot), u(\cdot))$ is feasible for problem $(\mathrm{C})$, then the pair $(\tilde{x}(\cdot), \tilde{u}(\cdot))$ defined by (3.10) is feasible for problem $(\tilde{\mathrm{C}})$ with the objective value $\tilde{J}(\tilde{x}, \tilde{u})=J(x, u)$. Conversely, if a pair $(\tilde{x}(\cdot), \tilde{u}(\cdot))$ is feasible for problem $(\tilde{\mathrm{C}})$, then the pair $(x(\cdot), u(\cdot))$ defined by $(3.11)$ is feasible for problem $(\mathrm{C})$ with the objective value $J(x, u)=\tilde{J}(\tilde{x}, \tilde{u})$. 
Proof. Let $(x(\cdot), u(\cdot))$ be a feasible pair for problem (C) and define $(\tilde{x}(\cdot), \tilde{u}(\cdot))$ by $(3.10)$. Then we obviously have $\tilde{x}(\cdot) \in \mathrm{C}_{\mathrm{p}}^{1}\left([c, b], \mathbb{R}^{2 n}\right)$ and $\tilde{u}(\cdot) \in \mathrm{C}_{\mathrm{p}}\left([c, b], \mathbb{R}^{2 m}\right)$. Moreover,

$$
\begin{gathered}
\dot{\tilde{x}}(t) \stackrel{(3.10)}{=}\left(\begin{array}{c}
-\dot{x}(a+b-t) \\
\dot{x}(t)
\end{array}\right) \stackrel{(2.1)}{=}\left(\begin{array}{c}
-f(a+b-t, x(a+b-t), u(a+b-t)) \\
f(t, x(t), u(t))
\end{array}\right) \\
\stackrel{(3.10)}{=}\left(\begin{array}{c}
-f\left(a+b-t, \tilde{x}_{1}(t),-\tilde{u}_{1}(t)\right) \\
f\left(t, \tilde{x}_{2}(t), \tilde{u}_{2}(t)\right)
\end{array}\right) \stackrel{(3.4)}{=} \tilde{f}(t, \tilde{x}(t), \tilde{u}(t)), \\
\tilde{\psi}(t, \tilde{u}(t)) \stackrel{(3.4)}{=}\left(\begin{array}{c}
\psi\left(a+b-t,-\tilde{u}_{1}(t)\right) \\
\psi\left(t, \tilde{u}_{2}(t)\right)
\end{array}\right) \stackrel{(3.10)}{=}\left(\begin{array}{c}
\psi(a+b-t, u(a+b-t)) \\
\psi(t, u(t))
\end{array}\right) \stackrel{(2.2)}{=} 0,
\end{gathered}
$$

hold for every $t \in[c, b]$, and

$$
\begin{aligned}
& \tilde{\varphi}_{c}(\tilde{x}(c)) \stackrel{(3.4)}{=} \tilde{x}_{1}(c)-\tilde{x}_{2}(c) \stackrel{(3.10)}{=} x(c)-x(c)=0, \\
& \tilde{\varphi}_{b}(\tilde{x}(b)) \stackrel{(3.4)}{=} \varphi\left(\tilde{x}_{1}(b), \tilde{x}_{2}(b)\right) \stackrel{(3.10)}{=} \varphi(x(a), x(b)) \stackrel{(2.3)}{=} 0 .
\end{aligned}
$$

Therefore, the pair $(\tilde{x}(\cdot), \tilde{u}(\cdot))$ satisfies constraints $(3.1)-(3.3)$ and hence it is feasible for problem $(\tilde{\mathrm{C}})$. In addition,

$$
\begin{aligned}
\tilde{J}(\tilde{x}, \tilde{u}) \stackrel{(3.4)}{=} & K\left(\tilde{x}_{1}(b), \tilde{x}_{2}(b)\right)+\int_{c}^{b}\left\{L\left(a+b-t, \tilde{x}_{1}(t),-\tilde{u}_{1}(t)\right)+L\left(t, \tilde{x}_{2}(t), \tilde{u}_{2}(t)\right)\right\} \mathrm{d} t \\
\stackrel{(3.10)}{=} & K(x(a), x(b))+\int_{c}^{b} L(a+b-t, x(a+b-t), u(a+b-t)) \mathrm{d} t \\
& \quad+\int_{c}^{b} L(t, x(t), u(t)) \mathrm{d} t \\
= & K(x(a), x(b))+\int_{c}^{a} L(s, x(s), u(s))(-\mathrm{d} s)+\int_{c}^{b} L(t, x(t), u(t)) \mathrm{d} t \\
= & K(x(a), x(b))+\int_{a}^{b} L(t, x(t), u(t)) \mathrm{d} t=J(x, u),
\end{aligned}
$$

where we substituted $s:=a+b-t$ in the first integral of the second equation.

Conversely, assume that $(\tilde{x}(\cdot), \tilde{u}(\cdot))$ is a feasible pair for problem $(\tilde{\mathrm{C}})$ and define $(x(\cdot), u(\cdot))$ by $(3.11)$. Then we have $u(\cdot) \in \mathrm{C}_{\mathrm{p}}\left([a, b], \mathbb{R}^{m}\right)$. Moreover, the first equality in (3.3) and the second last equality in (3.4) imply that $\tilde{x}_{1}(c)=\tilde{x}_{2}(c)$, so that $x(\cdot) \in \mathrm{C}_{\mathrm{p}}^{1}\left([a, b], \mathbb{R}^{n}\right)$. For $t \in[a, c]$ we calculate

$$
\begin{gathered}
\dot{x}(t) \stackrel{(3.11)}{=}-\dot{\tilde{x}}_{1}(a+b-t) \stackrel{(3.1)}{=}-\tilde{f}_{1}(a+b-t, \tilde{x}(a+b-t), \tilde{u}(a+b-t)) \\
\stackrel{(3.4)}{=} f\left(t, \tilde{x}_{1}(a+b-t),-\tilde{u}_{1}(a+b-t)\right) \stackrel{(3.11)}{=} f(t, x(t), u(t)),
\end{gathered}
$$

while for $t \in[c, b]$ we calculate

$$
\dot{x}(t) \stackrel{(3.11)}{=} \dot{\tilde{x}}_{2}(t) \stackrel{(3.1)}{=} \tilde{f}_{2}(t, \tilde{x}(t), \tilde{u}(t)) \stackrel{(3.4)}{=} f\left(t, \tilde{x}_{2}(t), \tilde{u}_{2}(t)\right) \stackrel{(3.11)}{=} f(t, x(t), u(t)) .
$$

Therefore, constraint (2.1) is satisfied. Similarly, for $t \in[a, c]$ we have

$$
\psi(t, u(t)) \stackrel{(3.11)}{=} \psi\left(t,-\tilde{u}_{1}(a+b-t)\right) \stackrel{(3.4)}{=} \tilde{\psi}_{1}(a+b-t, \tilde{u}(a+b-t)) \stackrel{(3.2)}{=} 0,
$$


while for $t \in[c, b]$ we have

$$
\psi(t, u(t)) \stackrel{(3.11)}{=} \psi\left(t, \tilde{u}_{2}(t)\right) \stackrel{(3.4)}{=} \tilde{\psi}_{2}(t, \tilde{u}(t)) \stackrel{(3.2)}{=} 0 .
$$

This shows that constraint (2.2) holds. The validity of constraint (2.3) is verified by

$$
\varphi(x(a), x(b)) \stackrel{(3.11)}{=} \varphi\left(\tilde{x}_{1}(b), \tilde{x}_{2}(b)\right) \stackrel{(3.4)}{=} \tilde{\varphi}_{b}(\tilde{x}(b)) \stackrel{(3.3)}{=} 0 .
$$

Thus, we conclude that the pair $(x(\cdot), u(\cdot))$ is feasible for problem $(\mathrm{C})$. Finally, we have

$$
\begin{aligned}
J(x, u)= & K(x(a), x(b))+\int_{a}^{c} L(t, x(t), u(t)) \mathrm{d} t+\int_{c}^{b} L(t, x(t), u(t)) \mathrm{d} t \\
\stackrel{(3.11)}{=} K\left(\tilde{x}_{1}(b), \tilde{x}_{2}(b)\right)+\int_{a}^{c} L\left(t, \tilde{x}_{1}(a+b-t),-\tilde{u}_{1}(a+b-t)\right) \mathrm{d} t & \\
& \quad+\int_{c}^{b} L\left(t, \tilde{x}_{2}(t), \tilde{u}_{2}(t)\right) \mathrm{d} t \\
= & K\left(\tilde{x}_{1}(b), \tilde{x}_{2}(b)\right)+\int_{c}^{b}\left\{L\left(a+b-s, \tilde{x}_{1}(s),-\tilde{u}_{1}(s)\right)+L\left(s, \tilde{x}_{2}(s), \tilde{u}_{2}(s)\right)\right\} \mathrm{d} s \\
\stackrel{(3.4)}{=} & \tilde{K}_{c}(\tilde{x}(c))+\tilde{K}_{b}(\tilde{x}(b))+\int_{c}^{b} \tilde{L}(s, \tilde{x}(s), \tilde{u}(s)) \mathrm{d} s=\tilde{J}(\tilde{x}, \tilde{u}),
\end{aligned}
$$

where we substituted $s:=a+b-t$ in the first integral of the second equation.

Corollary 3.2 (Strong and weak local optimality). If $(x(\cdot), u(\cdot))$ is a (strict) strong, respectively, (strict) weak local minimum for problem $(\mathrm{C})$, then $(\tilde{x}(\cdot), \tilde{u}(\cdot))$ defined by $(3.10)$ is a (strict) strong, respectively, (strict) weak local minimum for problem $(\tilde{\mathrm{C}})$. Conversely, if $(\tilde{x}(\cdot), \tilde{u}(\cdot))$ is a (strict) strong, respectively, (strict) weak local minimum for problem $(\tilde{\mathrm{C}})$, then $(x(\cdot), u(\cdot))$ defined by (3.11) is a (strict) strong, respectively, (strict) weak local minimum for problem $(\mathrm{C})$.

Proof. The result follows from Proposition 3.1, since the values of $J(x, u)$ and $\tilde{J}(\tilde{x}, \tilde{u})$ are the same under the definitions in (3.10) and (3.11).

Next we analyze the correspondence of the strong Pontryagin principle or the weak Pontryagin principle between the problems $(\mathrm{C})$ and $(\tilde{\mathrm{C}})$. Assume that a pair $(x(\cdot), u(\cdot))$ is feasible for problem $(\mathrm{C})$ and satisfies the strong, respectively, the weak Pontryagin principle for problem (C) with multipliers $\lambda_{0} \geq 0, \gamma \in \mathbb{R}^{r}, p(\cdot) \in$ $\mathrm{C}_{\mathrm{p}}^{1}\left([a, b], \mathbb{R}^{n}\right)$, and $\lambda(\cdot) \in \mathrm{C}_{\mathrm{p}}\left([a, b], \mathbb{R}^{k}\right)$ satisfying conditions (2.7)-(2.10) of Definition 2.1, respectively, conditions (2.7)-(2.9) and (2.11) of Definition 2.2. To these multipliers we associate the "augmented" multipliers defined by

$$
\tilde{\lambda}_{0}:=\lambda_{0}, \quad \tilde{\gamma}:=\left(\begin{array}{c}
p(c) \\
\gamma
\end{array}\right), \quad \tilde{p}(t):=\left(\begin{array}{c}
-p(a+b-t) \\
p(t)
\end{array}\right), \quad \tilde{\lambda}(t):=\left(\begin{array}{c}
\lambda(a+b-t) \\
\lambda(t)
\end{array}\right)
$$

Conversely, according to Definition 2.1, a feasible pair $(\tilde{x}(\cdot), \tilde{u}(\cdot))$ for problem $(\tilde{\mathrm{C}})$ satisfies the strong Pontryagin principle for problem $(\tilde{\mathrm{C}})$ if there exist a constant $\tilde{\lambda}_{0} \geq 0, \tilde{\gamma} \in \mathbb{R}^{n+r}$, and $\tilde{p}(\cdot) \in \mathrm{C}_{\mathrm{p}}^{1}\left([c, b], \mathbb{R}^{2 n}\right)$ satisfying the nondegeneracy condition

$$
\tilde{\lambda}_{0}+\|\tilde{p}(\cdot)\|_{\mathrm{C}_{\mathrm{p}}[c, b]} \neq 0
$$


the adjoint equation

$$
-\dot{\tilde{p}}(t)=\tilde{\mathcal{A}}^{T}(t) \tilde{p}(t)+\tilde{\lambda}_{0} \tilde{L}_{\tilde{x}}^{T}(t, \tilde{x}(t), \tilde{u}(t)), \quad t \in[c, b],
$$

the transversality condition

$$
\left(\begin{array}{c}
-\tilde{p}(c) \\
\tilde{p}(b)
\end{array}\right)=\tilde{\lambda}_{0} \nabla \tilde{K}^{T}(\tilde{x}(c), \tilde{x}(b))+\tilde{M}^{T} \tilde{\gamma}
$$

and the minimality condition

$$
\min _{\tilde{u} \in \mathbb{R}^{2 m}, \tilde{\psi}(t, \tilde{u})=0} \tilde{H}\left(t, \tilde{x}(t), \tilde{u}, \tilde{p}(t), 0, \tilde{\lambda}_{0}\right) \quad \text { is attained at } \tilde{u}(t), t \in[c, b] .
$$

On the other hand, corresponding to Definition 2.2 , a feasible pair $(\tilde{x}(\cdot), \tilde{u}(\cdot))$ is said to satisfy the weak Pontryagin principle for problem ( $\tilde{\mathrm{C}})$ if there exist $\tilde{\lambda}_{0} \geq 0, \tilde{\gamma} \in \mathbb{R}^{n+r}$, and functions $\tilde{p}(\cdot) \in \mathrm{C}_{\mathrm{p}}^{1}\left([c, b], \mathbb{R}^{2 n}\right)$ and $\tilde{\lambda}(\cdot) \in \mathrm{C}_{\mathrm{p}}\left([c, b], \mathbb{R}^{2 k}\right)$ satisfying in addition to (3.13)-(3.15) the stationarity condition

$$
\tilde{\mathcal{B}}^{T}(t) \tilde{p}(t)+\tilde{\lambda}_{0} \tilde{L}_{\tilde{u}}^{T}(t, \tilde{x}(t), \tilde{u}(t))+\tilde{N}^{T}(t) \tilde{\lambda}(t)=0, \quad t \in[c, b]
$$

where according to $(2.6)$ the matrices $\tilde{\mathcal{A}}(t), \tilde{\mathcal{B}}(t), \tilde{N}(t)$ for $t \in[c, b]$ and $\tilde{M}$ are defined by

$$
\left.\begin{array}{rlrl}
\tilde{\mathcal{A}}(t) & :=\tilde{f}_{\tilde{x}}(t, \tilde{x}(t), \tilde{u}(t)), & \tilde{\mathcal{B}}(t) & :=\tilde{f}_{\tilde{u}}(t, \tilde{x}(t), \tilde{u}(t)), \\
\tilde{N}(t) & :=\tilde{\psi}_{\tilde{u}}(t, \tilde{u}(t)), & \tilde{M} & :=\nabla \tilde{\varphi}(\tilde{x}(c), \tilde{x}(b)) .
\end{array}\right\}
$$

Taking into account formulas (3.9) and the definition of the feasible pair $(x(\cdot), u(\cdot))$ for problem $(\mathrm{C})$ corresponding to $(\tilde{x}(\cdot), \tilde{u}(\cdot))$ via $(3.11)$, we obtain the matrices in $(3.18)$ on $[c, b]$ in the form

$$
\begin{aligned}
\tilde{\mathcal{A}}(t)=\left(\begin{array}{cc}
-\mathcal{A}(a+b-t) & 0 \\
0 & \mathcal{A}(t)
\end{array}\right), & \tilde{\mathcal{B}}(t)=\left(\begin{array}{cc}
\mathcal{B}(a+b-t) & 0 \\
0 & \mathcal{B}(t)
\end{array}\right), \\
\tilde{N}(t)=\left(\begin{array}{cc}
-N(a+b-t) & 0 \\
0 & N(t)
\end{array}\right), & \tilde{M}=\left(\begin{array}{ccc}
I & -I & 0 \\
0 & 0 & M
\end{array}\right), \\
\tilde{Y}(t)=\left(\begin{array}{cc}
Y(a+b-t) & 0 \\
0 & Y(t)
\end{array}\right), &
\end{aligned}
$$

where $\tilde{Y}(\cdot) \in \mathrm{C}_{\mathrm{p}}\left([c, b], \mathbb{R}^{2 m \times 2(m-k)}\right)$ satisfies $\tilde{N}(t) \tilde{Y}(t)=0$ and $\tilde{Y}^{T}(t) \tilde{Y}(t)=I_{2(m-k)}$.

To the multipliers $\tilde{\lambda}_{0}, \tilde{\gamma}, \tilde{p}(\cdot)$ satisfying with $(\tilde{x}, \tilde{u})$ equations $(3.13)-(3.16)$, or to the multipliers $\tilde{\lambda}_{0}, \tilde{\gamma}, \tilde{p}(\cdot)$, $\tilde{\lambda}(\cdot)$ satisfying (3.13)-(3.15) and (3.17), we define the corresponding multipliers

$$
\begin{gathered}
\lambda_{0}:=\tilde{\lambda}_{0}, \quad \gamma:=\tilde{\gamma}_{2}, \\
p(t):=\left\{\begin{array}{ll}
-\tilde{p}_{1}(a+b-t), & t \in[a, c], \\
\tilde{p}_{2}(t), & t \in[c, b],
\end{array} \quad \lambda(t):=\left\{\begin{array}{ll}
\tilde{\lambda}_{1}(a+b-t), & t \in[a, c], \\
\tilde{\lambda}_{2}(t), & t \in[c, b],
\end{array}\right\}\right.
\end{gathered}
$$

where we use the standard partitions from (3.5).

Proposition 3.3 (Strong Pontryagin principle). Assume that a feasible pair $(x(\cdot), u(\cdot))$ satisfies the strong Pontryagin principle for problem (C) with multipliers $\lambda_{0}, \gamma$, and $p(\cdot)$. Then the pair $(\tilde{x}(\cdot), \tilde{u}(\cdot))$ defined by 
(3.10) satisfies the strong Pontryagin principle for problem $(\tilde{\mathrm{C}})$ with the multipliers $\tilde{\lambda}_{0}, \tilde{\gamma}, \tilde{p}(\cdot)$ defined in (3.12). Conversely, if a feasible pair $(\tilde{x}(\cdot), \tilde{u}(\cdot))$ satisfies the strong Pontryagin principle for problem $(\tilde{\mathrm{C}})$ with multipliers $\tilde{\lambda}_{0}, \tilde{\gamma}, \tilde{p}(\cdot)$, then the pair $(x(\cdot), u(\cdot))$ defined by (3.11) satisfies the strong Pontryagin principle for problem $(\mathrm{C})$ with the multipliers $\lambda_{0}, \gamma, p(\cdot)$ defined in (3.20).

Proof. Assume that $(2.7)-(2.10)$ hold for a feasible pair $(x(\cdot), u(\cdot))$ with the multipliers $\lambda_{0}, \gamma$, and $p(\cdot)$. Let $(\tilde{x}(\cdot), \tilde{u}(\cdot))$ and $\tilde{\lambda}_{0}, \tilde{\gamma}, \tilde{p}(\cdot)$ be defined by $(3.10)$ and $(3.12)$. Then $(\tilde{x}(\cdot), \tilde{u}(\cdot))$ is feasible for problem $(\tilde{\mathrm{C}})$, by Proposition 3.1, and $\tilde{p}(\cdot) \in \mathrm{C}_{\mathrm{p}}^{1}\left([c, b], \mathbb{R}^{2 n}\right)$. If (3.13) does not hold, then (3.12) yields $\lambda_{0}=0$ and on $[c, b]$ we have $p(a+b-t) \equiv 0$ and $p(t) \equiv 0$. It follows that $\lambda_{0}=0$ and $p(t) \equiv 0$ on $[a, b]$, which contradicts condition (2.7). Therefore, condition (3.13) is satisfied.

Next, for $t \in[c, b]$ and $s:=a+b-t$ we have

$$
\begin{aligned}
&-\dot{\tilde{p}}(t) \stackrel{(3.12)}{=}\left(\begin{array}{c}
-\dot{p}(a+b-t) \\
-\dot{p}(t)
\end{array}\right) \stackrel{(2.8)}{=}\left(\begin{array}{c}
\mathcal{A}^{T}(s) p(s)+\lambda_{0} L_{x}^{T}(s, x(s), u(s)) \\
\mathcal{A}^{T}(t) p(t)+\lambda_{0} L_{x}^{T}(t, x(t), u(t))
\end{array}\right) \\
& \stackrel{(3.10)}{=}\left(\begin{array}{cc}
-\mathcal{A}^{T}(s) & 0 \\
0 & \mathcal{A}^{T}(t)
\end{array}\right)\left(\begin{array}{c}
-p(s) \\
p(t)
\end{array}\right)+\lambda_{0}\left(\begin{array}{c}
L_{x}^{T}\left(s, \tilde{x}_{1}(s),-\tilde{u}_{1}(s)\right) \\
L_{x}^{T}\left(t, \tilde{x}_{2}(t), \tilde{u}_{2}(t)\right)
\end{array}\right) \\
& \stackrel{(3.10),(3.9)}{=} \tilde{\mathcal{A}}^{T}(t) \tilde{p}(t)+\lambda_{0} \tilde{L}_{\tilde{x}}^{T}(t, \tilde{x}(t), \tilde{u}(t)),
\end{aligned}
$$

which shows that condition (3.14) holds. Transversality condition (3.15) is shown to hold by the calculation

$$
\begin{aligned}
\left(\begin{array}{c}
-\tilde{p}(c) \\
\tilde{p}(b)
\end{array}\right) \stackrel{(3.12)}{=}\left(\begin{array}{c}
p(c) \\
-p(c) \\
-p(a) \\
p(b)
\end{array}\right) \stackrel{(2.9)}{=}\left(\begin{array}{c}
p(c) \\
-p(c) \\
\lambda_{0} \nabla K^{T}(x(a), x(b))+M^{T} \gamma
\end{array}\right) \\
\stackrel{(3.9)}{=} \lambda_{0}\left(\begin{array}{cc}
\nabla \tilde{K}_{c}^{T}(\tilde{x}(c)) \\
\nabla \tilde{K}_{b}^{T}(\tilde{x}(b))
\end{array}\right)+\left(\begin{array}{cc}
I & 0 \\
-I & 0 \\
0 & M^{T}
\end{array}\right)\left(\begin{array}{c}
p(c) \\
\gamma
\end{array}\right) \stackrel{(3.6),(3.19)}{=} \tilde{\lambda}_{0} \nabla \tilde{K}^{T}(\tilde{x}(c), \tilde{x}(b))+\tilde{M}^{T} \tilde{\gamma}
\end{aligned}
$$

Now let $t \in[c, b]$ and $\tilde{u} \in \mathbb{R}^{2 m}$ be such that $\tilde{\psi}(t, \tilde{u})=0$. According to the definition of $\tilde{\psi}(\cdot, \cdot)$ in $(3.12)$, this is equivalent to saying that $\psi\left(a+b-t,-\tilde{u}_{1}\right)=0$ and $\psi\left(t, \tilde{u}_{2}\right)=0$. Then, by $(2.10)$ evaluated at $s:=a+b-t$ and at $t$ we have

$$
\begin{aligned}
H\left(s, x(s),-\tilde{u}_{1}, p(s), 0, \lambda_{0}\right) & \geq H\left(s, x(s), u(s), p(s), 0, \lambda_{0}\right) \\
H\left(t, x(t), \tilde{u}_{2}, p(t), 0, \lambda_{0}\right) & \geq H\left(t, x(t), u(t), p(t), 0, \lambda_{0}\right) .
\end{aligned}
$$

Thus, we have

$$
\begin{aligned}
& \tilde{H}\left(t, \tilde{x}(t), \tilde{u}, \tilde{p}(t), 0, \tilde{\lambda}_{0}\right) \stackrel{(3.8),(3.12)}{=} H\left(s, x(s),-\tilde{u}_{1}, p(s), 0, \lambda_{0}\right)+H\left(t, x(t), \tilde{u}_{2}, p(t), 0, \lambda_{0}\right) \\
& \stackrel{(3.21),(3.22)}{\geq} H\left(s, x(s), u(s), p(s), 0, \lambda_{0}\right)+H\left(t, x(t), u(t), p(t), 0, \lambda_{0}\right) \\
& \stackrel{(3.8),(3.12)}{=} \tilde{H}\left(t, \tilde{x}(t), \tilde{u}(t), \tilde{p}(t), 0, \tilde{\lambda}_{0}\right),
\end{aligned}
$$

showing that the minimality condition (3.16) is valid.

Conversely, assume that conditions (3.13)-(3.16) hold for a feasible pair $(\tilde{x}(\cdot), \tilde{u}(\cdot))$ and multipliers $\tilde{\lambda}_{0}, \tilde{\gamma}$, and $\tilde{p}(\cdot)$. Let $(x(\cdot), u(\cdot))$ and $\lambda_{0}, \gamma, p(\cdot)$ be defined by $(3.11)$ and $(3.20)$. Then $(x(\cdot), u(\cdot))$ is feasible for problem $(\mathrm{C})$, 
by Proposition 3.1. From (3.15) we obtain through (3.5), (3.9), (3.11), and (3.19) that

$$
\begin{aligned}
\left(\begin{array}{c}
-\tilde{p}_{1}(c) \\
-\tilde{p}_{2}(c) \\
\tilde{p}(b)
\end{array}\right) & \stackrel{(3.5)}{=}\left(\begin{array}{c}
-\tilde{p}(c) \\
\tilde{p}(b)
\end{array}\right) \stackrel{(3.15),(3.4)}{=} \lambda_{0}\left(\begin{array}{c}
0 \\
\nabla K^{T}\left(\tilde{x}_{1}(b), \tilde{x}_{2}(b)\right)
\end{array}\right)+\left(\begin{array}{cc}
I & 0 \\
-I & 0 \\
0 & M^{T}
\end{array}\right)\left(\begin{array}{c}
\tilde{\gamma}_{1} \\
\tilde{\gamma}_{2}
\end{array}\right) \\
& \stackrel{(3.11)}{=}\left(\begin{array}{c}
\tilde{\gamma}_{1} \\
-\tilde{\gamma}_{1} \\
\lambda_{0} \nabla K^{T}(x(a), x(b))+M^{T} \gamma
\end{array}\right) .
\end{aligned}
$$

Therefore, we have

$$
\tilde{p}_{1}(c)=-\tilde{\gamma}_{1}=-\tilde{p}_{2}(c), \quad\left(\begin{array}{c}
-p(a) \\
p(b)
\end{array}\right) \stackrel{(3.20)}{=}\left(\begin{array}{l}
\tilde{p}_{1}(b) \\
\tilde{p}_{2}(b)
\end{array}\right)=\tilde{p}(b)=\lambda_{0} \nabla K^{T}(x(a), x(b))+M^{T} \gamma
$$

This shows that the function $p(t)$ defined in (3.20) satisfies transversality condition (2.9) and is also continuous at the point $c$, yielding that $p(\cdot) \in \mathrm{C}_{\mathrm{p}}^{1}\left([a, b], \mathbb{R}^{n}\right)$. We now show that adjoint equation (2.8) holds. From adjoint equation (3.14) we get through (3.9), (3.11), (3.19), and (3.20) that for $t \in[c, b]$ and $s:=a+b-t$

$$
\begin{gathered}
\left(\begin{array}{c}
-\dot{p}(s) \\
-\dot{p}(t)
\end{array}\right)=\left(\begin{array}{c}
-\dot{p}(a+b-t) \\
-\dot{p}(t)
\end{array}\right)=\left(\begin{array}{c}
\frac{d}{d t}[p(a+b-t)] \\
-\dot{p}(t)
\end{array}\right) \stackrel{(3.20)}{=}\left(\begin{array}{c}
-\dot{\tilde{p}}_{1}(t) \\
-\dot{\tilde{p}}_{2}(t)
\end{array}\right)=-\dot{\tilde{p}}(t) \\
\stackrel{(3.14)}{=}\left(\begin{array}{c}
-\mathcal{A}^{T}(a+b-t) \tilde{p}_{1}(t) \\
\mathcal{A}^{T}(t) \tilde{p}_{2}(t)
\end{array}\right)+\lambda_{0}\left(\begin{array}{c}
L_{x}^{T}\left(a+b-t, \tilde{x}_{1}(t),-\tilde{u}_{1}(t)\right) \\
L_{x}^{T}\left(t, \tilde{x}_{2}(t), \tilde{u}_{2}(t)\right)
\end{array}\right) \\
\stackrel{(3.20),(3.11)}{=}\left(\begin{array}{c}
\mathcal{A}^{T}(s) p(s) \\
\mathcal{A}^{T}(t) p(t)
\end{array}\right)+\lambda_{0}\left(\begin{array}{c}
L_{x}^{T}(s, x(s), u(s)) \\
L_{x}^{T}(t, x(t), u(t))
\end{array}\right) .
\end{gathered}
$$

Here, the first entry of (3.23) is satisfied for all $s=a+b-t \in[a, c]$, while the second entry of equation (3.23) is satisfied for all $t \in[c, b]$. Therefore, adjoint equation (2.8) holds on $[a, b]$.

If the nondegeneracy condition (2.7) is violated, then $\lambda_{0}=0$ and $p(t) \equiv 0$ on $[a, b]$. From the definition of $\lambda_{0}$ and $p(\cdot)$ in $(3.20)$ it results that $\tilde{\lambda}_{0}=0$, and $\tilde{p}_{1}(t) \equiv 0$ and $\tilde{p}_{2}(t) \equiv 0$ on $[c, b]$, which contradicts (3.13). Hence, (2.7) holds true.

Finally we shall show that the minimality condition (2.10) is satisfied. Using the form the Hamiltonian $\tilde{H}$ in (3.8), the formulas for $\lambda_{0}$ and $p(\cdot)$ in (3.20), and the formulas for $(x(\cdot), u(\cdot))$ in (3.11), it follows that the minimality condition (3.16) is rephrased as follows: for all $t \in[c, b]$, and for $s:=a+b-t$, which is in $[a, c]$, we have

$$
\min _{\substack{\tilde{u}_{1}, \tilde{u}_{2} \in \mathbb{R}^{m} \\ \psi\left(s,-\tilde{u}_{1}\right)=0, \psi\left(t, \tilde{u}_{2}\right)=0}}\left\{H\left(s, \tilde{x}_{1}(s),-\tilde{u}_{1},-\tilde{p}_{1}(s), 0, \tilde{\lambda}_{0}\right)+H\left(t, \tilde{x}_{2}(t), \tilde{u}_{2}, \tilde{p}_{2}(t), 0, \tilde{\lambda}_{0}\right)\right\}
$$

is attained at $\tilde{u}(t)$, where $\tilde{u}(t)=\left(-u^{T}(s), u^{T}(t)\right)^{T}$ according to (3.11). Since in (3.24) the objective function and the constraints are separable in the variables $\tilde{u}_{1}$ and $\tilde{u}_{2}$, it results that the minimization problem in (3.24) is in turn equivalent to two minimization problems, namely, for $t \in[c, b]$ and for $s:=a+b-t$ we have that

$$
\begin{gathered}
\min _{\tilde{u}_{1} \in \mathbb{R}^{m}, \psi\left(s,-\tilde{u}_{1}\right)=0}^{H}\left(s, x(s),-\tilde{u}_{1}, p(s), 0, \lambda_{0}\right) \quad \text { is attained at } u(s) \text { for } s \in[a, c], \\
\min _{\tilde{u}_{2} \in \mathbb{R}^{m}, \psi\left(t, \tilde{u}_{2}\right)=0} H\left(t, x(t), \tilde{u}_{2}, p(t), 0, \lambda_{0}\right) \quad \text { is attained at } u(t) \text { for } t \in[c, b] .
\end{gathered}
$$


Consequently, conditions (3.25) and (3.26) imply that

$$
\min _{u \in \mathbb{R}^{m}, \psi(t, u)=0} H\left(t, x(t), u, p(t), 0, \lambda_{0}\right) \quad \text { is attained at } u(t) \text { for all } t \in[a, b],
$$

which is condition (2.10). This completes the proof of this proposition.

Proposition 3.4 (Weak Pontryagin principle). Assume that a feasible pair $(x(\cdot), u(\cdot))$ satisfies the weak Pontryagin principle for problem $(\mathrm{C})$ with multipliers $\lambda_{0}, \gamma, p(\cdot), \lambda(\cdot)$. Then the pair $(\tilde{x}(\cdot), \tilde{u}(\cdot))$ defined by $(3.10)$ satisfies the weak Pontryagin principle for problem $(\tilde{\mathrm{C}})$ with the multipliers $\tilde{\lambda}_{0}, \tilde{\gamma}, \tilde{p}(\cdot), \tilde{\lambda}(\cdot)$ defined by (3.12). Conversely, if a feasible pair $(\tilde{x}(\cdot) \tilde{u}(\cdot))$ satisfies the weak Pontryagin principle for problem $(\tilde{\mathrm{C}})$ with multipliers $\tilde{\lambda}_{0}, \tilde{\gamma}, \tilde{p}(\cdot), \tilde{\lambda}(\cdot)$, then the pair $(x(\cdot), u(\cdot))$ defined by $(3.11)$ satisfies the weak Pontryagin principle for problem $(\mathrm{C})$ with the multipliers $\lambda_{0}, \gamma, p(\cdot), \lambda(\cdot)$ defined by $(3.20)$.

Proof. Assume that (2.7)-(2.9) and (2.11) hold for a feasible pair $(x(\cdot), u(\cdot))$ with the multipliers $\lambda_{0}, \gamma, p(\cdot)$, and $\lambda(\cdot)$. Let $(\tilde{x}(\cdot), \tilde{u}(\cdot))$ and $\tilde{\lambda}_{0}, \tilde{\gamma}, \tilde{p}(\cdot)$, and $\tilde{\lambda}(\cdot)$ be defined by (3.10) and (3.12). Then, as in the proof of Proposition 3.3, conditions (3.13)-(3.15) are satisfied. It remains to show that also (3.17) is valid. For, let $t \in[c, b]$ and $s:=a+b-t$, then

$$
\begin{aligned}
\tilde{\mathcal{B}}^{T}(t) & \tilde{p}(t)+\tilde{\lambda}_{0} \tilde{L}_{\tilde{u}}^{T}(t, \tilde{x}(t), \tilde{u}(t))+\tilde{N}^{T}(t) \tilde{\lambda}(t) \\
& \stackrel{(3.9)}{=}\left(\begin{array}{cc}
\mathcal{B}^{T}(s) & 0 \\
0 & \mathcal{B}^{T}(t)
\end{array}\right)\left(\begin{array}{c}
-p(s) \\
p(t)
\end{array}\right)+\lambda_{0}\left(\begin{array}{cc}
-L_{u}^{T}(s, x(s), u(s)) \\
L_{u}^{T}(t, x(t), u(t))
\end{array}\right)+\left(\begin{array}{cc}
-N(s) & 0 \\
0 & N(t)
\end{array}\right)\left(\begin{array}{c}
\lambda(s) \\
\lambda(t)
\end{array}\right) \\
& =\left(\begin{array}{c}
-\mathcal{B}^{T}(s) p(s)-\lambda_{0} L_{u}^{T}(s, x(s), u(s))-N^{T}(s) \lambda(s) \\
\mathcal{B}^{T}(t) p(t)+\lambda_{0} L_{u}^{T}(t, x(t), u(t))+N^{T}(t) \lambda(t)
\end{array}\right) \stackrel{(2.11)}{=} 0,
\end{aligned}
$$

so that condition (3.17) is satisfies.

Conversely, assume that (3.13)-(3.15) and (3.17) hold for a feasible pair $(\tilde{x}(\cdot), \tilde{u}(\cdot))$ with the multipliers $\tilde{\lambda}_{0}$, $\tilde{\gamma}, \tilde{p}(\cdot)$, and $\tilde{\lambda}(\cdot)$. Let $(x(\cdot), u(\cdot))$ and $\lambda_{0}, \gamma, p(\cdot)$, and $\lambda(\cdot)$ be defined by (3.11) and (3.20). Then, as in the proof of the "converse" part of Proposition 3.3, conditions (2.7)-(2.9) are satisfied. It only remains to show that (2.11) holds true. For this, stationarity condition (3.17) yields through (3.9), (3.11), (3.19), and (3.20) that for $t \in[c, b]$ and $s:=a+b-t \in[a, c]$ we have

$$
\begin{aligned}
& 0 \stackrel{(3.17)}{=}\left(\begin{array}{c}
\mathcal{B}^{T}(a+b-t) \tilde{p}_{1}(t) \\
\mathcal{B}^{T}(t) \tilde{p}_{2}(t)
\end{array}\right)+\lambda_{0}\left(\begin{array}{c}
-L_{u}^{T}\left(a+b-t, \tilde{x}_{1}(t),-\tilde{u}_{1}(t)\right) \\
L_{u}^{T}\left(t, \tilde{x}_{2}(t), \tilde{u}_{2}(t)\right)
\end{array}\right)+\left(\begin{array}{c}
-N^{T}(a+b-t) \tilde{\lambda}_{1}(t) \\
N^{T}(t) \tilde{\lambda}_{2}(t)
\end{array}\right) \\
& \stackrel{(3.20),(3.11)}{=}\left(\begin{array}{c}
-\mathcal{B}^{T}(s) p(s)-\lambda_{0} L_{u}^{T}(s, x(s), u(s))-N^{T}(s) \lambda(s) \\
\mathcal{B}^{T}(t) p(t)+\lambda_{0} L_{u}^{T}(t, x(t), u(t))+N^{T}(t) \lambda(t)
\end{array}\right) .
\end{aligned}
$$

Since the first entry of (3.27) is satisfied for all $s=a+b-t \in[a, c]$ and the second entry of equation (3.27) is satisfied for all $t \in[c, b]$, then for all $t \in[a, b]$ the stationarity condition (2.11) holds. The proof is complete.

The following result indicates that our transformation preserves the normality (controllability) for either the strong or the weak Pontryagin principle.

Corollary 3.5 (Normality for strong and weak Pontryagin principle). If the problem (C) is normal at a feasible pair $(x(\cdot), u(\cdot))$ satisfying the strong, respectively, the weak Pontryagin principle, then the problem $(\tilde{\mathrm{C}})$ is normal at the pair $(\tilde{x}(\cdot), \tilde{u}(\cdot))$ defined by (3.10) and which satisfies the strong, respectively, the weak Pontryagin principle. Conversely, if the problem $(\tilde{\mathrm{C}})$ is normal at a feasible pair $(\tilde{x}(\cdot), \tilde{u}(\cdot))$ satisfying the strong, respectively, the weak Pontryagin principle, then the problem $(\mathrm{C})$ is normal at the pair $(x(\cdot), u(\cdot))$ defined by $(3.11)$ and which satisfies the strong, respectively, the weak Pontryagin principle. 
Proof. The results follow respectively from Propositions 3.3 and 3.4, since by (3.12) and (3.20) the multipliers $\lambda_{0}$ and $\tilde{\lambda}_{0}$ are transformed into each other.

Remark 3.6. The equivalence of the normality of problem $(\mathrm{C})$ at a feasible pair $(x(\cdot), u(\cdot))$ satisfying the weak Pontryagin principle with the normality of problem $(\tilde{\mathrm{C}})$ at a corresponding feasible pair $(\tilde{x}(\cdot), \tilde{u}(\cdot))$ obtained through (3.10) can also be proven directly by using property (2.14). Following the definition of the matrices $\mathcal{E}(t)$ and $\mathcal{D}$ in $(2.15)$ with the block partition of $M=\left(M_{a}, M_{b}\right)$, we define the augmented matrices

$$
\begin{aligned}
\tilde{M}_{c} & :=\left(\begin{array}{cc}
I & -I \\
0 & 0
\end{array}\right), \quad \tilde{M}_{b}:=\left(\begin{array}{c}
0 \\
M
\end{array}\right)=\left(\begin{array}{cc}
0 & 0 \\
M_{a} & M_{b}
\end{array}\right), \\
\tilde{\mathcal{D}} & :=\tilde{M}_{c}+\tilde{M}_{b} \tilde{\Phi}(b)=\left(\begin{array}{cc}
I & -I \\
M_{a} \Phi^{-1}(c) & M_{b} \Phi(b) \Phi^{-1}(c)
\end{array}\right), \\
\tilde{\mathcal{E}}(t) & :=\tilde{M}_{b} \tilde{\Phi}(b) \tilde{\Phi}^{-1}(t) \tilde{\mathcal{B}}(t) \tilde{Y}(t) \\
& =\left(\begin{array}{cc}
M_{a} \Phi^{-1}(a+b-t) \mathcal{B}(a+b-t) Y(a+b-t) & \mathcal{E}(t)
\end{array}\right), \quad t \in[c, b], \\
\tilde{\Phi}(t) & =\left(\begin{array}{cc}
\Phi(a+b-t) \Phi^{-1}(c) & 0 \\
0 & \Phi(t) \Phi^{-1}(c)
\end{array}\right), \quad t \in[c, b] .
\end{aligned}
$$

Here the function $\tilde{Y}(\cdot)$ is defined in (3.19), $\tilde{\Phi}(\cdot)$ is the fundamental matrix of the system $\tilde{\Phi}^{\prime}=\tilde{\mathcal{A}}(t) \tilde{\Phi}$ for $t \in[c, b]$ satisfying $\tilde{\Phi}(c)=I$, and the matrix $\tilde{M}$ in (3.19) is partitioned as $\tilde{M}=\left(\tilde{M}_{c}, \tilde{M}_{b}\right)$. Then it can be verified that condition (2.14) holds if and only if

$$
\left\{\tilde{\mathcal{E}}^{T}(t) \tilde{\gamma}=0, \quad t \in[c, b], \quad \tilde{\mathcal{D}}^{T} \tilde{\gamma}=0, \quad \tilde{\gamma} \in \mathbb{R}^{n+r}\right\} \quad \Rightarrow \quad \tilde{\gamma}=0
$$

proving the desired statement.

Next we examine the second variation of problem $(\tilde{\mathrm{C}})$ and the corresponding (strengthened) Legendre-Clebsch condition. In particular, we elaborate on their correspondence in the problems $(\tilde{\mathrm{C}})$ and $(\mathrm{C})$. A pair $(\tilde{\eta}(\cdot), \tilde{v}(\cdot))$ is admissible for problem ( $\tilde{\mathrm{C}})$ if $\tilde{\eta}(\cdot) \in \mathrm{C}_{\mathrm{p}}^{1}\left([c, b], \mathbb{R}^{2 n}\right)$ and $\tilde{v}(\cdot) \in \mathrm{C}_{\mathrm{p}}\left([c, b], \mathbb{R}^{2 m}\right)$ and

$$
\dot{\tilde{\eta}}(t)=\tilde{\mathcal{A}}(t) \tilde{\eta}(t)+\tilde{\mathcal{B}}(t) \tilde{v}(t), \quad \tilde{N}(t) \tilde{v}(t)=0, \quad t \in[c, b], \quad \tilde{M}\left(\begin{array}{l}
\tilde{\eta}(c) \\
\tilde{\eta}(b)
\end{array}\right)=0
$$

where we recall that $\tilde{\mathcal{A}}(t), \tilde{\mathcal{B}}(t), \tilde{N}(t)$, and $\tilde{M}$ are given by (3.19). We will use the partitions $\tilde{\eta}=\left(\tilde{\eta}_{1}^{T}, \tilde{\eta}_{2}^{T}\right)^{T}$ and $\tilde{v}=\left(\tilde{v}_{1}^{T}, \tilde{v}_{2}^{T}\right)^{T}$ in a similar way to $(3.5)$.

Given an admissible pair $(\eta(\cdot), v(\cdot))$ for problem $(\mathrm{C})$, that is, the functions $\eta(\cdot) \in \mathrm{C}_{\mathrm{p}}^{1}\left([a, b], \mathbb{R}^{n}\right)$ and $v(\cdot) \in$ $\mathrm{C}_{\mathrm{p}}\left([a, b], \mathbb{R}^{m}\right)$ satisfy condition $(2.5)$, we define the pair $(\tilde{\eta}(\cdot), \tilde{v}(\cdot))$ on $[c, b]$ by

$$
\tilde{\eta}(t):=\left(\begin{array}{c}
\eta(a+b-t) \\
\eta(t)
\end{array}\right), \quad \tilde{v}(t):=\left(\begin{array}{c}
-v(a+b-t) \\
v(t)
\end{array}\right), \quad t \in[c, b] .
$$

Conversely, given an admissible $(\tilde{\eta}(\cdot), \tilde{v}(\cdot))$ for $(\tilde{\mathrm{C}})$ we define the pair $(\eta(\cdot), v(\cdot))$ on $[a, b]$ by

$$
\eta(t):=\left\{\begin{array}{ll}
\tilde{\eta}_{1}(a+b-t), & t \in[a, c], \\
\tilde{\eta}_{2}(t), & t \in[c, b],
\end{array} \quad v(t):= \begin{cases}-\tilde{v}_{1}(a+b-t), & t \in[a, c], \\
\tilde{v}_{2}(t), & t \in[c, b] .\end{cases}\right.
$$

Formulas (3.30) and (3.31) are compatible with the corresponding definitions in (3.10) and (3.11). 
Assume that problem $(\tilde{\mathrm{C}})$ is normal at a feasible pair $(\tilde{x}(\cdot), \tilde{u}(\cdot))$ satisfying with its associated multipliers $\tilde{\lambda}_{0}=1, \tilde{\gamma}, \tilde{p}(\cdot)$, and $\tilde{\lambda}(\cdot)$ the weak Pontryagin principle, namely, conditions (3.13)-(3.15) and (3.17) hold. The second variation of the functional $\tilde{J}$ is the quadratic functional

$$
\tilde{J}^{\prime \prime}(\eta, v):=\frac{1}{2}\left(\begin{array}{l}
\tilde{\eta}(c) \\
\tilde{\eta}(b)
\end{array}\right)^{T} \tilde{\Gamma}\left(\begin{array}{l}
\tilde{\eta}(c) \\
\tilde{\eta}(b)
\end{array}\right)+\frac{1}{2} \int_{c}^{b}\left(\begin{array}{l}
\tilde{\eta}(t) \\
\tilde{v}(t)
\end{array}\right)^{T} \nabla_{(\tilde{x}, \tilde{u})}^{2} \tilde{H}(t)\left(\begin{array}{l}
\tilde{\eta}(t) \\
\tilde{v}(t)
\end{array}\right) \mathrm{d} t
$$

where $(\tilde{\eta}(\cdot), \tilde{v}(\cdot))$ is admissible for $(\tilde{\mathrm{C}})$, the matrix $\tilde{\Gamma} \in \mathbb{R}^{4 n \times 4 n}$ is defined by

$$
\tilde{\Gamma}:=\nabla^{2} \tilde{K}(\tilde{x}(c), \tilde{x}(b))+\tilde{\gamma}^{T} \nabla^{2} \tilde{\varphi}(\tilde{x}(c), \tilde{x}(b))=\left(\begin{array}{cc}
0 & 0 \\
0 & \Gamma
\end{array}\right)
$$

with $\Gamma$ given in $(2.16)$, and

$$
\nabla_{(\tilde{x}, \tilde{u})}^{2} \tilde{H}(t)=\left(\begin{array}{cc}
\tilde{P}(t) & \tilde{Q}(t) \\
\tilde{Q}^{T}(t) & \tilde{R}(t)
\end{array}\right):=\left(\begin{array}{cc}
\tilde{H}_{\tilde{x} \tilde{x}}(t) & \tilde{H}_{\tilde{x} \tilde{u}}(t) \\
\tilde{H}_{\tilde{u} \tilde{x}}(t) & \tilde{H}_{\tilde{u} \tilde{u}}(t)
\end{array}\right)
$$

Here the Hamiltonian $\tilde{H}$ is defined in (3.7) and its second order partial derivatives in (3.33) are evaluated at the argument $(t, \tilde{x}(t), \tilde{u}(t), \tilde{p}(t), \tilde{\lambda}(t), 1)$. Clearly, we know that the functions $\tilde{P}(\cdot) \in \mathrm{C}_{\mathrm{p}}\left([c, b], \mathbb{R}^{2 n \times 2 n}\right), \tilde{Q}(\cdot) \in$ $\mathrm{C}_{\mathrm{p}}\left([c, b], \mathbb{R}^{2 n \times 2 m}\right), \tilde{R}(\cdot) \in \mathrm{C}_{\mathrm{p}}\left([c, b], \mathbb{R}^{2 m \times 2 m}\right)$, and from (3.9) we obtain that for $t \in[c, b]$ we have

$$
\left.\begin{array}{c}
\tilde{P}(t)=\left(\begin{array}{cc}
P(a+b-t) & 0 \\
0 & P(t)
\end{array}\right), \quad \tilde{Q}(t)=\left(\begin{array}{cc}
-Q(a+b-t) & 0 \\
0 & Q(t)
\end{array}\right), \\
\tilde{R}(t)=\left(\begin{array}{cc}
R(a+b-t) & 0 \\
0 & R(t)
\end{array}\right) .
\end{array}\right\}
$$

Recall that the functional $\tilde{J}^{\prime \prime}$ is nonnegative if $\tilde{J}^{\prime \prime}(\tilde{\eta}, \tilde{v}) \geq 0$ for all admissible pairs $(\tilde{\eta}(\cdot), \tilde{v}(\cdot))$ for problem $(\tilde{\mathrm{C}})$, and the functional $\tilde{J}^{\prime \prime}$ is coercive if there exists $\tilde{\alpha}>0$ such that

$$
\tilde{J}^{\prime \prime}(\tilde{\eta}, \tilde{v}) \geq \frac{\tilde{\alpha}}{2}\left\{|\tilde{\eta}(c)|^{2}+|\tilde{\eta}(b)|^{2}+\int_{c}^{b}\left\{|\tilde{\eta}(t)|^{2}+|\tilde{v}(t)|^{2}\right\} \mathrm{d} t\right\}
$$

for all admissible pairs $(\tilde{\eta}(\cdot), \tilde{v}(\cdot))$ for $(\tilde{\mathrm{C}})$.

Proposition 3.7 (Admissibility). If a pair $(\eta(\cdot), v(\cdot))$ is admissible for $(\mathrm{C})$, then the pair $(\tilde{\eta}(\cdot), \tilde{v}(\cdot))$ defined by (3.30) is admissible for $(\tilde{\mathrm{C}})$ with the objective value $\tilde{J}^{\prime \prime}(\tilde{\eta}, \tilde{v})=J^{\prime \prime}(\eta, v)$. Conversely, if a pair $(\tilde{\eta}(\cdot), \tilde{v}(\cdot))$ is admissible for $(\tilde{\mathrm{C}})$, then the pair $(\eta(\cdot), v(\cdot))$ defined by $(3.31)$ is admissible for $(\mathrm{C})$ with the objective value $J^{\prime \prime}(\eta, v)=\tilde{J}^{\prime \prime}(\tilde{\eta}, \tilde{v})$.

Proof. The statement follows by applying Proposition 3.1 to the second variation $J^{\prime \prime}$ over the constraints $(2.5)$ and to the second variation $\tilde{J}^{\prime \prime}$ over the constraints (3.29).

Proposition 3.8 (Second variation). The second variation $J^{\prime \prime}$ is coercive (nonnegative) if and only if the second variation $\tilde{J}^{\prime \prime}$ is coercive (nonnegative).

Proof. The statement about the nonnegativity follows directly from Proposition 3.7, since the values of $J^{\prime \prime}(\eta, v)$ and $\tilde{J}^{\prime \prime}(\tilde{\eta}, \tilde{v})$ are the same under the definitions in (3.30) and (3.31). The coercivity requires some additional analysis. Assume that the second variation $J^{\prime \prime}$ is coercive with the constant $\alpha>0$, i.e., (2.18) holds. Let $(\tilde{\eta}(\cdot), \tilde{v}(\cdot))$ 
be admissible for $(\tilde{\mathrm{C}})$. Then we know from Proposition 3.7 that the corresponding admissible $(\eta(\cdot), v(\cdot))$ defined in (3.31) satisfies

$$
\begin{aligned}
\tilde{J}^{\prime \prime}(\tilde{\eta}, \tilde{v}) & =J^{\prime \prime}(\eta, v) \geq \frac{\alpha}{2}\left\{|\eta(a)|^{2}+|\eta(b)|^{2}+\int_{a}^{b}\left\{|\eta(t)|^{2}+|v(t)|^{2}\right\} \mathrm{d} t\right\} \\
& =\frac{\alpha}{2}\left\{|\tilde{\eta}(b)|^{2}+\int_{c}^{b}\left\{|\tilde{\eta}(t)|^{2}+|\tilde{v}(t)|^{2}\right\} \mathrm{d} t\right\},
\end{aligned}
$$

where in the last step the integral over $[a, b]$ is separated into two integrals over $[a, c]$ and $[c, b]$ and the integral over $[a, c]$ is transformed by $s:=a+b-t$ to an integral over $[c, b]$. Now, by using the fundamental matrix $\tilde{\Phi}(t)$ from (3.28) we have

$$
\tilde{\eta}(t)=\tilde{\Phi}(t) \tilde{\eta}(c)+\tilde{\Phi}(t) \int_{c}^{t} \tilde{\Phi}^{-1}(\tau) \tilde{\mathcal{B}}(\tau) \tilde{v}(\tau) \mathrm{d} \tau, \quad t \in[c, b]
$$

Taking $t=b$ in (3.36) and using the boundedness of $\tilde{\Phi}^{-1}(\cdot)$ and $\tilde{\mathcal{B}}(\cdot)$, it follows that for some constant $\beta>0$ we have

$$
|\tilde{\eta}(c)| \leq \beta\left\{|\tilde{\eta}(b)|+\int_{c}^{b}|\tilde{v}(\tau)| \mathrm{d} \tau\right\} \leq \beta\left\{|\tilde{\eta}(b)|+(b-c)^{\frac{1}{2}}\left(\int_{c}^{b}|\tilde{v}(\tau)|^{2} \mathrm{~d} \tau\right)^{\frac{1}{2}}\right\},
$$

where the last inequality follows from the Cauchy-Schwarz inequality. It results that for some constant $\kappa>0$ we have

$$
\begin{aligned}
|\tilde{\eta}(c)|^{2} & \leq \frac{\kappa}{2}\left\{|\tilde{\eta}(b)|^{2}+\int_{c}^{b}|\tilde{v}(\tau)|^{2} \mathrm{~d} \tau+2|\tilde{\eta}(b)|\left(\int_{c}^{b}|\tilde{v}(\tau)|^{2} \mathrm{~d} \tau\right)^{\frac{1}{2}}\right\} \\
& \leq \kappa\left(|\tilde{\eta}(b)|^{2}+\int_{c}^{b}|\tilde{v}(\tau)|^{2} \mathrm{~d} \tau\right)
\end{aligned}
$$

where the last inequality is obtained from the arithmetic-geometric mean inequality (i.e., from $2 x y \leq x^{2}+y^{2}$ ). Set $\tilde{\alpha}:=\min \left\{\frac{\alpha}{2}, \frac{\alpha}{2 \kappa}\right\}$ and use (3.37) in (3.35) we obtain

$$
\begin{aligned}
\tilde{J}^{\prime \prime}(\tilde{\eta}, \tilde{v}) & \geq \frac{\alpha}{4}\left\{|\tilde{\eta}(b)|^{2}+\int_{c}^{b}\left\{|\tilde{\eta}(t)|^{2}+|\tilde{v}(t)|^{2}\right\} \mathrm{d} t\right\}+\frac{\alpha}{4 \kappa}|\tilde{\eta}(c)|^{2} \\
& \geq \frac{\tilde{\alpha}}{2}\left\{|\tilde{\eta}(c)|^{2}+|\tilde{\eta}(b)|^{2}+\int_{c}^{b}\left\{|\tilde{\eta}(t)|^{2}+|\tilde{v}(t)|^{2}\right\} \mathrm{d} t\right\},
\end{aligned}
$$

which shows that the functional $\tilde{J}^{\prime \prime}$ is coercive.

Conversely, assume that $\tilde{J}^{\prime \prime}$ is coercive with the constant $\tilde{\alpha}>0$. Let $(\eta(\cdot), v(\cdot))$ be an admissible pair for (C). Then by Proposition 3.7 we know that that the corresponding admissible $(\tilde{\eta}(\cdot), \tilde{v}(\cdot))$ defined in $(3.30)$ is admissible for $(\tilde{\mathrm{C}})$, and hence it satisfies

$$
\begin{aligned}
J^{\prime \prime}(\eta, v) & =\tilde{J}^{\prime \prime}(\tilde{\eta}, \tilde{v}) \geq \frac{\tilde{\alpha}}{2}\left\{|\tilde{\eta}(c)|^{2}+|\tilde{\eta}(b)|^{2}+\int_{c}^{b}\left\{|\tilde{\eta}(t)|^{2}+|\tilde{v}(t)|^{2}\right\} \mathrm{d} t\right\} \\
\stackrel{(3.30)}{=} & \frac{\tilde{\alpha}}{2}\left\{2|\eta(c)|^{2}+|\eta(a)|^{2}+|\eta(b)|^{2}+\int_{c}^{b}\left\{|\eta(a+b-t)|^{2}+|v(a+b-t)|^{2}\right\} \mathrm{d} t\right.
\end{aligned}
$$




$$
\begin{aligned}
& \left.+\int_{c}^{b}\left\{|\eta(t)|^{2}+|v(t)|^{2}\right\} \mathrm{d} t\right\} \\
\geq & \frac{\tilde{\alpha}}{2}\left\{|\eta(a)|^{2}+|\eta(b)|^{2}+\int_{a}^{b}\left\{|\eta(t)|^{2}+|v(t)|^{2}\right\} \mathrm{d} t\right\},
\end{aligned}
$$

where the last inequality is obtained by disregarding the nonnegative term $2|\eta(c)|^{2}$ and by substituting $s:=$ $a+b-t$ in the first integral of the preceding expression. Therefore, the functional $J^{\prime \prime}$ is coercive with the constant $\alpha:=\tilde{\alpha}$. The proof is complete.

According to (2.19) and (2.20), the Legendre-Clebsch condition is defined in problem ( $\tilde{\mathrm{C}})$ as

$$
\tilde{\mathcal{Y}}^{T}(t) \tilde{R}(t) \tilde{\mathcal{Y}}(t) \geq 0, \quad t \in[c, b]
$$

and the strengthened Legendre-Clebsch condition for problem $(\tilde{\mathrm{C}})$ is

$$
\tilde{\mathcal{Y}}^{T}(t) \tilde{R}(t) \tilde{\mathcal{Y}}(t)>0, \quad t \in[c, b],
$$

where $\tilde{\mathcal{Y}}(\cdot) \in \mathrm{C}_{\mathrm{p}}\left([c, b], \mathbb{R}^{2 m \times 2(m-k)}\right)$ is a matrix function for which the columns of $\tilde{\mathcal{Y}}(t)$ form an orthonormal basis for the kernel of $\tilde{N}(t)$ on $[c, b]$, i.e., $\tilde{N}(t) \tilde{\mathcal{Y}}(t)=0$ and $\tilde{\mathcal{Y}}^{T}(t) \tilde{\mathcal{Y}}(t)=I_{2(m-k)}$ on $[c, b]$.

Remark 3.9. The matrix function $\tilde{\mathcal{Y}}(\cdot)$ in $(3.38)$ and (3.39) can be taken to be the block diagonal matrix function $\tilde{Y}(\cdot)$ defined in (3.19). The reason for this is that the columns of each of the matrices $\tilde{\mathcal{Y}}(t)$ and $\tilde{Y}(t)$ form an orthonormal basis for the kernel of $\tilde{N}(t)$ on $[c, b]$, and hence $\tilde{\mathcal{Y}}(t)=\tilde{Y}(t) Z(t)$ for all $t \in[c, b]$, where $Z(t)$ is an orthogonal $2(m-k) \times 2(m-k)$ matrix. Since $Y^{T}(t) \tilde{Y}(t)=I_{2(m-k)}$ on $[c, b]$, then the matrix $Z(t)$ is unique and it is given by the formula $Z(t)=\tilde{Y}^{T}(t) \tilde{\mathcal{Y}}(t)$ on $[c, b]$ and thus, $Z(\cdot) \in \mathrm{C}_{\mathrm{p}}\left([c, b], \mathbb{R}^{2(m-k) \times 2(m-k)}\right)$.

Next we show the equivalence of the (strengthened) Legendre-Clebsch conditions for problems $(\mathrm{C})$ and $(\tilde{\mathrm{C}})$. The correspondence between the quantities $(x(\cdot), u(\cdot), p(\cdot), \lambda(\cdot))$ in problem $(\mathrm{C})$ and $(\tilde{x}(\cdot), \tilde{u}(\cdot), \tilde{p}(\cdot), \tilde{\lambda}(\cdot))$ in problem ( $\tilde{\mathrm{C}})$ is determined by equations (3.10), (3.12) and (3.11), (3.20).

Proposition 3.10 (Legendre-Clebsch condition). The Legendre-Clebsch condition (2.19) is satisfied at $(x(\cdot), u(\cdot), p(\cdot), \lambda(\cdot))$ in problem (C) if and only if the Legendre-Clebsch condition (3.38), or equivalently

$$
\tilde{Y}^{T}(t) \tilde{R}(t) \tilde{Y}(t) \geq 0, \quad t \in[c, b],
$$

is satisfied at $(\tilde{x}(\cdot), \tilde{u}(\cdot), \tilde{p}(\cdot), \tilde{\lambda}(\cdot))$ in problem $(\tilde{\mathrm{C}})$, where the matrix functions $\tilde{R}(\cdot)$ and $\tilde{Y}(\cdot)$ are given in (3.34) and (3.19). The strengthened Legendre-Clebsch condition $(2.20)$ is satisfied at $(x(\cdot), u(\cdot), p(\cdot), \lambda(\cdot))$ in problem (C) if and only if the strengthened Legendre-Clebsch condition (3.39), or equivalently

$$
\tilde{Y}^{T}(t) \tilde{R}(t) \tilde{Y}(t)>0, \quad t \in[c, b],
$$

is satisfied at $(\tilde{x}(\cdot), \tilde{u}(\cdot), \tilde{p}(\cdot), \tilde{\lambda}(\cdot))$ in problem $(\tilde{\mathrm{C}})$.

Proof. By splitting the Legendre-Clebsch condition (2.19) in problem (C) onto the subintervals $[a, c]$ and $[c, b]$, it follows that (2.19) is equivalent with

$$
Y^{T}(a+b-t) R(a+b-t) Y(a+b-t) \geq 0, \quad Y^{T}(t) R(t) Y(t) \geq 0, \quad t \in[c, b] .
$$

In turn, by using the form of $\tilde{R}(t)$ and $\tilde{Y}(t)$ in (3.34) and (3.19) we can see that (3.42) is equivalent to (3.40). The equivalence of (3.40) and (3.38) follows from Remark 3.9, which completes the proof of the first statement. 
The equivalence of the strengthened Legendre-Clebsch conditions (2.20), (3.41), and (3.39) follows by the same arguments with the strict inequalities in (3.42).

Remark 3.11. We emphasize that in addition to preserving the normality of the considered optimal control problems (Corollary 3.5), the transformation presented in this section also preserves the structure of the calculus of variations problems. More specifically, if $(\mathrm{C})$ is a calculus of variations problem, i.e., if the functions defining constraints (2.1) and (2.2) have the form $f(t, x, u)=u$ and $\psi(t, u) \equiv 0$, then we have $u(t)=\dot{x}(t)$ in the objective functional $J$ and $\mathcal{A}(t) \equiv 0, \mathcal{B}(t) \equiv I$ (with $m=n$ ), and $N(t) \equiv 0$ (with $k=0$ ). Whence, by (3.4), the constraints (3.1) and (3.2) of the transformed problem ( $\tilde{\mathrm{C}})$ have the same form as those of $(\mathrm{C})$, that is, $\tilde{f}(t, \tilde{x}, \tilde{u})=\tilde{u}$ and $\tilde{\psi}(t, \tilde{u}) \equiv 0$. This means that $\tilde{u}(t)=\dot{\tilde{x}}(t)$ holds on $[c, b]$ in the objective functional $\tilde{J}$, and $\tilde{\mathcal{A}}(t) \equiv 0, \tilde{\mathcal{B}}(t) \equiv I$, and $\tilde{N}(t) \equiv 0$ by $(3.19)$.

Next we comment about the preservation of the controllability (normality) of the Jacobi systems for problems $(\mathrm{C})$ and $(\tilde{\mathrm{C}})$.

Remark 3.12. For the transformed problem $(\tilde{\mathrm{C}})$ and a normal feasible pair $(\tilde{x}(\cdot), \tilde{u}(\cdot))$ we associate the augmented Jacobi system (or augmented linear Hamiltonian system)

$$
\dot{\tilde{\eta}}=\tilde{A}(t) \tilde{\eta}+\tilde{B}(t) \tilde{\xi}, \quad \dot{\tilde{\xi}}=\tilde{C}(t) \tilde{\eta}-\tilde{A}^{T}(t) \tilde{\xi}, \quad t \in[c, b]
$$

where $\tilde{\eta}(\cdot), \tilde{\xi}(\cdot) \in \mathrm{C}_{\mathrm{p}}^{1}\left([c, b], \mathbb{R}^{2 n}\right)$ and the coefficients $\tilde{A}(\cdot), \tilde{B}(\cdot), \tilde{C}(\cdot) \in \mathrm{C}_{\mathrm{p}}\left([c, b], \mathbb{R}^{2 n \times 2 n}\right)$ are given on the interval $[c, b]$ by the formulas

$$
\left.\begin{array}{c}
\tilde{A}(t)=\left(\begin{array}{cc}
-A(a+b-t) & 0 \\
0 & A(t)
\end{array}\right), \quad \tilde{B}(t)=\left(\begin{array}{cc}
B(a+b-t) & 0 \\
0 & B(t)
\end{array}\right), \\
\tilde{C}(t)=\left(\begin{array}{cc}
C(a+b-t) & 0 \\
0 & C(t)
\end{array}\right)
\end{array}\right\}
$$

with $A(t), B(t), C(t)$ defined in (2.22). According to equations (3.10), (3.12) and (3.11), (3.20), the solutions $(\eta(\cdot), \xi(\cdot))$ of $(2.21)$ and $(\tilde{\eta}(\cdot), \tilde{\xi}(\cdot))$ of $(3.43)$ are related by formulas

$$
\tilde{\eta}(t):=\left(\begin{array}{c}
\eta(a+b-t) \\
\eta(t)
\end{array}\right), \quad \tilde{\xi}(t):=\left(\begin{array}{c}
\xi(a+b-t) \\
-\xi(t)
\end{array}\right), \quad t \in[c, b]
$$

and

$$
\eta(t):=\left\{\begin{array}{ll}
\tilde{\eta}_{1}(a+b-t), & t \in[a, c], \\
\tilde{\eta}_{2}(t), & t \in[c, b],
\end{array} \quad \xi(t):= \begin{cases}\tilde{\xi}_{1}(a+b-t), & t \in[a, c], \\
-\tilde{\xi}_{2}(t), & t \in[c, b] .\end{cases}\right.
$$

By Corollary 3.5 we know that the normality of problems $(\mathrm{C})$ and $(\tilde{\mathrm{C}})$ is preserved, which is in view of Remark 2.10 carried over to the Jacobi systems (2.21) and (3.43). The equivalence of the normality for systems (2.21) and (3.43) can also be proven directly by using the structure of the pairs $(\tilde{\eta}(\cdot), \tilde{\xi}(\cdot))$ and $(\eta(\cdot), \xi(\cdot))$ in (3.45) and (3.46). Note also that, due to the form of the matrix $\tilde{B}(t)$ in (3.44), the corresponding Legendre conditions $B(t) \geq 0$ on $[a, b]$ and $\tilde{B}(t) \geq 0$ on $[c, b]$ are also preserved. This is essential e.g. for the conjugate and coupled point theory for systems (2.21) and (3.43).

In the last part of this section we discuss the transformation of problem $(\mathrm{C})$, which is announced in page 886 of [36] in the context of the Jacobi system (2.21), and compare it with the transformation presented above. We note that the outcome of this transformation was essentially used in Theorem 2.1 of [1] for deriving an extended Riccati type sufficient condition for a periodic nonlinear optimal control problem. In the latter reference, the 
authors use a cascade system of three differential equations, which consists of the Riccati matrix equation associated with the Jacobi system (2.21) together with a linear differential equation and an integrator. This cascade system is equivalent to the augmented Riccati matrix equation associated with the corresponding Jacobi system (3.54) displayed below.

Remark 3.13. Together with the basic problem (C) we consider the augmented nonlinear optimal control problem over the interval $[a, b]$ with separated endpoints

$$
\operatorname{minimize} \quad \bar{J}(\bar{x}, \bar{u}):=\bar{K}_{a}(\bar{x}(a))+\bar{K}_{b}(\bar{x}(b))+\int_{a}^{b} \bar{L}(t, \bar{x}(t), \bar{u}(t)) \mathrm{d} t
$$

subject to $\bar{x}(\cdot) \in \mathrm{C}_{\mathrm{p}}^{1}\left([a, b], \mathbb{R}^{2 n}\right)$ and $\bar{u}(\cdot) \in \mathrm{C}_{\mathrm{p}}\left([a, b], \mathbb{R}^{m}\right)$ such that

$$
\dot{\bar{x}}(t)=\bar{f}(t, \bar{x}(t), \bar{u}(t)), \quad \bar{\psi}(t, \bar{u}(t))=0, \quad t \in[a, b], \quad \bar{\varphi}_{a}(\bar{x}(a))=0, \quad \bar{\varphi}_{b}(\bar{x}(b))=0 .
$$

Note that the state $\bar{x}(\cdot)$ is augmented into double dimension $2 n$, while the control $\bar{u}(\cdot)$ remains in the original dimension $m$. Given the data $(L, f, \psi, K, \varphi)$ of problem $(\mathrm{C})$ we define the corresponding data of problem $(\overline{\mathrm{C}})$ by the functions

$$
\begin{array}{lll}
\bar{L}:[a, b] \times \mathbb{R}^{2 n} \times \mathbb{R}^{m} \rightarrow \mathbb{R}, & \bar{K}_{c}: \mathbb{R}^{2 n} \rightarrow \mathbb{R}, & \bar{K}_{b}: \mathbb{R}^{2 n} \rightarrow \mathbb{R}, \\
\bar{f}:[a, b] \times \mathbb{R}^{2 n} \times \mathbb{R}^{m} \rightarrow \mathbb{R}^{2 n}, & \bar{\varphi}_{a}: \mathbb{R}^{2 n} \rightarrow \mathbb{R}^{n}, & \bar{\varphi}_{b}: \mathbb{R}^{2 n} \rightarrow \mathbb{R}^{r}, \\
\bar{\psi}:[a, b] \times \mathbb{R}^{m} \rightarrow \mathbb{R}^{k}, &
\end{array}
$$

where

$$
\left.\begin{array}{c}
\bar{L}(t, \bar{x}, \bar{u}):=L\left(t, \bar{x}_{2}, \bar{u}\right), \quad \bar{f}(t, \bar{x}, \bar{u}):=\left(\begin{array}{c}
0 \\
f\left(t, \bar{x}_{2}, \bar{u}\right)
\end{array}\right), \quad \bar{\psi}(t, \bar{u}):=\psi(t, \bar{u}), \\
\bar{K}_{a}(\bar{x}):=0 \quad \bar{K}_{b}(\bar{x}):=K\left(\bar{x}_{1}, \bar{x}_{2}\right), \quad \bar{\varphi}_{a}(\bar{x}):=\bar{x}_{1}-\bar{x}_{2}, \quad \bar{\varphi}_{b}(\bar{x}):=\varphi\left(\bar{x}_{1}, \bar{x}_{2}\right) .
\end{array}\right\}
$$

In the spirit of $(3.10)$ and $(3.11)$ the feasible pairs $(\bar{x}(\cdot), \bar{u}(\cdot))$ for problem $(\overline{\mathrm{C}})$ and $(x(\cdot), u(\cdot))$ for problem $(\mathrm{C})$ are related by the formulas

$$
\bar{x}(t):=\left(\begin{array}{c}
x(a) \\
x(t)
\end{array}\right), \quad \bar{u}(t):=u(t), \quad t \in[a, b],
$$

and

$$
x(t):=\tilde{x}_{2}(t), \quad u(t):=\bar{u}(t), \quad t \in[a, b] .
$$

It follows that the feasibility and the (strict) strong/weak local minimality of the pairs $(x(\cdot), u(\cdot))$ for problem $(\mathrm{C})$ and $(\bar{x}(\cdot), \bar{u}(\cdot))$ for problem $(\overline{\mathrm{C}})$ connected by equations (3.48) and (3.49) are preserved, including the objective value $J(x, u)=\bar{J}(\bar{x}, \bar{u})$. Moreover, the strong/weak Pontryagin principle for problems $(\mathrm{C})$ and $(\overline{\mathrm{C}})$ according to Definitions 2.1 and 2.2 are preserved as well with the matrices $\bar{N}(t)=N(t), \bar{Y}(t)=Y(t)$, and

$$
\overline{\mathcal{A}}(t)=\left(\begin{array}{cc}
0 & 0 \\
0 & \mathcal{A}(t)
\end{array}\right), \quad \overline{\mathcal{B}}(t)=\left(\begin{array}{c}
0 \\
\mathcal{B}(t)
\end{array}\right), \quad \bar{M}=\left(\begin{array}{ccc}
I & -I & 0 \\
0 & 0 & M
\end{array}\right)
$$


defined on $[a, b]$ and with the corresponding multipliers given by

$$
\bar{\lambda}_{0}:=\lambda_{0}, \quad \bar{\gamma}:=\left(\begin{array}{c}
-p(a) \\
\gamma
\end{array}\right), \quad \bar{p}(t):=\left(\begin{array}{c}
-p(a) \\
p(t)
\end{array}\right), \quad \bar{\lambda}(t):=\lambda(t), \quad t \in[a, b],
$$

and by

$$
\lambda_{0}:=\bar{\lambda}_{0}, \quad \gamma:=\bar{\gamma}_{2}, \quad p(t):=\bar{p}_{2}(t), \quad \lambda(t):=\bar{\lambda}(t), \quad t \in[a, b] .
$$

Note that if problem $(\mathrm{C})$ is a calculus of variations problem (i.e., $m=n, f(t, x, u)=u$, and $\psi(t, u)=0)$, then the corresponding augmented problem $(\overline{\mathrm{C}})$ does not preserve this structure. The coefficients of the associated second variation $\bar{J}^{\prime \prime}(\bar{\eta}, \bar{v})$ of problem $(\overline{\mathrm{C}})$ at a feasible pair $(\bar{x}(\cdot), \bar{u}(\cdot))$ are according to $(2.17)$ given by

$$
\bar{P}(t)=\left(\begin{array}{cc}
0 & 0 \\
0 & P(t)
\end{array}\right), \quad \bar{Q}(t)=\left(\begin{array}{c}
0 \\
Q(t)
\end{array}\right), \quad \bar{R}(t)=R(t), \quad t \in[a, b] .
$$

Finally, the corresponding augmented Jacobi system has the form

$$
\dot{\bar{\eta}}=\bar{A}(t) \bar{\eta}+\bar{B}(t) \bar{\xi}, \quad \dot{\bar{\xi}}=\bar{C}(t) \bar{\eta}-\bar{A}^{T}(t) \bar{\xi}, \quad t \in[a, b]
$$

where $\bar{\eta}(\cdot), \bar{\xi}(\cdot) \in \mathrm{C}_{\mathrm{p}}^{1}\left([a, b], \mathbb{R}^{2 n}\right)$ and where the coefficients $\bar{A}(\cdot), \bar{B}(\cdot), \bar{C}(\cdot) \in \mathrm{C}_{\mathrm{p}}\left([a, b], \mathbb{R}^{2 n \times 2 n}\right)$ are given, in view of $(2.22)$ and (3.53), by the formulas

$$
\bar{A}(t)=\left(\begin{array}{cc}
0 & 0 \\
0 & A(t)
\end{array}\right), \quad \bar{B}(t)=\left(\begin{array}{cc}
0 & 0 \\
0 & B(t)
\end{array}\right), \quad \bar{C}(t)=\left(\begin{array}{cc}
0 & 0 \\
0 & C(t)
\end{array}\right)
$$

with $A(t), B(t), C(t)$ defined in (2.22). Therefore, we conclude that the transformation of problem (C) into augmented problem $(\overline{\mathrm{C}})$ enjoys the same properties (regarding the feasibility, the strong/weak local optimality, the strong/weak Pontryagin principle, the normality of these problems, including the normality of their Jacobi systems, as well as the nonnegativity and coercivity of the second variation and the validity of the Legendre condition) as the transformation into augmented problem $(\tilde{\mathrm{C}})$, with the exception that problem $(\overline{\mathrm{C}})$ is not suitable when the calculus of variations structure is required to be preserved.

Remark 3.14. Following the preceding remark, the transformation into augmented problem $(\overline{\mathrm{C}})$ is suitable for nonlinear optimal control problems on time scales, such as in $[6,7,17,31,33]$. In this context the sensitivity result in Theorem 4.3 of [33] extends (in the spirit of Thm. 4.1 below) to nonlinear optimal control problems on time scales with jointly varying endpoints.

\section{Application of transformation to SEnsitivity AnAlysis}

As an application of the results established in Section 3 we present a generalization to the jointly varying state endpoints constraints of the sensitivity analysis result known in Theorem 4.3 of [33] for nonlinear optimal control problems with separated endpoints. Consider the following parametric nonlinear optimal control problem associated with problem (C) of Section 2

$$
\text { minimize } \quad J(x, u, \omega):=K(x(a), x(b), \omega)+\int_{a}^{b} L(t, x(t), u(t), \omega) \mathrm{d} t
$$

subject to $x(\cdot) \in \mathrm{C}_{\mathrm{p}}^{1}\left([a, b], \mathbb{R}^{n}\right)$ and $u(\cdot) \in \mathrm{C}_{\mathrm{p}}\left([a, b], \mathbb{R}^{m}\right)$ such that

$$
\dot{x}(t)=f(t, x(t), u(t), \omega), \quad \psi(t, u(t), \omega)=0, \quad t \in[a, b], \quad \varphi(x(a), x(b), \omega)=0 .
$$


Here the data of problem $\left(\mathrm{C}_{\omega}\right)$ are similar to those of problem $(\mathrm{C})$ with the special distinction that they now depend also on the parameter $\omega \in \mathbb{R}^{d}$. The Hamiltonian corresponding to problem $\left(\mathrm{C}_{\omega}\right)$, when it is normal, is defined by

$$
H(t, x, u, p, \lambda, \omega):=p^{T} f(t, x, u, \omega)+L(t, x, u, \omega)+\lambda^{T} \psi(t, u, \omega) .
$$

For the remaining part of this section we fix a value $\hat{\omega} \in \mathbb{R}^{d}$ and a pair $(\hat{x}(\cdot), \hat{u}(\cdot))$ feasible for problem $\left(\mathrm{C}_{\hat{\omega}}\right)$. We will assume the following hypothesis $(\hat{\mathrm{A}} 2)$ to hold on the data near $((\hat{x}(\cdot), \hat{u}(\cdot), \hat{\omega})$, where we set $h(t, x, u, \omega):=(L(t, x, u, \omega), f(t, x, u, \omega), \psi(t, u, \omega))$.

(Â2) There exists $\delta_{1}>0$ such that for each $t \in[a, b]$ the function $h(t, \cdot, \cdot, \cdot)$ is twice differentiable on $B_{\delta_{1}}(\hat{x}(t), \hat{u}(t)) \times B_{\delta_{1}}(\hat{\omega})$ and the functions $h, \nabla_{(x, u)} h$, and $\nabla_{(x, u)}^{2} h$ are continuous; the functions $K(\cdot, \cdot, \cdot)$ and $\varphi(\cdot, \cdot, \cdot)$ are twice continuously differentiable on $B_{\delta_{1}}(\hat{x}(a), \hat{x}(b), \hat{\omega})$ and the matrices $\hat{M}:=\nabla \varphi(\hat{x}(a), \hat{x}(b), \hat{\omega})$ and $\hat{N}(t):=\psi_{u}(t, \hat{u}(t), \hat{\omega})$ for $t \in[a, b]$ have full rank.

All the notations used earlier for problem $(\mathrm{C})$ carry over for problem $\left(\mathrm{C}_{\omega}\right)$, and the "hat" placed over the functions indicates the evaluations at the "hat" functions. For instance, $\hat{Y}(\cdot)$ denotes the matrix function in $\mathrm{C}_{\mathrm{p}}\left([a, b], \mathbb{R}^{m \times(m-k)}\right)$ such that for all $t \in[a, b]$ the columns of $\hat{Y}(t)$ form an orthonormal basis for the kernel of $\hat{N}(t)$, and

$$
\begin{gathered}
\hat{\mathcal{A}}(t):=f_{x}(t, \hat{x}(t), \hat{u}(t), \hat{\omega}), \quad \hat{\mathcal{B}}(t):=f_{u}(t, \hat{x}(t), \hat{u}(t), \hat{\omega}), \\
\hat{H}(t):=H(t, \hat{x}(t), \hat{u}(t), \hat{p}(t), \hat{\lambda}(t), \hat{\omega}), \\
\nabla_{(x, u)}^{2} \hat{H}(t)=\left(\begin{array}{cc}
\hat{P}(t) & \hat{Q}(t) \\
\hat{Q}^{T}(t) & \hat{R}(t)
\end{array}\right):=\left(\begin{array}{cc}
\hat{H}_{x x}(t) & \hat{H}_{x u}(t) \\
\hat{H}_{u x}(t) & \hat{H}_{u u}(t)
\end{array}\right) .
\end{gathered}
$$

Now, we are in a position to extend the result in Theorem 4.3 of [33], specialized to the continuous time setting, from the separated state endpoints to the jointly varying state endpoints.

Theorem 4.1 (Sensitivity analysis). Let the assumption (Â2) hold at $(\hat{x}(\cdot), \hat{u}(\cdot), \hat{\omega})$ and the problem $\left(\mathrm{C}_{\hat{\omega}}\right)$ be normal at the feasible pair $(\hat{x}(\cdot), \hat{u}(\cdot))$ satisfying the weak Pontryagin principle. Let $\left(\lambda_{0}=1, \hat{\gamma}, \hat{p}(\cdot), \hat{\lambda}(\cdot)\right)$ be the corresponding multipliers from Definition 2.2 such that for some $\alpha>0$ the coercivity of the second variation of $J(x, u, \hat{\omega})$ at $(\hat{x}(\cdot), \hat{u}(\cdot))$ holds, that is,

$$
\hat{J}^{\prime \prime}(\eta, v) \geq \frac{\alpha}{2}\left\{|\eta(a)|^{2}+|\eta(b)|^{2}+\int_{a}^{b}\left(|\eta(t)|^{2}+|v(t)|^{2}\right) \mathrm{d} t\right\}
$$

for all $(\eta(\cdot), v(\cdot)) \in \mathrm{C}_{\mathrm{p}}^{1}\left([a, b], \mathbb{R}^{n}\right) \times \mathrm{C}_{\mathrm{p}}\left([a, b], \mathbb{R}^{m}\right)$ admissible for problem $\left(\mathrm{C}_{\hat{\omega}}\right)$, that is,

$$
\dot{\eta}(t)=\hat{\mathcal{A}}(t) \eta(t)+\hat{\mathcal{B}}(t) v(t), \quad \hat{N}(t) v(t)=0, \quad t \in[a, b], \quad \hat{M}\left(\begin{array}{l}
\eta(a) \\
\eta(b)
\end{array}\right)=0 .
$$

Then there exists $\varepsilon>0$ such that for all $\omega \in \mathbb{R}^{d}$ with $|\omega-\hat{\omega}|<\varepsilon$ the problem $\left(\mathrm{C}_{\omega}\right)$ has a strict weak local minimum $(x(\cdot, \omega), u(\cdot, \omega))$ with the associated multipliers $\lambda_{0}(\omega)=1, \gamma(\omega) \in \mathbb{R}^{r}, p(\cdot, \omega) \in \mathrm{C}_{\mathrm{p}}^{1}\left([a, b], \mathbb{R}^{n}\right), \lambda(\cdot, \omega) \in$ $\mathrm{C}_{\mathrm{p}}\left([a, b], \mathbb{R}^{k}\right)$ that are $\mathrm{C}^{1}$ functions in the argument $\omega$ and satisfy the weak Pontryagin principle corresponding to problem $\left(\mathrm{C}_{\omega}\right)$, that is,

$$
\begin{gathered}
-\dot{p}(t, \omega)=H_{x}^{T}(t, x(t, \omega), u(t, \omega), p(t, \omega), \lambda(t, \omega), \omega), \quad t \in[a, b], \\
H_{u}^{T}(t, x(t, \omega), u(t, \omega), p(t, \omega), \lambda(t, \omega), \omega)=0, \quad t \in[a, b],
\end{gathered}
$$




$$
\left(\begin{array}{c}
-p(a, \omega) \\
p(b, \omega)
\end{array}\right)=\nabla K^{T}(x(a, \omega), x(b, \omega), \omega)+\nabla \varphi^{T}(x(a, \omega), x(b, \omega), \omega) \gamma(\omega)
$$

Proof. The proof is based on applying the transformation introduced in Section 3 to the problem $\left(\mathrm{C}_{\omega}\right)$ in order to obtain an augmented problem $\left(\tilde{\mathrm{C}}_{\omega}\right)$ on the interval $[c, b]$ with separated state endpoints. With the help of the results developed in Section 3, we shall verify that all the hypotheses of Theorem 4.3 in [33] are satisfied by the transformed problem and hence, the sensitivity analysis result of that theorem is valid for problem $\left(\tilde{\mathrm{C}}_{\omega}\right)$. Then, we employ the inverse of this transformation to establish the statement of this theorem for our problem $\left(\mathrm{C}_{\omega}\right)$.

Since the feasible pair $(\hat{x}(\cdot), \hat{u}(\cdot))$ and its corresponding multipliers $\left(\lambda_{0}=1, \hat{\gamma}, \hat{p}(\cdot), \hat{\lambda}(\cdot)\right)$ satisfy the hypotheses of Theorem 3.3 in [33], it follows that $(\hat{x}(\cdot), \hat{u}(\cdot))$ is a strict weak local minimum for problem $\left(\mathrm{C}_{\hat{\omega}}\right)$.

Consider the following augmented nonlinear optimal control problem with separated endpoints, obtained by applying the transformation in Section 3 to our problem $\left(\mathrm{C}_{\omega}\right)$,

$$
\operatorname{minimize} \quad \tilde{J}(\tilde{x}, \tilde{u}, \omega):=K(\tilde{x}(b), \omega)+\int_{c}^{b} \tilde{L}(t, \tilde{x}(t), \tilde{u}(t), \omega) \mathrm{d} t
$$

subject to $\tilde{x}(\cdot) \in \mathrm{C}_{\mathrm{p}}^{1}\left([c, b], \mathbb{R}^{2 n}\right)$ and $\tilde{u}(\cdot) \in \mathrm{C}_{\mathrm{p}}^{1}\left([c, b], \mathbb{R}^{2 m}\right)$ partitioned as in (3.5) such that

$$
\begin{array}{cl}
\dot{\tilde{x}}(t)=\tilde{f}(t, \tilde{x}(t), \tilde{u}(t), \omega), \quad \tilde{\psi}(t, \tilde{u}(t), \omega)=0, \quad t \in[c, b], \\
\tilde{\varphi}_{c}(\tilde{x}(c), \omega):=\tilde{x}_{1}(c)-\tilde{x}_{2}(c)=0, \quad \tilde{\varphi}_{b}(\tilde{x}(b), \omega):=\varphi\left(\tilde{x}_{1}(b), \tilde{x}_{2}(b), \omega\right)=0 .
\end{array}
$$

The data $(\tilde{L}, \tilde{f}, \tilde{\psi})$ of problem $\left(\tilde{\mathrm{C}}_{\omega}\right)$ are defined in terms of the corresponding data $(L, f, \psi)$ of problem $\left(\mathrm{C}_{\omega}\right)$ via (3.4). Let $(\hat{\tilde{x}}(\cdot), \hat{\tilde{u}}(\cdot))$ be the pair obtained from $(\hat{x}(\cdot), \hat{u}(\cdot))$ through equations (3.10) and let its corresponding matrices $\hat{\tilde{M}}$ and $\hat{\tilde{N}}(t)$ for $t \in[c, b]$ be obtained from $\hat{M}$ and $\hat{N}(t)$ for $t \in[a, b]$ through (3.18). It is immediate that the pair $(\hat{\tilde{x}}(\cdot), \hat{\tilde{u}}(\cdot))$ together with $\hat{\omega}$ satisfies the assumption $(\hat{\tilde{A}} 2)$, that corresponds to $(\hat{\mathrm{A}} 2)$ by replacing the data of $\left(\mathrm{C}_{\omega}\right)$ with the data for $\left(\tilde{\mathrm{C}}_{\omega}\right)$. Furthermore, by Proposition 3.1 and Corollary $3.2,(\tilde{\tilde{\tilde{x}}}(\cdot), \hat{\tilde{u}}(\cdot))$ is a feasible pair, which is a strict weak local minimum for problem $\left(\tilde{\mathrm{C}}_{\hat{\omega}}\right)$. Then Proposition 3.4 yields that $(\hat{\tilde{x}}(\cdot), \hat{\tilde{u}}(\cdot))$ satisfies the weak Pontryagin principle with the multipliers $\left(\tilde{\lambda} \tilde{\lambda}_{0}=1, \hat{\tilde{\gamma}}, \hat{\tilde{p}}(\cdot), \hat{\tilde{\lambda}}(\cdot)\right)$ defined from the multipliers $\left(\lambda_{0}=1, \hat{\gamma}, \hat{p}(\cdot), \hat{\lambda}(\cdot)\right)$ through equation (3.12). The normality of problem $\left(\tilde{\mathrm{C}}_{\hat{\omega}}\right)$ at $(\hat{\tilde{x}}(\cdot), \hat{\tilde{u}}(\cdot))$ is a result of Corollary 3.5. Now, by Remark 2.9, we have that the coercivity of the functional $\hat{J}^{\prime \prime}(\eta, v)$ in $(4.1)$ implies that the corresponding strengthened Legendre-Clebsch condition at $(\hat{x}(\cdot), \hat{u}(\cdot))$ for problem $\left(\mathrm{C}_{\hat{\omega}}\right)$ holds, i.e., $\hat{Y}^{T}(t) \hat{R}(t) \hat{Y}(t)>0$ on $[a, b]$. Thus, using Propositions 3.10 and 3.8 , it results that the strengthened LegendreClebsch condition is satisfied at $\left(\hat{\tilde{x}}(\cdot), \hat{\tilde{u}}(\cdot), \hat{\tilde{p}}(\cdot), \hat{\tilde{\lambda}}(\cdot), \hat{\tilde{\gamma}}, \tilde{\lambda}_{0}=1\right)$ for problem $\left(\widetilde{\mathrm{C}}_{\hat{\omega}}\right)$ and the second variation $\hat{\tilde{J}}^{\prime \prime}(\tilde{\eta}, \tilde{v})$ of $\tilde{J}(\tilde{x}, \tilde{u}, \hat{\omega})$ at $(\hat{\tilde{x}}(\cdot), \hat{\tilde{u}}(\cdot))$ is coercive over all admissible pairs $(\tilde{\eta}(\cdot), \tilde{v}(\cdot)) \in \mathrm{C}_{\mathrm{p}}^{1}\left([c, b], \mathbb{R}^{2 n}\right) \times \mathrm{C}_{\mathrm{p}}\left([c, b], \mathbb{R}^{2 m}\right)$. Hence, by Remark 2.8, the coercivity of $\hat{\tilde{J}}_{\tilde{C}}^{\prime \prime}(\tilde{\eta}, \tilde{v})$ also holds over all pairs $(\tilde{\eta}(\cdot), \tilde{v}(\cdot)) \in W^{1,2}\left([c, b], \mathbb{R}^{2 n}\right) \times L^{2}\left([c, b], \mathbb{R}^{2 m}\right)$ that are admissible for problem $\left(\tilde{\mathrm{C}}_{\hat{\omega}}\right)$ at $(\hat{\tilde{x}}(\cdot), \hat{\tilde{u}}(\cdot))$. Consequently, all the assumptions of Theorem 4.3 in [33] are satisfied at $(\hat{\tilde{x}}(\cdot), \hat{\tilde{u}}(\cdot))$ for problem $\left(\tilde{\mathrm{C}}_{\hat{\omega}}\right)$ and hence, by the same theorem, there exists $\varepsilon>0$ such that for all $\omega \in \mathbb{R}^{d}$ with $|\omega-\hat{\omega}|<\varepsilon$ the problem $\left(\tilde{\mathrm{C}}_{\omega}\right)$ has a strict weak local minimum $(\tilde{x}(\cdot, \omega), \tilde{u}(\cdot, \omega))$ with the multipliers $\tilde{\lambda}_{0}(\omega)=1, \tilde{\gamma}(\omega) \in \mathbb{R}^{n+r}, \tilde{p}(\cdot, \omega) \in \mathrm{C}_{\mathrm{p}}^{1}\left([c, b], \mathbb{R}^{2 n}\right), \tilde{\lambda}(\cdot, \omega) \in \mathrm{C}_{\mathrm{p}}\left([c, b], \mathbb{R}^{2 k}\right)$, which are $\mathrm{C}^{1}$ functions in the argument $\omega$ and satisfy the corresponding weak Pontryagin principle.

Now, for each such $\omega$, let $(x(\cdot, \omega), u(\cdot, \omega))$ be the pair obtained from $(\tilde{x}(\cdot, \omega), \tilde{u}(\cdot, \omega))$ via $(3.11)$ and let $\lambda_{0}(\omega)=$ $1, \gamma(\omega) \in \mathbb{R}^{r}, p(\cdot, \omega) \in \mathrm{C}_{\mathrm{p}}^{1}\left([a, b], \mathbb{R}^{n}\right)$, and $\lambda(\cdot, \omega) \in \mathrm{C}_{\mathrm{p}}\left([a, b], \mathbb{R}^{k}\right)$ be the multipliers obtained from $\tilde{\lambda}_{0}(\omega)=1$, $\tilde{\gamma}(\omega), \tilde{p}(\cdot, \omega)$, and $\tilde{\lambda}(\cdot, \omega))$ via $(3.20)$. By the converse statements in Propositions 3.1 and 3.4 and in Corollaries 3.2 and 3.5 , the pair $(x(\cdot, \omega), u(\cdot, \omega))$ is a strict weak local minimum for problem $\left(\mathrm{C}_{\omega}\right)$, at which the problem is normal and together with the multipliers $\left(\lambda_{0}=1, \gamma(\omega), p(\cdot, \omega), \lambda(\cdot, \omega)\right)$ satisfies the weak Pontryagin principle for problem $\left(\mathrm{C}_{\omega}\right)$, that is, equations (4.2)-(4.4) are satisfied. The fact that $(x(t, \omega), u(t, \omega))$ and $(\gamma(\omega), p(t, \omega), \lambda(t, \omega))$ are $\mathrm{C}^{1}$ functions in the argument $\omega$ over the set $B_{\varepsilon}(\hat{\omega})$ for every $t \in[a, b]$ follows directly from this property 
being true for $(\tilde{x}(t, \omega), \tilde{u}(t, \omega))$ and $(\tilde{\gamma}(\omega), \tilde{p}(t, \omega), \tilde{\lambda}(t, \omega))$ and from formulas (3.11) and (3.20). The proof is complete.

\section{Optimal Control Problem With free time}

In this section we discuss the transformation from Section 3 for optimal control problems with free initial and final times. In particular, we show that this transformation preserves the corresponding strong or weak Pontryagin principle. To simplify the presentation, we focus on the autonomous case. However, the nonautonomous case is also handled with the techniques of this section and the difference between the results for the autonomous and nonautonomous settings is elaborated in Remarks 5.2, 5.3, 5.5, and 5.7. Thus, we consider the nonlinear optimal control problem

$$
\operatorname{minimize} \quad J(a, b, x, u):=K(a, b, x(a), x(b))+\int_{a}^{b} L(x(t), u(t)) \mathrm{d} t
$$

subject to $x(\cdot) \in \mathrm{C}_{\mathrm{p}}^{1}\left([a, b], \mathbb{R}^{n}\right)$ and $u(\cdot) \in \mathrm{C}_{\mathrm{p}}\left([a, b], \mathbb{R}^{m}\right)$ such that

$$
\dot{x}(t)=f(x(t), u(t)), \quad \psi(u(t))=0, \quad t \in[a, b], \quad \varphi(a, b, x(a), x(b))=0 .
$$

The functions $L, f, \psi$ have the same dimensions as in the problem $(\mathrm{C})$ from Section 2 with the distinction that they do not explicitly depend on the variable $t$. On the other hand, the functions $K$ and $\varphi$ depend on the varying initial and final times $a$ and $b$. The Hamiltonian $H$ is also defined via (2.4) with the data of problem (P).

We say that $(\hat{a}, \hat{b}, \hat{x}(\cdot), \hat{u}(\cdot))$ is a strong local minimum for problem $(\mathrm{P})$, if there exists $\delta>0$ such that $J(a, b, x, u) \geq J(\hat{a}, \hat{b}, \hat{x}, \hat{u})$ for all feasible $(a, b, x(\cdot), u(\cdot))$ for $(\mathrm{P})$ with $|a-\hat{a}|<\delta,|b-\hat{b}|<\delta$, and $\|x(\cdot)-\hat{x}(\cdot)\|_{\infty}<$ $\delta$. For the weak local minimum we replace the latter norm by $\|(x(\cdot)-\hat{x}(\cdot), u(\cdot)-\hat{u}(\cdot))\|_{\infty}<\delta$. As the pairs $(x(\cdot), u(\cdot))$ and $(\hat{x}(\cdot), \hat{u}(\cdot))$ are defined on different intervals, we need to define properly the above norms. This is done by extending $(x(\cdot), u(\cdot))$ outside the interval $[a, b]$ as

$$
(x(t), u(t)):= \begin{cases}\left(x(a), u\left(a^{+}\right)\right), & \text {for } t<a \\ \left(x(b), u\left(b^{-}\right)\right), & \text {for } t>b .\end{cases}
$$

Similarly we extend $(\hat{x}(\cdot), \hat{u}(\cdot))$ outside the interval $[\hat{a}, \hat{b}]$. This means that the norm $\|\cdot\|_{\infty}$ in the aforementioned local optimality definitions is taken over the extended time interval $[\min \{a, \hat{a}\}, \max \{b, \hat{b}\}]$. For a function $\hat{z}(\cdot) \in$ $\mathrm{C}_{\mathrm{p}}\left([\hat{a}, \hat{b}], \mathbb{R}^{d}\right)$ and $\varepsilon>0$ we define the set

$$
\mathcal{P} T_{\varepsilon}(\hat{z}):=\left\{z \in \mathbb{R}^{d} \text { such that } \exists t \in[\hat{a}-\varepsilon, \hat{b}+\varepsilon]:|z-\hat{z}(t)|<\varepsilon\right\}
$$

Parallel to (A0) and (A1), we make the following assumptions for the regularity of the data $h(x, u):=$ $(L(x, u), f(x, u), \psi(u)), K(a, b, x, y)$, and $\varphi(a, b, x, y)$ of problem (P) near a reference feasible quadruplet $(\hat{a}, \hat{b}, \hat{x}(\cdot), \hat{u}(\cdot))$.

(A0) There exists $\delta_{1}>0$ such that for each $u$ the function $h(\cdot, u)$ is differentiable on $\mathcal{P} T_{\delta_{1}}(\hat{x})$ and the functions $h$ and $\nabla_{x} h$ are continuous; the functions $K$ and $\varphi$ are continuously differentiable on $B_{\delta_{1}}(\hat{a}, \hat{b}, \hat{x}(a), \hat{x}(b))$ and the matrix $\nabla \varphi(\hat{a}, \hat{b}, \hat{x}(a), \hat{x}(b))$ has full rank.

(A1) There exists $\delta_{1}>0$ such that the function $h(\cdot, \cdot)$ is differentiable on $\mathcal{P} T_{\delta_{1}}(\hat{x}, \hat{u})$ and the functions $h$ and $\nabla h$ are continuous; the functions $K$ and $\varphi$ are continuously differentiable on $B_{\delta_{1}}(\hat{a}, \hat{b}, \hat{x}(a), \hat{x}(b))$ and the matrices $\nabla \varphi(\hat{a}, \hat{b}, \hat{x}(a), \hat{x}(b))$ and $\nabla \psi(\hat{u}(t))$ for $t \in[\hat{a}, \hat{b}]$ have full rank. 
The following strong Pontryagin principle for problem (P), compare with Definition 2.1, is derived in Theorem 5.1 of [11] or Theorem 8.7.2 in [37]. If $(\hat{a}, \hat{b}, \hat{x}(\cdot), \hat{u}(\cdot))$ is a strong local minimum for problem (P) at which $(\mathbb{A} 0)$ holds, then there exist a constant $\lambda_{0} \geq 0$, a vector $\gamma \in \mathbb{R}^{r}$, and a function $p(\cdot) \in \mathrm{C}_{\mathrm{p}}^{1}\left([\hat{a}, \hat{b}], \mathbb{R}^{n}\right)$ satisfying

$$
\begin{gathered}
\lambda_{0}+\|p(\cdot)\|_{\mathrm{C}_{\mathrm{p}}[\hat{a}, \hat{b}]} \neq 0, \\
-\dot{p}(t)=\mathcal{A}^{T}(t) p(t)+\lambda_{0} L_{x}^{T}(\hat{x}(t), \hat{u}(t)), \quad t \in[\hat{a}, \hat{b}], \\
\left(\begin{array}{c}
H\left(\hat{x}(\hat{a}), \hat{u}(\hat{a}), p(\hat{a}), 0, \lambda_{0}\right) \\
-H\left(\hat{x}(\hat{b}), \hat{u}(\hat{b}), p(\hat{b}), 0, \lambda_{0}\right) \\
-p(\hat{a}) \\
p(\hat{b})
\end{array}\right)=\lambda_{0} \nabla K^{T}(\hat{a}, \hat{b}, \hat{x}(\hat{a}), \hat{x}(\hat{b}))+M^{T} \gamma, \\
\min _{u \in \mathbb{R}^{m}, \psi(u)=0}^{H}\left(\hat{x}(t), u, p(t), 0, \lambda_{0}\right) \quad \text { is attained at } \hat{u}(t), t \in[\hat{a}, \hat{b}],
\end{gathered}
$$

and in addition the free initial/final time condition

$$
\begin{aligned}
H\left(\hat{x}(t), \hat{u}(t), p(t), 0, \lambda_{0}\right) & =\lambda_{0} K_{t_{1}}(\hat{a}, \hat{b}, \hat{x}(\hat{a}), \hat{x}(\hat{b}))+\varphi_{t_{1}}^{T}(\hat{a}, \hat{b}, \hat{x}(\hat{a}), \hat{x}(\hat{b})) \gamma, \quad t \in[\hat{a}, \hat{b}], \\
& =-\lambda_{0} K_{t_{2}}(\hat{a}, \hat{b}, \hat{x}(\hat{a}), \hat{x}(\hat{b}))-\varphi_{t_{2}}^{T}(\hat{a}, \hat{b}, \hat{x}(\hat{a}), \hat{x}(\hat{b})) \gamma, \quad t \in[\hat{a}, \hat{b}]
\end{aligned}
$$

Equations (5.5)-(5.6) are equivalent to saying that $H\left(\hat{x}(t), \hat{u}(t), p(t), 0, \lambda_{0}\right)$ is a constant function on $[\hat{a}, \hat{b}]$ and the first two equations in (5.3) hold. The matrices $\mathcal{A}(t)$ and $M$ appearing in (5.2) and (5.3) are defined similarly to (2.6), i.e., $\mathcal{A}(t)=f_{x}(\hat{x}(t), \hat{u}(t))$ on $[\hat{a}, \hat{b}]$ and $M=\nabla \varphi^{T}(\hat{a}, \hat{b}, \hat{x}(\hat{a}), \hat{x}(\hat{b}))$, where the gradient is taken with respect to all four variables. The subscripts $t_{1}$ in (5.5) and $t_{2}$ in (5.6) denote the partial derivatives of the given functions with respect to their first and second variables. If $(\hat{a}, \hat{b}, \hat{x}(\cdot), \hat{u}(\cdot))$ is instead a weak local minimum for problem $(\mathrm{P})$ with assumption $(\mathbb{A} 1)$ holding at $(\hat{a}, \hat{b}, \hat{x}(\cdot), \hat{u}(\cdot))$, then the local version of above minimality condition (5.4) implies that in addition there exists a function $\lambda(\cdot) \in \mathrm{C}_{\mathrm{p}}\left([a, b], \mathbb{R}^{k}\right)$ such that

$$
\mathcal{B}^{T}(t) p(t)+\lambda_{0} L_{u}^{T}(\hat{x}(t), \hat{u}(t))+N^{T}(t) \lambda(t)=0, \quad t \in[\hat{a}, \hat{b}]
$$

where the matrices $\mathcal{B}(t)$ and $N(t)$ are defined according to $(2.6)$, i.e., $\mathcal{B}(t)=f_{u}(\hat{x}(t), \hat{u}(t))$ and $N(t)=\nabla \psi(\hat{u}(t))$ on $[\hat{a}, \hat{b}]$.

Remark 5.1. (i) Since $\psi(\hat{u}(t))=0$ for all $t \in[\hat{a}, \hat{b}]$, then the Hamiltonian on the left-hand side of the free time conditions in (5.5)-(5.6), referring to the weak Pontryagin principle for problem (P), can be replaced by $H\left(\hat{x}(t), \hat{u}(t), p(t), \lambda(t), \lambda_{0}\right)$. It follows that $H\left(\hat{x}(\cdot), \hat{u}(\cdot), p(\cdot), \lambda(\cdot), \lambda_{0}\right)$, being constant, is a continuous function on $[\hat{a}, \hat{b}]$, even though the control $\hat{u}(\cdot)$ is only piecewise continuous on $[\hat{a}, \hat{b}]$.

(ii) Conditions (5.5)-(5.6) show that if both functions $K$ and $\varphi$ are independent of $t_{1}$ or independent of $t_{2}$, then in the strong and weak Pontryagin principle for problem $(\mathrm{P})$ we have

$$
H\left(\hat{x}(t), \hat{u}(t), p(t), 0, \lambda_{0}\right) \equiv 0, \quad t \in[\hat{a}, \hat{b}] .
$$

In particular, condition (5.8) is satisfied in the strong and weak Pontryagin principle for problem $(\mathrm{P})$ if the initial time is fixed and the final time is free, or if the initial time is free and the final time is fixed.

Remark 5.2. When the data $L, f$, and $H$ in problem (P) depend explicitly on $t$ and the control set $U:=\{u \in$ $\left.\mathbb{R}^{m}: \psi(u)=0\right\}$ remains constant, that is, we have $L(t, x, u), f(t, x, u)$, and $H\left(t, x, u, p, \lambda, \lambda_{0}\right)$, then conditions (5.1)-(5.4) and (5.7) continue to be valid. However, it is well-known (see e.g. [11], Thm. 5.1) that in this case 
the Hamiltonian is no longer constant and instead of (5.5) and (5.6) we now have, for all $t \in[\hat{a}, \hat{b}]$,

$$
\begin{aligned}
H\left(t, \hat{x}(t), \hat{u}(t), p(t), 0, \lambda_{0}\right) & =\left(\lambda_{0} K_{t_{1}}+\varphi_{t_{1}}^{T} \gamma\right)(\hat{a}, \hat{b}, \hat{x}(\hat{a}), \hat{x}(\hat{b}))+\int_{\hat{a}}^{t} \hat{H}_{t}(s) \mathrm{d} s \\
& =-\left(\lambda_{0} K_{t_{2}}+\varphi_{t_{2}}^{T} \gamma\right)(\hat{a}, \hat{b}, \hat{x}(\hat{a}), \hat{x}(\hat{b}))-\int_{t}^{\hat{b}} \hat{H}_{t}(s) \mathrm{d} s
\end{aligned}
$$

where $\hat{H}_{t}(s):=H_{t}\left(s, \hat{x}(s), \hat{u}(s), p(s), 0, \lambda_{0}\right)$ for $s \in[\hat{a}, \hat{b}]$.

Based on the transformation presented in Section 3 we consider the augmented optimal control problem with separated state endpoints and separated initial and final times

$$
\operatorname{minimize} \quad \tilde{J}(c, b, \tilde{\tau}, \tilde{x}, \tilde{u}):=\tilde{K}_{c}(c, \tilde{\tau}(c), \tilde{x}(c))+\tilde{K}_{b}(b, \tilde{\tau}(b), \tilde{x}(b))+\int_{c}^{b} \tilde{L}(\tilde{x}(t), \tilde{u}(t)) \mathrm{d} t
$$

on the interval $[c, b]$, where $c=(a+b) / 2$ is given by (1.1) and where the state variables $(\tilde{\tau}(\cdot), \tilde{x}(\cdot)) \in$ $\mathrm{C}_{\mathrm{p}}^{1}([c, b], \mathbb{R}) \times \mathrm{C}_{\mathrm{p}}^{1}\left([c, b], \mathbb{R}^{2 n}\right)$ and the control $\tilde{u}(\cdot) \in \mathrm{C}_{\mathrm{p}}\left([c, b], \mathbb{R}^{2 m}\right)$ are such that

$$
\begin{gathered}
\dot{\tilde{\tau}}(t)=-1, \quad \dot{\tilde{x}}(t)=\tilde{f}(\tilde{x}(t), \tilde{u}(t)), \quad \tilde{\psi}(\tilde{u}(t))=0, \quad t \in[c, b], \\
\tilde{\varphi}_{c}(c, \tilde{\tau}(c), \tilde{x}(c))=0, \quad \tilde{\varphi}_{b}(b, \tilde{\tau}(b), \tilde{x}(b))=0 .
\end{gathered}
$$

Given the data $(L, f, \psi, K, \varphi)$ of problem $(\mathrm{P})$ we define the corresponding data of problem $(\tilde{\mathrm{P}})$ according to (3.4) and (3.5), i.e., we set

$$
\left.\begin{array}{c}
\tilde{L}(\tilde{x}, \tilde{u}):=L\left(\tilde{x}_{1},-\tilde{u}_{1}\right)+L\left(\tilde{x}_{2}, \tilde{u}_{2}\right), \\
\tilde{K}_{c}(c, \tilde{\tau}, \tilde{x}):=0, \quad \tilde{K}_{b}(b, \tilde{T}, \tilde{y}):=K\left(\tilde{T}, b, \tilde{y}_{1}, \tilde{y}_{2}\right), \\
\tilde{f}(\tilde{x}, \tilde{u}):=\left(\begin{array}{c}
-f\left(\tilde{x}_{1},-\tilde{u}_{1}\right) \\
f\left(\tilde{x}_{2}, \tilde{u}_{2}\right)
\end{array}\right), \quad \tilde{\psi}(\tilde{u}):=\left(\begin{array}{c}
\psi\left(-\tilde{u}_{1}\right) \\
\psi\left(\tilde{u}_{2}\right)
\end{array}\right), \\
\tilde{\varphi}_{c}(c, \tilde{\tau}, \tilde{x}):=\left(\begin{array}{c}
\tilde{\tau}-c \\
\tilde{x}_{1}-\tilde{x}_{2}
\end{array}\right), \quad \tilde{\varphi}_{b}(b, \tilde{T}, \tilde{y}):=\varphi\left(\tilde{T}, b, \tilde{y}_{1}, \tilde{y}_{2}\right), \\
\tilde{K}(c, b, \tilde{\tau}, \tilde{x}, \tilde{T}, \tilde{y}):=K\left(\tilde{T}, b, \tilde{y}_{1}, \tilde{y}_{2}\right), \quad \tilde{\varphi}(c, b, \tilde{\tau}, \tilde{x}, \tilde{T}, \tilde{y}):=\left(\begin{array}{c}
\tilde{\varphi}_{c}(c, \tilde{\tau}, \tilde{x}) \\
\tilde{\varphi}_{b}(b, \tilde{T}, \tilde{y})
\end{array}\right) .
\end{array}\right\}
$$

Note that since $\dot{\tilde{\tau}}(t)=-1$ on $[c, b]$, then it follows from the definition of $\tilde{\varphi}_{c}(c, \tilde{\tau}, \tilde{x})$ in (5.13) that $\tilde{\tau}(t)=2 c-t$ for all $t \in[c, b]$. The pseudo-Hamiltonian for problem $(\tilde{\mathrm{P}})$ is defined by

$$
\tilde{\mathcal{H}}\left(\tilde{\tau}, \tilde{x}, \tilde{u}, \tilde{q}, \tilde{p}, \tilde{\lambda}, \tilde{\lambda}_{0}\right):=-\tilde{q}+\tilde{H}\left(\tilde{x}, \tilde{u}, \tilde{p}, \tilde{\lambda}, \tilde{\lambda}_{0}\right),
$$

where the Hamiltonian of problem $(\tilde{\mathrm{P}})$ is

$$
\tilde{H}\left(\tilde{x}, \tilde{u}, \tilde{p}, \tilde{\lambda}, \tilde{\lambda}_{0}\right):=\tilde{p}^{T} \tilde{f}(\tilde{x}, \tilde{u})+\tilde{\lambda}_{0} \tilde{L}(\tilde{x}, \tilde{u})+\tilde{\lambda}^{T} \tilde{\psi}(\tilde{u}) .
$$

Remark 5.3. The functions $\tilde{L}, \tilde{f}, \tilde{\psi}$, and $\tilde{H}$ in (5.13) and (5.15) are autonomous, as they do not depend explicitly on the variable $t \in[c, b]$ and hence, they are independent of the state variable $\tilde{\tau}$. When the data $L, f$, and $H$ (but not $\psi$ ) for the original problem (P) are $t$-dependent, and so are the corresponding data for problem $(\tilde{\mathrm{P}})$, this case is also addressed in this paper in a similar manner. However, as we shall see in Remarks 5.5 and 5.7 , the functions $\tilde{L}, \tilde{f}$, and $\tilde{H}$ now depend also on the new state variable $\tilde{\tau}$. Furthermore, the 
adjoint function $\tilde{q}$ corresponding to the state variable $\tilde{\tau}$, and the Hamiltonian $\tilde{H}$ evaluated at the corresponding optimal solution, are not necessarily constant.

Note that since the endpoints $a$ and $b$ in the original problem $(\mathrm{P})$ are free and $c=(a+b) / 2$, then both endpoints $c$ and $b$ in the transformed problem $(\tilde{\mathrm{P}})$ are free as well. The endpoints constraints in (5.12) are separable in the state variables $(\tilde{\tau}$ and $\tilde{x})$ and in the initial and final time variables $(c$ and $b)$. From (5.13) we obtain that

$$
\nabla \tilde{K}(c, b, \tilde{\tau}, \tilde{x}, \tilde{T}, \tilde{y})=\left(\begin{array}{c}
0 \\
K_{t_{2}}\left(\tilde{T}, b, \tilde{y}_{1}, \tilde{y}_{2}\right) \\
0 \\
0 \\
0 \\
K_{t_{1}}\left(\tilde{T}, b, \tilde{y}_{1}, \tilde{y}_{2}\right) \\
\nabla_{(x, y)} K^{T}\left(\tilde{T}, b, \tilde{y}_{1}, \tilde{y}_{2}\right)
\end{array}\right)^{T}
$$

and

$$
\nabla \tilde{\varphi}(c, b, \tilde{\tau}, \tilde{x}, \tilde{T}, \tilde{y})=\left(\begin{array}{ccc}
-1 & 0 & 0 \\
0 & 0 & \varphi_{t_{2}}^{T}\left(\tilde{T}, b, \tilde{y}_{1}, \tilde{y}_{2}\right) \\
1 & 0 & 0 \\
0 & I & 0 \\
0 & -I & 0 \\
0 & 0 & \varphi_{t_{1}}^{T}\left(\tilde{T}, b, \tilde{y}_{1}, \tilde{y}_{2}\right) \\
0 & 0 & \nabla_{(x, y)} \varphi\left(\tilde{T}, b, \tilde{y}_{1}, \tilde{y}_{2}\right)
\end{array}\right)^{T}
$$

Let $(a, b, x(\cdot), u(\cdot))$ be feasible for problem $(\mathrm{P})$. Then in accordance with (3.10), Proposition 3.1, and the constraints of $(\tilde{\mathrm{P}})$ we deduce that $(c, b, \tilde{\tau}(\cdot), \tilde{x}(\cdot), \tilde{u}(\cdot))$ with

$$
\tilde{\tau}(t):=2 c-t, \quad \tilde{x}(t):=\left(\begin{array}{c}
x(2 c-t) \\
x(t)
\end{array}\right), \quad \tilde{u}(t):=\left(\begin{array}{c}
-u(2 c-t) \\
u(t)
\end{array}\right), \quad t \in[c, b],
$$

is feasible for problem $(\tilde{\mathrm{P}})$ and $\tilde{J}(c, b, \tilde{\tau}, \tilde{x}, \tilde{u})=J(a, b, x, u)$. Conversely, if $(c, b, \tilde{\tau}(\cdot), \tilde{x}(\cdot), \tilde{u}(\cdot))$ is feasible for problem $(\tilde{\mathrm{P}})$, then upon defining

$$
\tau(t):= \begin{cases}\tilde{\tau}(2 c-t), & t \in[a, c] \\ 2 c-\tilde{\tau}(t), & t \in[c, b]\end{cases}
$$

it is easy to check that $\tau$ is continuous on $[a, b], \tau(c)=c$, and $\dot{\tau}(t) \equiv 1$ on $[a, b]$, and thus $\tau(t)=t$, for all $t \in[a, b]$. Hence, by (3.11), Proposition 3.1, and the constraints of problem ( $\tilde{\mathrm{P}})$ we deduce that $(a, b, x(\cdot), u(\cdot))$ with $a:=2 c-b$ and

$$
x(t):=\left\{\begin{array}{ll}
\tilde{x}_{1}(2 c-t), & t \in[a, c], \\
\tilde{x}_{2}(t), & t \in[c, b],
\end{array} \quad u(t):= \begin{cases}-\tilde{u}_{1}(2 c-t), & t \in[a, c], \\
\tilde{u}_{2}(t), & t \in[c, b]\end{cases}\right.
$$

is feasible for problem $(\mathrm{P})$ and $J(a, b, x, u)=\tilde{J}(c, b, \tilde{\tau}, \tilde{x}, \tilde{u})$. By applying conditions (5.1)-(5.7) to problem $(\tilde{\mathrm{P}})$ we deduce the corresponding strong and weak Pontryagin principle. Namely, if $(c, b, \tilde{\tau}(\cdot), \tilde{x}(\cdot), \tilde{u}(\cdot))$ is a strong local 
minimum for problem $(\tilde{\mathrm{P}})$, then there exist $\tilde{\lambda}_{0} \geq 0, \tilde{\alpha} \in \mathbb{R}^{n+r+1}, \tilde{q}(\cdot) \in \mathrm{C}_{\mathrm{p}}^{1}([c, b], \mathbb{R})$, and $\tilde{p}(\cdot) \in \mathrm{C}_{\mathrm{p}}^{1}\left([c, b], \mathbb{R}^{2 n}\right)$ satisfying

$$
\begin{gathered}
\tilde{\lambda}_{0}+\|\tilde{q}(\cdot)\|_{\mathrm{C}_{\mathrm{p}}[c, b]}+\|\tilde{p}(\cdot)\|_{\mathrm{C}_{\mathrm{p}}[c, b]} \neq 0, \\
(-\dot{\tilde{q}}(t), \quad-\dot{\tilde{p}}(t))=\left(0, \quad \tilde{\mathcal{A}}^{T}(t) \tilde{p}(t)+\tilde{\lambda}_{0} \tilde{L}_{\tilde{x}}^{T}(\tilde{x}(t), \tilde{u}(t))\right), \quad t \in[c, b], \\
\left(\begin{array}{c}
\tilde{\mathcal{H}}\left(\tilde{\tau}(c), \tilde{x}(c), \tilde{u}(c), \tilde{q}(c), \tilde{p}(c), 0, \tilde{\lambda}_{0}\right) \\
-\tilde{\mathcal{H}}\left(\tilde{\tau}(b), \tilde{x}(b), \tilde{u}(b), \tilde{q}(b), \tilde{p}(b), 0, \tilde{\lambda}_{0}\right) \\
-\tilde{q}(c) \\
-\tilde{p}(c) \\
\tilde{q}(b) \\
\tilde{p}(b) \\
\tilde{\operatorname{Hin}}\left(\tilde{\tau}(t), \tilde{x}(t), \tilde{u}, \tilde{q}(t), \tilde{p}(t), 0, \tilde{\lambda}_{0}\right) \quad \text { is attained at } \tilde{u}(t), t \in[c, b],
\end{array}=\tilde{\lambda}_{0} \nabla \tilde{K}^{T}(c, b, \tilde{\tau}(c), \tilde{x}(c), \tilde{\tau}(b), \tilde{x}(b))+\tilde{M}^{T} \tilde{\alpha},\right.
\end{gathered}
$$

and the free initial/final time condition

$$
\begin{aligned}
\tilde{\mathcal{H}}\left(\tilde{\tau}(t), \tilde{x}(t), \tilde{u}(t), \tilde{q}(t), \tilde{p}(t), 0, \tilde{\lambda}_{0}\right) & =\text { constant for all } t \in[c, b], \\
& =\left(\tilde{\lambda}_{0} \tilde{K}_{t_{1}}+\tilde{\varphi}_{t_{1}}^{T} \tilde{\alpha}\right)(c, b, \tilde{\tau}(c), \tilde{x}(c), \tilde{\tau}(b), \tilde{x}(b)), \\
& =-\left(\tilde{\lambda}_{0} \tilde{K}_{t_{2}}+\tilde{\varphi}_{t_{2}}^{T} \tilde{\alpha}\right)(c, b, \tilde{\tau}(c), \tilde{x}(c), \tilde{\tau}(b), \tilde{x}(b)) .
\end{aligned}
$$

On the other hand, if $(c, b, \tilde{x}(\cdot), \tilde{u}(\cdot))$ is a weak local minimum of problem $(\tilde{\mathrm{P}})$, then the local version of $(5.23)$ implies the existence of $\tilde{\lambda}(\cdot) \in \mathrm{C}_{\mathrm{p}}\left([c, b], \mathbb{R}^{2 k}\right)$ satisfying

$$
\tilde{\mathcal{B}}^{T}(t) \tilde{p}(t)+\tilde{\lambda}_{0} \tilde{L}_{\tilde{u}}^{T}(\tilde{x}(t), \tilde{u}(t))+\tilde{N}^{T}(t) \tilde{\lambda}(t)=0, \quad t \in[c, b] .
$$

The matrices $\tilde{\mathcal{A}}(t), \tilde{\mathcal{B}}(t), \tilde{N}(t)$ in (5.21) and (5.27) are defined according to formulas (3.18) and (3.19) with the argument $2 c-t$ in the left upper block, and the matrix $\tilde{M}$ in $(5.22)$ is equal to $\nabla \tilde{\varphi}(c, b, \tilde{\tau}(c), \tilde{x}(c), \tilde{\tau}(b), \tilde{x}(b))$ in which the gradient is taken with respect to all six components.

As we shall see in the following lemma, the Pontryagin principle manifested by equations (5.20)-(5.26) can be reformulated merely in terms of $\tilde{\lambda}_{0}, \tilde{p}$, the Hamiltonian $\tilde{H}$, and the multipliers in $\tilde{\alpha}$ associated to the state $\tilde{x}(\cdot)$ in the endpoint constraints. That is, the multiplier in $\tilde{\alpha}$ and the constant adjoint variable $\tilde{q}$ corresponding to the state $\tilde{\tau}(\cdot)$ are superfluous.

Lemma 5.4 (Simplified version of strong Pontryagin principle for $(\tilde{\mathrm{P}}))$. If $(c, b, \tilde{\tau}(\cdot), \tilde{x}(\cdot), \tilde{u}(\cdot))$ is a strong local minimum for problem $(\tilde{\mathrm{P}})$, then the formulation of the strong Pontryagin principle in $(5.20)-(5.26)$ is equivalent to the existence of $\tilde{\lambda}_{0} \geq 0, \tilde{\gamma}:=\left(\tilde{\gamma}_{1}^{T}, \tilde{\gamma}_{2}^{T}\right)^{T} \in \mathbb{R}^{n+r}$, and $\tilde{p}(\cdot) \in \mathrm{C}_{\mathrm{p}}^{1}\left([c, b], \mathbb{R}^{2 n}\right)$ satisfying

$$
\begin{gathered}
\tilde{\lambda}_{0}+\|\tilde{p}(\cdot)\|_{\mathrm{C}_{\mathrm{p}}[c, b]} \neq 0, \\
-\dot{\tilde{p}}(t)=\tilde{\mathcal{A}}^{T}(t) \tilde{p}(t)+\tilde{\lambda}_{0} \tilde{L}_{\tilde{x}}^{T}(\tilde{x}(t), \tilde{u}(t)), \quad t \in[c, b], \\
\tilde{p}(c)=\left(-\tilde{\gamma}_{1}^{T}, \tilde{\gamma}_{1}^{T}\right)^{T}, \\
\left(\begin{array}{c}
\frac{1}{2} \tilde{H}\left(\tilde{x}(c), \tilde{u}(c), \tilde{p}(c), 0, \tilde{\lambda}_{0}\right) \\
-\frac{1}{2} \tilde{H}\left(\tilde{x}(b), \tilde{u}(b), \tilde{p}(b), 0, \tilde{\lambda}_{0}\right) \\
\tilde{p}(b)
\end{array}\right)=\nabla\left(\tilde{\lambda}_{0} K+\varphi^{T} \tilde{\gamma}_{2}\right)^{T}\left(\tilde{\tau}(b), b, \tilde{x}_{1}(b), \tilde{x}_{2}(b)\right), \\
\min _{\tilde{u} \in \mathbb{R}^{2 m}, \tilde{\psi}(\tilde{u})=0} \tilde{H}\left(\tilde{x}(t), \tilde{u}, \tilde{p}(t), 0, \tilde{\lambda}_{0}\right) \quad \text { is attained at } \tilde{u}(t), t \in[c, b],
\end{gathered}
$$


and

$$
\begin{aligned}
\tilde{H}\left(\tilde{x}(t), \tilde{u}(t), \tilde{p}(t), 0, \tilde{\lambda}_{0}\right) & =2\left(\tilde{\lambda}_{0} K_{t_{1}}+\varphi_{t_{1}}^{T} \tilde{\gamma}_{2}\right)\left(\tilde{\tau}(b), b, \tilde{x}_{1}(b), \tilde{x}_{2}(b)\right) \quad t \in[c, b], \\
& =-2\left(\tilde{\lambda}_{0} K_{t_{2}}+\varphi_{t_{2}}^{T} \tilde{\gamma}_{2}\right)\left(\tilde{\tau}(b), b, \tilde{x}_{1}(b), \tilde{x}_{2}(b)\right) \quad t \in[c, b]
\end{aligned}
$$

Proof. Assume that (5.20)-(5.26) are satisfied. From $\tilde{M}:=\nabla \tilde{\varphi}(c, b, \tilde{\tau}(c), \tilde{x}(c), \tilde{\tau}(b), \tilde{x}(b)),(5.14)-(5.17)$, and from $\tilde{\tau}(t)=2 c-t$ we obtain when splitting $\tilde{p}(t)=\left(\tilde{p}_{1}^{T}(t), \tilde{p}_{2}^{T}(t)\right)^{T}$ that $(5.22)$ is equivalent to

$$
\left(\begin{array}{c}
\tilde{H}\left(\tilde{x}(c), \tilde{u}(c), \tilde{p}(c), 0, \tilde{\lambda}_{0}\right) \\
-\tilde{H}\left(\tilde{x}(b), \tilde{u}(b), \tilde{p}(b), 0, \tilde{\lambda}_{0}\right) \\
-\tilde{q}(c) \\
-\tilde{p}_{1}(c) \\
-\tilde{p}_{2}(c) \\
\tilde{q}(b) \\
\tilde{p}(b)
\end{array}\right)=\left(\begin{array}{c}
\tilde{q}(c)-\tilde{\alpha}_{0} \\
-\tilde{q}(b)+\left(\tilde{\lambda}_{0} K_{t_{2}}+\varphi_{t_{2}}^{T} \tilde{\alpha}_{2}\right)\left(\tilde{\tau}(b), b, \tilde{x}_{1}(b), \tilde{x}_{2}(b)\right) \\
\tilde{\alpha}_{0} \\
\tilde{\alpha}_{1} \\
-\tilde{\alpha}_{1} \\
\left(\tilde{\lambda}_{0} K_{t_{1}}+\varphi_{t_{1}}^{T} \tilde{\alpha}_{2}\right)\left(\tilde{\tau}(b), b, \tilde{x}_{1}(b), \tilde{x}_{2}(b)\right) \\
\nabla_{(x, y)}\left(\tilde{\lambda}_{0} K+\varphi^{T} \tilde{\alpha}_{2}\right)^{T}\left(\tilde{\tau}(b), b, \tilde{x}_{1}(b), \tilde{x}_{2}(b)\right)
\end{array}\right),
$$

and (5.24)-(5.26) are equivalent to

$$
\begin{aligned}
\tilde{H}\left(\tilde{x}(t), \tilde{u}(t), \tilde{p}(t), 0, \tilde{\lambda}_{0}\right) & =\tilde{q}-\tilde{\alpha}_{0}, \quad t \in[c, b], \\
& =\tilde{q}-\left(\tilde{\lambda}_{0} K_{t_{2}}+\varphi_{t_{2}}^{T} \tilde{\alpha}_{2}\right)\left(\tilde{\tau}(b), b, \tilde{x}_{1}(b), \tilde{x}_{2}(b)\right), \quad t \in[c, b] .
\end{aligned}
$$

The first equation of (5.21) yields that $\tilde{q}(t) \equiv \tilde{q}$ is constant on $[c, b]$. From (5.15) and from the first and third equations in (5.35) it follows that when $\tilde{\lambda}_{0}=0$ and $\tilde{p}(\cdot) \equiv 0$, then $\tilde{q}=0$. Thus, the nondegeneracy condition (5.20) is equivalent to (5.28). The second component of the adjoint equation (5.21) is obviously (5.29).

Set $\left(\tilde{\gamma}_{1}, \tilde{\gamma}_{2}\right):=\left(\tilde{\alpha}_{1}, \tilde{\alpha}_{2}\right)$. The fourth, fifth, and seventh equations in (5.35) imply that $\tilde{p}(c)$ and $\tilde{p}(b)$ satisfy, respectively, (5.30) and the third equation in (5.31). Since $\tilde{q}$ is constant, the minimality in (5.23) is equivalent to (5.32). Furthermore, from (5.36), (5.37), and from the first three equations and the sixth equation of (5.35) we obtain that, for all $t \in[c, b]$,

$$
\tilde{H}\left(\tilde{x}(t), \tilde{u}(t), \tilde{p}(t), 0, \tilde{\lambda}_{0}\right)=\tilde{H}\left(\tilde{x}(c), \tilde{u}(c), \tilde{p}(c), 0, \tilde{\lambda}_{0}\right)=\tilde{H}\left(\tilde{x}(b), \tilde{u}(b), \tilde{p}(b), 0, \tilde{\lambda}_{0}\right)
$$

and

$$
\begin{aligned}
\tilde{H}\left(\tilde{x}(c), \tilde{u}(c), \tilde{p}(c), 0, \tilde{\lambda}_{0}\right) & =2 \tilde{\lambda}_{0} K_{t_{1}}+2 \varphi_{t_{1}}^{T} \tilde{\alpha}_{2}=-2 \tilde{\alpha}_{0}=2 \tilde{q}, \\
-\tilde{H}\left(\tilde{x}(b), \tilde{u}(b), \tilde{p}(b), 0, \tilde{\lambda}_{0}\right) & =\tilde{\lambda}_{0}\left(-K_{t_{1}}+K_{t_{2}}\right)+\left(-\varphi_{t_{1}}^{T}+\varphi_{t_{2}}^{T}\right) \tilde{\alpha}_{2},
\end{aligned}
$$

where the partial derivatives are evaluated at $\left(\tilde{\tau}(b), b, \tilde{x}_{1}(b), \tilde{x}_{2}(b)\right)$. It immediately results that

$$
\tilde{\lambda}_{0} K_{t_{1}}+\varphi_{t_{1}}^{T} \tilde{\alpha}_{2}=-\left(\tilde{\lambda}_{0} K_{t_{2}}+\varphi_{t_{2}}^{T} \tilde{\alpha}_{2}\right)
$$

with the partial derivatives evaluated again at $\left(\tilde{\tau}(b), b, \tilde{x}_{1}(b), \tilde{x}_{2}(b)\right)$. Hence, since $\tilde{\gamma}_{2}=\tilde{\alpha}_{2}$, the first two equations of (5.31) are also valid. In addition, it follows from (5.38)-(5.41) that (5.33) and (5.34) hold true.

Conversely, if $\tilde{\lambda}_{0}, \tilde{\gamma}=\left(\tilde{\gamma}_{1}^{T}, \tilde{\gamma}_{2}^{T}\right)^{T}$, and $\tilde{p}(\cdot)$ satisfy $(5.28)-(5.34)$, it is straightforward to show that $\tilde{\lambda}_{0}, \tilde{p}(\cdot)$, $\left(\tilde{\alpha}_{1}, \tilde{\alpha}_{2}\right):=\left(\tilde{\gamma}_{1}, \tilde{\gamma}_{2}\right)$, and $\tilde{q}=-\tilde{\alpha}_{0}:=\left(\tilde{\lambda}_{0} K_{t_{1}}+\varphi_{t_{1}}^{T} \tilde{\gamma}_{2}\right)\left(\tilde{\tau}(b), b, \tilde{x}_{1}(b), \tilde{x}_{2}(b)\right)$ satisfy (5.20)-(5.26). The proof is complete. 
Remark 5.5. Consider the case when the functions $L, f$, and $H$ depend explicitly on $t$, for which they enjoy the same regularity assumptions as for the variable $x$. Then

$$
\tilde{L}(t, \tilde{\tau}, \tilde{x}, \tilde{u}):=L\left(\tilde{\tau}, \tilde{x}_{1},-\tilde{u}_{1}\right)+L\left(t, \tilde{x}_{2}, \tilde{u}_{2}\right), \quad \tilde{f}(t, \tilde{\tau}, \tilde{x}, \tilde{u}):=\left(\begin{array}{c}
-f\left(\tilde{\tau}, \tilde{x}_{1},-\tilde{u}_{1}\right) \\
f\left(t, \tilde{x}_{2}, \tilde{u}_{2}\right)
\end{array}\right)
$$

are functions of $(t, \tilde{\tau}, \tilde{x}, \tilde{u})$, and $\tilde{\mathcal{H}}$ is a function of $\left(t, \tilde{\tau}, \tilde{x}, \tilde{u}, \tilde{q}, \tilde{p}, \lambda, \lambda_{0}\right)$, and $\tilde{H}$ is a function of $\left(t, \tilde{\tau}, \tilde{x}, \tilde{u}, \tilde{p}, \lambda, \lambda_{0}\right)$. Consequently, the adjoint equation $\dot{\tilde{q}} \equiv 0$ and equations (5.24)-(5.26) are no longer valid. Hence, the first equation of (5.21) and, parallel to (5.9)-(5.10), the free initial/final time condition (5.24)-(5.26) are now replaced by the following equations

$$
\begin{aligned}
& -\dot{\tilde{q}}(t)=\tilde{\tilde{\mathcal{H}}}_{\tilde{\tau}}(t)=\tilde{f}_{\tilde{\tau}}^{T}(t, \tilde{\tau}(t), \tilde{x}(t), \tilde{u}(t)) \tilde{p}(t)+\tilde{\lambda}_{0} \tilde{L}_{\tilde{\tau}}^{T}(t, \tilde{\tau}(t), \tilde{x}(t), \tilde{u}(t)), \quad t \in[c, b], \\
& \tilde{\tilde{\mathcal{H}}}(t)=\left(\tilde{\lambda}_{0} \tilde{K}_{t_{1}}+\tilde{\varphi}_{t_{1}}^{T} \tilde{\alpha}\right)(c, b, \tilde{\tau}(c), \tilde{x}(c), \tilde{\tau}(b), \tilde{x}(b))+\int_{c}^{t} \tilde{\tilde{\mathcal{H}}}_{t}(s) \mathrm{d} s, \quad t \in[c, b], \\
& =-\left(\tilde{\lambda}_{0} \tilde{K}_{t_{2}}+\tilde{\varphi}_{t_{2}}^{T} \tilde{\alpha}\right)(c, b, \tilde{\tau}(c), \tilde{x}(c), \tilde{\tau}(b), \tilde{x}(b))-\int_{t}^{b} \tilde{\mathcal{H}}_{t}(s) \mathrm{d} s, \quad t \in[c, b],
\end{aligned}
$$

where $\tilde{\tilde{\mathcal{H}}}_{\tilde{\tau}}(t), \tilde{\tilde{\mathcal{H}}}(t)$, and $\tilde{\tilde{\mathcal{H}}}_{t}(t)$ denote the evaluation at $\left(t, \tilde{\tau}(t), \tilde{x}(t), \tilde{u}(t), \tilde{q}(t), \tilde{p}(t), 0, \tilde{\lambda}_{0}\right)$ of the functions $\tilde{\mathcal{H}}_{\tilde{\tau}}$, $\tilde{\mathcal{H}}$, and $\tilde{\mathcal{H}}_{t}$. Thus, neither of the functions $\tilde{q}$ and $\tilde{\mathcal{H}}$ is constant. Nevertheless, calculations along the proof of Lemma 5.4 yield that the strong Pontryagin principle for the nonautonomous case given by the equations (5.20), (5.42), (5.21)(ii), (5.22), (5.23), and (5.43)-(5.44) can be simplified as in Lemma 5.4, with the difference that the first two equations in (5.31) and equations (5.33)-(5.34) are now replaced by

$$
\left(\begin{array}{c}
\frac{1}{2} \tilde{\tilde{H}}(c) \\
-\frac{1}{2} \tilde{\tilde{H}}(b)
\end{array}\right)=\left(\begin{array}{c}
\left(\tilde{\lambda}_{0} K_{t_{1}}+\varphi_{t_{1}}^{T} \tilde{\gamma}_{2}\right)(b)+\int_{c}^{b} \tilde{\tilde{H}}_{\tilde{\tau}}(s) \mathrm{d} s \\
\left(\tilde{\lambda}_{0} K_{t_{2}}+\varphi_{t_{2}}^{T} \tilde{\gamma}_{2}\right)(b)+\frac{1}{2} \int_{c}^{b}\left(\tilde{\tilde{H}}_{t}(s)+\tilde{\tilde{H}}_{\tilde{\tau}}(s)\right) \mathrm{d} s
\end{array}\right)
$$

and

$$
\begin{aligned}
\tilde{\tilde{H}}(t) & =2\left(\tilde{\lambda}_{0} K_{t_{1}}+\varphi_{t_{1}}^{T} \tilde{\gamma}_{2}\right)(b)+2 \int_{c}^{b} \tilde{\tilde{H}}_{\tilde{\tau}}(s) \mathrm{d} s+\int_{c}^{t}\left(\tilde{\tilde{H}}_{t}(s)-\tilde{\tilde{H}}_{\tilde{\tau}}(s)\right) \mathrm{d} s \\
& =-2\left(\tilde{\lambda}_{0} K_{t_{2}}+\varphi_{t_{2}}^{T} \tilde{\gamma}_{2}\right)(b)-\int_{c}^{b}\left(\tilde{\tilde{H}}_{t}(s)+\tilde{\tilde{H}}_{\tilde{\tau}}(s)\right) \mathrm{d} s-\int_{t}^{b}\left(\tilde{\tilde{H}}_{t}(s)-\tilde{\tilde{H}}_{\tilde{\tau}}(s)\right) \mathrm{d} s,
\end{aligned}
$$

where $\tilde{\tilde{H}}(t), \tilde{\tilde{H}}_{\tilde{\tau}}(t)$, and $\tilde{\tilde{H}}_{t}(t)$ denote the functions $\tilde{H}, \tilde{H}_{\tilde{\tau}}$, and $\tilde{H}_{t}$ being evaluated at the quantity $\left(t, \tilde{\tau}(t), \tilde{x}(t), \tilde{u}(t), \tilde{p}(t), 0, \tilde{\lambda}_{0}\right)$, and where $\left(\tilde{\lambda}_{0} K_{t_{1}}+\varphi_{t_{1}}^{T} \tilde{\gamma}_{2}\right)(b)$ and $\left(\tilde{\lambda}_{0} K_{t_{2}}+\varphi_{t_{2}}^{T} \tilde{\gamma}_{2}\right)(b)$ stand for the evaluation of these functions at $\left(\tilde{\tau}(b), b, \tilde{x}_{1}(b), \tilde{x}_{2}(b)\right)$. When $L, f$, and $H$ are independent of $t$, then the functions $\tilde{L}$, $\tilde{f}$, and $\tilde{H}$ are independent of $t$ and $\tilde{\tau}$ and hence, equations (5.45)-(5.47) reduce to the corresponding ones in Lemma 5.4.

In the following result we show that the transformation from Section 3 is suitable also for problems with free initial and/or final time.

Proposition 5.6 (Strong Pontryagin principle). Assume that $(a, b, x(\cdot), u(\cdot))$ satisfies the strong Pontryagin principle for problem $(\mathrm{P})$, i.e., conditions (5.1)-(5.6) hold with multipliers $\lambda_{0}, \gamma, p(\cdot)$. Then for $c:=(a+$ $b) / 2$ and for the triplet $(\tilde{\tau}(\cdot), \tilde{x}(\cdot), \tilde{u}(\cdot))$ defined by $(5.18)$ we have that $(c, b, \tilde{\tau}(\cdot), \tilde{x}(\cdot), \tilde{u}(\cdot))$ satisfies the strong Pontryagin principle for problem $(\tilde{\mathrm{P}})$, i.e., conditions (5.28)-(5.34) are satisfied with the multipliers $\tilde{\lambda}_{0}, \tilde{\gamma}, \tilde{p}(\cdot)$ 
defined by (3.12), i.e.,

$$
\tilde{\lambda}_{0}:=\lambda_{0}, \quad \tilde{\gamma}:=\left(\begin{array}{c}
p(c) \\
\gamma
\end{array}\right), \quad \tilde{p}(t):=\left(\begin{array}{c}
-p(2 c-t) \\
p(t)
\end{array}\right), \quad t \in[c, b] .
$$

Conversely, assume that $(c, b, \tilde{\tau}(\cdot), \tilde{x}(\cdot), \tilde{u}(\cdot))$ satisfies the strong Pontryagin principle for problem $(\tilde{\mathrm{P}})$, i.e., conditions (5.28)-(5.34) are satisfied with multipliers $\tilde{\lambda}_{0}, \tilde{\gamma}, \tilde{p}(\cdot)$. Then for $a:=2 c-b$ and for the pair $(x(\cdot), u(\cdot))$ defined by (5.19) we have that $(a, b, x(\cdot), u(\cdot))$ satisfies the strong Pontryagin principle for problem $(\mathrm{P})$, i.e., conditions (5.1)-(5.5) are satisfied with the multipliers $\lambda_{0}, \gamma, p(\cdot)$ defined by $(3.20)$, i.e.,

$$
\lambda_{0}:=\tilde{\lambda}_{0}, \quad \gamma:=\tilde{\gamma}_{2}, \quad p(t):= \begin{cases}-\tilde{p}_{1}(2 c-t), & t \in[a, c], \\ \tilde{p}_{2}(t), & t \in[c, b] .\end{cases}
$$

Proof. Given a feasible $(a, b, x(\cdot), u(\cdot))$ for problem (P) satisfying conditions (5.1)-(5.6), then the triplet $(\tilde{\tau}(\cdot), \tilde{x}(\cdot), \tilde{u}(\cdot))$ defined by $(5.18)$ is feasible for problem $(\tilde{\mathrm{P}})$ and with the multipliers $\tilde{\lambda}_{0}, \tilde{\gamma}, \tilde{p}(\cdot)$ defined by (5.48) it is easy seen that it satisfies conditions (5.28)-(5.32) and the third equation in (5.31), see the proof of Proposition 3.3. In addition, condition (5.5) implies through (3.8) that for $t \in[c, b]$

$$
\begin{aligned}
\tilde{H}\left(\tilde{x}(t), \tilde{u}(t), \tilde{p}(t), 0, \tilde{\lambda}_{0}\right) & \\
& \stackrel{(3.8)}{=} H\left(\tilde{x}_{1}(t),-\tilde{u}_{1}(t),-\tilde{p}_{1}(t), 0, \tilde{\lambda}_{0}\right)+H\left(\tilde{x}_{2}(t), \tilde{u}_{2}(t), \tilde{p}_{2}(t), 0, \tilde{\lambda}_{0}\right) \\
& \stackrel{(5.18),(5.48)}{=} H\left(x(2 c-t), u(2 c-t), p(2 c-t), 0, \lambda_{0}\right)+H\left(x(t), u(t), p(t), 0, \lambda_{0}\right) \\
& \stackrel{(5.5)}{=} 2 \lambda_{0} K_{t_{1}}(a, b, x(a), x(b))+2 \varphi_{t_{1}}^{T}(a, b, x(a), x(b)) \gamma \\
& \stackrel{(5.18),(5.48)}{=} 2 \tilde{\lambda}_{0} K_{t_{1}}\left(\tilde{\tau}(b), b, \tilde{x}_{1}(b), \tilde{x}_{2}(b)\right)+2 \varphi_{t_{1}}^{T}\left(\tilde{\tau}(b), b, \tilde{x}_{1}(b), \tilde{x}_{2}(b)\right) \tilde{\gamma}_{2} .
\end{aligned}
$$

This shows the validity of condition (5.33). Using in the above calculation (5.6) instead of (5.5), we also obtain the validity of (5.34), completing the proof of the first part.

Conversely, assume that a feasible $(c, b, \tilde{\tau}(\cdot), \tilde{x}(\cdot), \tilde{u}(\cdot))$ for problem $(\tilde{\mathrm{P}})$ satisfies $(5.28)-(5.34)$ with the corresponding multipliers $\tilde{\lambda}_{0}, \tilde{\gamma}, \tilde{p}(\cdot)$. Then the pair $(x(\cdot), u(\cdot))$ defined by $(5.19)$ is feasible for problem $(\mathrm{P})$. We define the multipliers $\lambda_{0}, \gamma, p(\cdot)$ via (5.49). Then (5.30) yields that the function $p(\cdot)$ is continuous at $t=c$, and hence $p(\cdot) \in \mathrm{C}_{\mathrm{p}}^{1}\left([a, b], \mathbb{R}^{n}\right)$. Moreover, as in the proof of Proposition 3.3 we deduce the validity of conditions (5.1), (5.2), (5.4), and

$$
\begin{gathered}
\left(\begin{array}{c}
-p(a) \\
p(b)
\end{array}\right) \stackrel{(5.49)}{=} \tilde{p}(b) \stackrel{(5.31)}{=} \tilde{\lambda}_{0} \nabla_{(x, y)} K^{T}\left(\tilde{\tau}(b), b, \tilde{x}_{1}(b), \tilde{x}_{2}(b)\right)+\nabla_{(x, y)} \varphi^{T}\left(\tilde{\tau}(b), b, \tilde{x}_{1}(b), \tilde{x}_{2}(b)\right) \tilde{\gamma}_{2} \\
\stackrel{(5.19),(5.49)}{=} \lambda_{0} \nabla_{(x, y)} K^{T}(a, b, x(a), x(b))+\nabla_{(x, y)} \varphi^{T}(a, b, x(a), x(b)) \gamma,
\end{gathered}
$$

proving that the third and fourth equations in (5.3) are valid. On the other hand, the definition of the Hamiltonian $\tilde{H}$ in (5.15) with (5.13) yields that

$$
\tilde{H}\left(\tilde{x}(c), \tilde{u}(c), \tilde{p}(c), 0, \tilde{\lambda}_{0}\right)=2 H\left(x(c), u(c), p(c), 0, \lambda_{0}\right) .
$$

Set $h(t, u):=H\left(x(t), u, p(t), 0, \lambda_{0}\right)$ for $t \in[a, b]$ and $u \in \mathbb{R}^{m}$. Then the function $h(\cdot, \cdot)$ is continuous and having continuous $t$-partial derivative

$$
h_{t}(t, u)=H_{x}\left(x(t), u, p(t), 0, \lambda_{0}\right) \dot{x}(t)+H_{p}\left(x(t), u, p(t), 0, \lambda_{0}\right) \dot{p}(t) .
$$


Consider the closed set $U:=\left\{u \in \mathbb{R}^{m}, \psi(u)=0\right\}$. We redefine the function $u(\cdot) \in \mathrm{C}_{\mathrm{p}}\left([a, b], \mathbb{R}^{m}\right)$ so that it is also left-continuous on $[a, b]$. From the already proven minimality condition (5.4) we have that

$$
\min _{u \in U} h(t, u)=h(t, u(t)) \quad \text { for all } t \in[a, b]
$$

By Lemma 15.1 of $[11]$ we obtain that the function $h(\cdot, u(\cdot)) \in \mathrm{C}_{\mathrm{p}}^{1}([a, b], \mathbb{R})$ and

$$
h(t, u(t))=h(c, u(c))+\int_{c}^{t} h_{t}(\tau, u(\tau)) \mathrm{d} \tau, \quad t \in[a, b] .
$$

But from (5.54) and (2.12) we deduce that

$$
\begin{aligned}
h_{t}(\tau, u(\tau)) & =H_{x}\left(x(\tau), u(\tau), p(\tau), 0, \lambda_{0}\right) \dot{x}(\tau)+H_{p}\left(x(\tau), u(\tau), p(\tau), 0, \lambda_{0}\right) \dot{p}(\tau) \\
& =-\dot{p}^{T}(\tau) f(x(\tau), u(\tau))+[f(x(\tau), u(\tau))]^{T} \dot{p}(\tau)=0, \quad \tau \in[a, b] .
\end{aligned}
$$

Therefore, we derive that for all $t \in[a, b]$ we have

$$
\begin{aligned}
H\left(x(t), u(t), p(t), 0, \lambda_{0}\right) & =h(t, u(t)) \stackrel{(5.55)}{=} h(c, u(c))=H\left(x(c), u(c), p(c), 0, \lambda_{0}\right) \\
& \stackrel{(5.53)}{=} \frac{1}{2} \tilde{H}\left(\tilde{x}(c), \tilde{u}(c), \tilde{p}(c), 0, \tilde{\lambda}_{0}\right) \\
\stackrel{(5.33)}{=} & \tilde{\lambda}_{0} K_{t_{1}}\left(\tilde{\tau}(b), b, \tilde{x}_{1}(b), \tilde{x}_{2}(b)\right)+\varphi_{t_{1}}^{T}\left(\tilde{\tau}(b), b, \tilde{x}_{1}(b), \tilde{x}_{2}(b)\right) \tilde{\gamma}_{2} \\
\stackrel{(5.19),(5.49)}{=} & \lambda_{0} K_{t_{1}}(a, b, x(a), x(b))+\varphi_{t_{1}}^{T}(a, b, x(a), x(b)) \gamma,
\end{aligned}
$$

showing that condition (5.5) holds. Using the same calculation just performed and using (5.34) instead of (5.33), we reach to (5.6). Having (5.5) and (5.6) valid, we obtain that the first two equations of (5.3) hold true. The proof is now complete.

Remark 5.7. For the case where the functions $L$ and $f$ are explicitly depending on $t$, the statements in Proposition 5.6 remain valid when replacing equations (5.5)-(5.6) by (5.9)-(5.10), and equations (5.31) and (5.33)-(5.34) by (5.45)-(5.47). This requires some modifications in the proof of Proposition 5.6 based on the following easily derived identities

$$
\int_{c}^{\beta} \tilde{\tilde{H}}_{t}(s) \mathrm{d} s=\int_{c}^{\beta} H_{t}(s) \mathrm{d} s, \quad \int_{\alpha}^{\beta} \tilde{\tilde{H}}_{\tilde{\tau}}(s) \mathrm{d} s=\int_{2 c-\beta}^{2 c-\alpha} H_{t}(s) \mathrm{d} s \quad \text { for all } \alpha, \beta \in[c, b],
$$

where as before $\tilde{\tilde{H}}_{\tilde{\tau}}(s)$ and $\tilde{\tilde{H}}_{t}(s)$ denote $\tilde{H}_{\tilde{\tau}}$ and $\tilde{H}_{t}$ evaluated at $\left(s, \tilde{\tau}(s), \tilde{x}(s), \tilde{u}(s), \tilde{p}(s), 0, \tilde{\lambda}_{0}\right)$, and $H_{t}(s)$ is $H_{t}\left(s, x(s), u(s), p(s), 0, \lambda_{0}\right)$. More specifically, in the calculation (5.50)-(5.52), equations (5.51) and (5.52) should be modified to account for the $t$-dependence and (5.9) must be used instead of (5.5), as it is illustrated in the following

$$
\begin{aligned}
\tilde{\tilde{H}}(t) & \stackrel{(5.9)}{=} 2\left(\lambda_{0} K_{t_{1}}+\varphi_{t_{1}}^{T} \gamma\right)(a, b, x(a), x(b))+\int_{a}^{2 c-t} H_{t}(s) \mathrm{d} s+\int_{a}^{c} H_{t}(s) \mathrm{d} s+\int_{c}^{t} H_{t}(s) \mathrm{d} s \\
& \stackrel{(5.57)}{=} 2\left(\tilde{\lambda}_{0} K_{t_{1}}+\varphi_{t_{1}}^{T} \tilde{\gamma}_{2}\right)\left(\tilde{\tau}(b), b, \tilde{x}_{1}(b), \tilde{x}_{2}(b)\right)+\int_{t}^{b} \tilde{\tilde{H}}_{\tilde{\tau}}(s) \mathrm{d} s+\int_{c}^{b} \tilde{\tilde{H}}_{\tilde{\tau}}(s) \mathrm{d} s+\int_{c}^{t} \tilde{\tilde{H}}_{t}(s) \mathrm{d} s \\
& =2\left(\tilde{\lambda}_{0} K_{t_{1}}+\varphi_{t_{1}}^{T} \tilde{\gamma}_{2}\right)\left(\tilde{\tau}(b), b, \tilde{x}_{1}(b), \tilde{x}_{2}(b)\right)+2 \int_{c}^{b} \tilde{\tilde{H}}_{\tilde{\tau}}(s) \mathrm{d} s+\int_{c}^{t}\left(\tilde{\tilde{H}}_{t}(s)-\tilde{\tilde{H}}_{\tilde{\tau}}(s)\right) \mathrm{d} s .
\end{aligned}
$$


Hence, equation (5.46) is valid, and so is (5.45)(i). Similarly, using (5.10) instead of (5.9) we obtain (5.47), and also (5.45)(ii). For the "conversely" part of Proposition 5.6, equation $(5.56)$ is now $h_{t}(\tau, u(\tau))=H_{t}(\tau):=$ $H_{t}\left(\tau, x(\tau), u(\tau), p(\tau), 0, \lambda_{0}\right)$ and its proceeding calculation becomes

$$
\begin{aligned}
& H\left(t, x(t), u(t), p(t), 0, \lambda_{0}\right) \\
& \quad=\frac{1}{2} \tilde{H}\left(c, \tilde{\tau}(c), \tilde{x}(c), \tilde{u}(c), \tilde{p}(c), 0, \tilde{\lambda}_{0}\right)+\int_{c}^{t} H_{t}(\tau) \mathrm{d} \tau \\
& \stackrel{(5.46)}{=}\left(\tilde{\lambda}_{0} K_{t_{1}}+\varphi_{t_{1}}^{T} \tilde{\gamma}_{2}\right)\left(\tilde{\tau}(b), b, \tilde{x}_{1}(b), \tilde{x}_{2}(b)\right)+\int_{c}^{b} \tilde{\tilde{H}}_{\tilde{\tau}}(s) \mathrm{d} s+\int_{c}^{t} H_{t}(\tau) \mathrm{d} \tau \\
& \stackrel{(5.57)}{=}\left(\lambda_{0} K_{t_{1}}+\varphi_{t_{1}}^{T} \gamma\right)(a, b, x(a), x(b))+\int_{a}^{t} H_{t}(\tau) \mathrm{d} \tau
\end{aligned}
$$

that is, (5.9) holds. Using (5.47) in place of (5.46), the equality in (5.10) follows.

Acknowledgements. The authors wish to thank anonymous referees for valuable comments and suggestions regarding the subject of this paper, including those which eventually led to the inclusion of the results in Section 5.

\section{REFERENCES}

[1] J. Allwright and R. Vinter, Second order conditions for periodic optimal control problems. Control Cybernet. 34 (2005) 617-643.

[2] M.S. Aronna, J.F. Bonnans, A.V. Dmitruk and P.A. Lotito, Quadratic order conditions for bang-singular extremals. Numer. Algebra Control Optim. 2 (2012) 511-546.

[3] M. Bohner, Linear Hamiltonian difference systems: disconjugacy and Jacobi-type conditions. J. Math. Anal. Appl. 199 (1996) 804-826.

[4] V.G. Boltyanskii, Sufficient conditions for optimality and the justification of the dynamic programming method. SIAM J. Control Optim. 4 (1966) 326-361.

[5] J.F. Bonnans, X. Dupuis and L. Pfeiffer, Second-order necessary conditions in Pontryagin form for optimal control problems. SIAM J. Control Optim. 52 (2014) 3887-3916.

[6] L. Bourdin and E. Trélat, Pontryagin maximum principle for finite dimensional nonlinear optimal control problems on time scales. SIAM J. Control Optim. 51 (2013) 3781-3813.

[7] L. Bourdin and E. Trélat, Optimal sampled-data control, and generalizations on time scales. Math. Control. Relat. Fields 6 (2016) 53-94.

[8] H.I. Dwyer, Eigenvalues of Matrix Sturm-Liouville Problems with Separated or Coupled Boundary Conditions, Ph.D. dissertation, Northern Illinois University, DeKalb, IL (1993).

[9] H.I. Dwyer and A. Zettl, Computing eigenvalues of regular Sturm-Liouville problems. Electr. J. Differ. Equ. 1994 (1994) 10 pp.

[10] J.V. Elyseeva and R. Šimon Hilscher, Discrete oscillation theorems for symplectic eigenvalue problems with general boundary conditions depending nonlinearly on spectral parameter. Linear Algebra Appl. 558 (2018) 108-145.

[11] W.H. Fleming and R.W. Rishel, Deterministic and Stochastic Optimal Control, Applications of Mathematics, No. 1. SpringerVerlag, Berlin-New York (1975).

[12] R. Hilscher and V. Růžičková, Riccati inequality and other results for discrete symplectic systems. J. Math. Anal. Appl. 322 (2006) 1083-1098.

[13] R. Hilscher and V. Zeidan, Symplectic difference systems: variable stepsize discretization and discrete quadratic functionals. Linear Algebra Appl. 367 (2003) 67-104.

[14] R. Hilscher and V. Zeidan, Applications of time scale symplectic systems without normality. J. Math. Anal. Appl. 340 (2008) 451-465.

[15] R. Hilscher and V. Zeidan, Time scale embedding theorem and coercivity of quadratic functionals. Analysis (Munich) 28 (2008) $1-28$.

[16] R. Hilscher and V. Zeidan, Riccati equations for abnormal time scale quadratic functionals. J. Differ. Equ. 244 (2008) 1410-1447.

[17] R. Hilscher and V. Zeidan, Weak maximum principle and accessory problem for control problems on time scales. Nonlinear Anal. 70 (2009) 3209-3226.

[18] D.H. Jacobson, Extensions of Linear-Quadratic Controls, Optimization and Matrix Theory, Vol. 133 of Mathematics in Science and Engineering. Academic Press, London - New York - San Francisco (1977). 
[19] R.E. Kalman, The theory of optimal control and the calculus of variations. In Mathematical Optimization Techniques", Symposium on Mathematical Optimization Techniques, Santa Monica, CA, 1960, edited by R. Bellman. Univ. California Press, Berkeley, CA (1963) 309-331.

[20] W. Kratz, Quadratic Functionals in Variational Analysis and Control Theory, Vol. 6 of Mathematical Topics. Akademie Verlag, Berlin (1995).

[21] D.G. Luenberger, Linear and Nonlinear Programming, Addison-Wesley, Reading, MA (1984) 2nd edn.

[22] K. Malanowski and H. Maurer, Sensitivity analysis for parametric control problems with control-state constraints. Comput. Optim. Appl. 5 (1996) 253-283.

[23] H. Maurer, Sufficient conditions and sensitivity analysis for economic control problems. in "Optimal Control and Differential Games" (Vienna, 1997). Ann. Oper. Res. 88 (1999) 3-14.

[24] H. Maurer and H.J. Pesch, Solution differentiability for nonlinear parametric control problem. SIAM J. Control Optim. 32 (1994) 1542-1554.

[25] H. Maurer and H.J. Pesch, Solution differentiability for parametric nonlinear control problems with control-state constraints. J. Optim. Theory Appl. 86 (1995) 285-309.

[26] H. Maurer and S. Pickenhaim, Second-order sufficient conditions for control problems with mixed control-state constraints. $J$. Optim. Theory Appl. 86 (1995) 649-667.

[27] D. Orrell and V. Zeidan, Another Jacobi sufficiency criterion for optimal control with smooth constraints. J. Optim. Theory Appl. 58 (1988) 283-300.

[28] N.P. Osmolovskii, Sufficient quadratic conditions of extremum for discontinuous controls in optimal control problems with mixed constraints. J. Math. Sci. (N.Y.) 173 (2011) 1-106.

[29] L. Poggiolini and G. Stefani, Bang-singular-bang extremals: sufficient optimality conditions. J. Dyn. Control Syst. 17 (2011) 469-514.

[30] R. Šimon Hilscher, Eigenvalue theory for time scale symplectic systems depending nonlinearly on spectral parameter. Appl. Math. Comput. 219 (2012) 2839-2860.

[31] R. Šimon Hilscher and V. Zeidan, Hamilton-Jacobi theory over time scales and applications to linear-quadratic problems. Nonlinear Anal. 75 (2012) 932-950.

[32] R. Šimon Hilscher and V. Zeidan, Oscillation theorems and Rayleigh principle for linear Hamiltonian and symplectic systems with general boundary conditions. Appl. Math. Comput. 218 (2012) 8309-8328.

[33] R. Šimon Hilscher and V. Zeidan, Sufficiency and sensitivity for nonlinear optimal control problems on time scales via coercivity. ESAIM: COCV 24 (2018) 1705-1734.

[34] R. Šimon Hilscher and P. Zemánek, Weyl disks and square summable solutions for discrete symplectic systems with jointly varying endpoints. Adv. Differ. Equ. 2013 (2013) $232 .$.

[35] J.L. Speyer and D.H. Jacobson, Primer on Optimal Control Theory, Vol. 20 of Advances in Design and Control. Society for Industrial and Applied Mathematics (SIAM), Philadelphia, PA (2010).

[36] G. Stefani and P. Zezza, Constrained regular LQ-control problems. SIAM J. Control Optim. 35 (1997) 876-900.

[37] R. Vinter, Optimal Control, Systems \& Control: Foundations \& Applications, Birkhäuser, Boston, MA (2000).

[38] V. Zeidan, Sufficiency conditions with minimal regularity assumptions. Appl. Math. Optim. 20 (1989) $19-31$.

[39] V. Zeidan, The Riccati equation for optimal control problems with mixed state-control constraints: necessity and sufficiency. SIAM J. Control Optim. 32 (1994) 1297-1321.

[40] V. Zeidan, New second-order optimality conditions for variational problems with $C^{2}$-Hamiltonians. SIAM J. Control Optim. 40 (2001) 577-609.

[41] V. Zeidan and P. Zezza, Normality for the problem of Bolza with an inequality state constraint. Geoffrey J. Butler Memorial Conference in Differential Equations and Mathematical Biology (Edmonton, AB, 1988). Rocky Mountain J. Math. 20 (1990) $1235-1248$ 